\title{
Mathematical study of scattering resonances
}

\author{
Maciej Zworski ${ }^{1}$
}

Received: 9 September 2016 / Accepted: 16 February 2017 / Published online: 8 March 2017

(C) The Author(s) 2017. This article is published with open access at Springerlink.com

\begin{abstract}
We provide an introduction to mathematical theory of scattering resonances and survey some recent results.
\end{abstract}

\section{Contents}

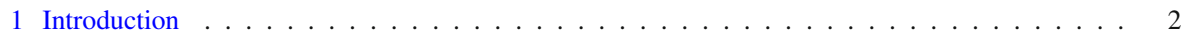

1.1 Motivation from quantum mechanics . . . . . . . . . . . . . . . . . . . . . . . . . . . . . . . . . . . . . . . . . .

1.2 Scattering of waves in one dimension . . . . . . . . . . . . . . . . . . . . . . . . . . . . . . . . .

1.3 Resonances in the semiclassical limit . . . . . . . . . . . . . . . . . . . . 8

1.4 Other examples from physics and engineering . . . . . . . . . . . . . . . . . 10

2 Potential scattering in three dimensions . . . . . . . . . . . . . . . . . . 11

2.1 The free resolvent . . . . . . . . . . . . . . . . . . . . . . . . 12

2.2 Meromorphic continuation and definition of resonances . . . . . . . . . . . . . . . 15

2.3 Upper bound on the number of resonances . . . . . . . . . . . . . . . . . . . . . 18

2.4 Resonance free regions . . . . . . . . . . . . . . . . . . . . . . . . . 22

2.5 Resonance expansions of scattered waves . . . . . . . . . . . . . . . . . . . . 26

2.6 Complex scaling in dimension one . . . . . . . . . . . . . . . . . . . . . . . . . . . . . . . . . . . . . . . . . 31

2.7 Other results and open problems . . . . . . . . . . . . . . . . . . 35

3 Some recent developments . . . . . . . . . . . . . . . . . . . . . . 36

3.1 Meromorphic continuation in geometric scattering . . . . . . . . . . . . . . . . . . . 37

3.2 Resonance free regions . . . . . . . . . . . . . . . . . . . . . . . . . 43

3.3 Resonance expansions in general relativity . . . . . . . . . . . . . . . . . . . . . 53

3.4 Upper bounds on the number of resonances: fractal Weyl laws . . . . . . . . . . . . . . . 56

4 Pollicott-Ruelle resonances from a scattering theory viewpoint . . . . . . . . . . . . . . . 61

4.1 Anosov dynamical systems . . . . . . . . . . . . . . . . . . . . . 62

Communicated by Ari Laptev.

$凶 \quad$ Maciej Zworski

zworski@math.berkeley.edu

1 Department of Mathematics, University of California, Berkeley, CA 94720, USA 
4.2 Definition of Pollicott-Ruelle resonances . . . . . . . . . . . . . . . . . . . . . . . 64

4.3 Connections to dynamical zeta functions . . . . . . . . . . . . . . . . . . . . . 69

4.4 Distribution of Pollicott-Ruelle resonances . . . . . . . . . . . . . . . . . . . . . 73

References.............................. 76

\section{Introduction}

Scattering resonances appear in many branches of mathematics, physics and engineering. They generalize eigenvalues or bound states to systems in which energy can scatter to infinity. A typical state has then a rate of oscillation (just as a bound state does) and a rate of decay. Although this notion is intrinsically dynamical, an elegant mathematical formulation comes from considering meromorphic continuations of Green's functions or scattering matrices. The poles of these meromorphic continuations capture the physical information by identifying the rate of oscillations with the real part of a pole and the rate of decay with its imaginary part. The resonant state, which is the corresponding wave function, then appears in the residue of the meromorphically continued operator. An example from pure mathematics is given by the zeros of the Riemann zeta function: they are the resonances of the Laplacian on the modular surface. The Riemann hypothesis then states that the decay rates for the modular surface are all either 0 or $\frac{1}{4}$. A standard example from physics is given by shape resonances created when the interaction region is separated from free space by a potential barrier. The decay rate is then exponentially small in a way depending on the width of the barrier.

In this article we survey some foundational and some recent aspects of the subject selected using the perspective and experience of the author. Proofs of many results can be found in the monograph written in collaboration with Semyon Dyatlov [80] to which we provide frequent references.

What we call scattering resonances appear under different names in different fields: in quantum scattering theory they are called quantum resonances or resonance poles. In obstacle scattering, or more generally wave scattering, they go by scattering poles. In general relativity the corresponding complex modes of gravitational waves are known as quasi-normal modes. The closely related poles of power spectra of correlations in chaotic dynamics are called Pollicott-Ruelle resonances. We will discuss mathematical results related to each of these settings stressing unifying features.

The survey is organized as follows:

- in the introduction we provide a basic physical motivation from quantum mechanics, discuss the case of the wave equation in one dimension, intuitions behind semiclassical study of resonances and some examples from modern science and engineering;

- in Sect. 2 we present scattering by bounded compactly supported potentials in three dimensions and prove meromorphic continuation of the resolvent (Green function), an upper bound on the counting function, existence of resonance free regions and expansions of waves; we also explain the method of complex scaling in the elementary setting of one dimension; finally we discuss recent progress and open problems in potential scattering; 
- we devote Sect. 3 to a survey of recent results organized around topics introduced in the special setting of Sect. 2: meromorphic continuation for asymptotically hyperbolic spaces, fractal upper bounds in physical and geometric settings, resonance free strips in chaotic scattering and resonance expansions; we also provide some references to recent progress in some of the topics not covered in this survey;

- Section 4 surveys the use of microlocal/scattering theory methods in the study of chaotic dynamical systems. Their introduction by Faure-Sjöstrand [84] and Tsujii [261] led to rapid progress which included a microlocal proof of Smale's conjecture about dynamical zeta functions [78], first proved shortly before by Giulietti-Liverani-Pollicott [109]. We review this and other results, again related to upper bounds, resonance free strips and resonances expansions.

\subsection{Motivation from quantum mechanics}

In quantum mechanics a particle is described by a wave function $\psi$ which is normalized in $L^{2},\|\psi\|_{L^{2}}=1$. The probability of finding the particle in a region $\Omega$ is the given by the integral of $|\psi(x)|^{2}$ over $\Omega$. A pure state is typically an eigenstate of a Hamiltonian $P$ and hence the evolved state is given by $\psi(t):=e^{-i t P} \psi=e^{-i t E_{0}} \psi$ where $P \psi=$ $E_{0} \psi$. In particular the probability density does not change when the state is propagated.

An example could be given by the Bloch electron in a molecular corral shown in Fig. 1. However the same figure also shows that the measured states have non-zero "widths" - the peak is not a delta function at $E_{0}$ - and hence can be more accurately modeled by resonant states. Scattering or quantum resonances are given as complex numbers $E_{0}-i \Gamma / 2$ and the following standard argument of the physics literature explains the meaning of the real and imaginary parts: a time dependent pure resonant state propagates according to $\psi(t)=e^{-i t E_{0}-t \Gamma / 2} \psi(0)$ so that the probability of survival beyond time $t$ is $p(t)=|\psi(t)|^{2} /|\psi(0)|^{2}=e^{-\Gamma t}$. This explains why the convention for the imaginary part of a resonance is $\Gamma / 2$. Here we neglected the issue that $\psi(0) \notin L^{2}$ which is remedied by taking the probabilities over a bounded interaction region. In the energy representation the wave function is given

$$
\varphi(E):=\mathcal{F}^{-1} \psi(E)=\frac{1}{\sqrt{2 \pi}} \int_{0}^{\infty} e^{-i t E_{0}-t \Gamma / 2} \psi(0) d t=\frac{1}{\sqrt{2 \pi} i} \frac{\psi(0)}{E_{0}-i \Gamma / 2-E},
$$

which means that the probability density of the resonance with energy E is proportional to the square of the absolute value of the right hand side. Consequently the probability density is

$$
\frac{1}{2 \pi} \frac{\Gamma}{\left(E-E_{0}\right)^{2}+(\Gamma / 2)^{2}} d E,
$$

and this Lorentzian is the famous Breit-Wigner distribution. This derivation is of course non-rigorous and there are many mathematical issues:

- the state $\psi(0)$ is not in $L^{2}$ - it has physical meaning only in the "interaction region"; that is justified differently in Euclidean and non-Euclidean scatteringsee Sects. 2.2, 2.6 and 3.1 respectively.

- it is not clear why the evolution of a physical state should have an expansion in terms of resonant states; that is justified using meromorphic continuation and asymptotic control of Green's function and-see Sect. 2.5; 


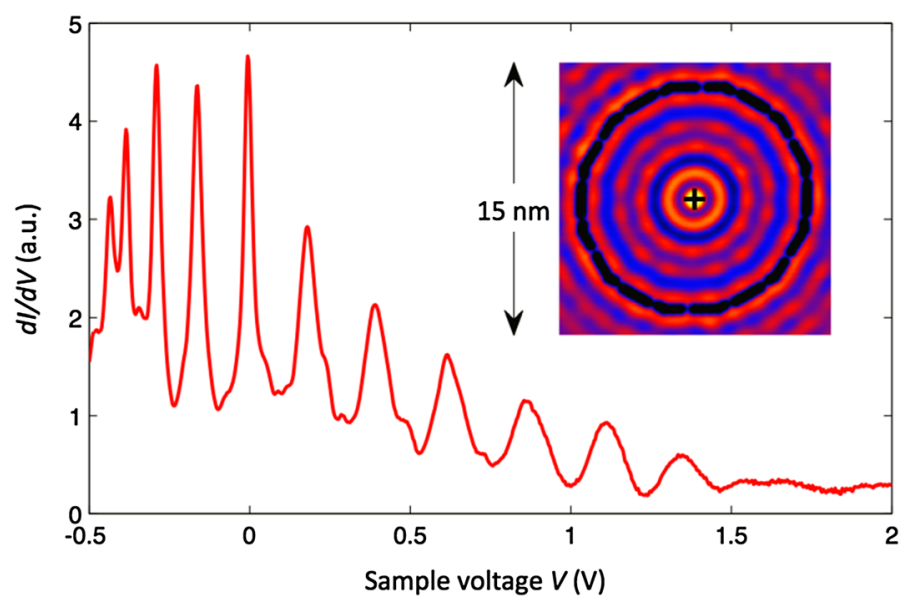

Fig. 1 A scanning tunneling microscope (STM) spectrum is a plot of $d I / d V$ ( $I$ being the current) as a function of bias voltage $V$. According to the basic theory of STM, this reflects the sample density of states as a function of energy with respect to the Fermi energy (at $V=0$ ). This spectrum shows the series of surface state electron resonances in inside a circular quantum corral on $\mathrm{Cu}(111)$ —see Fig. 3 for a visualization of the relation between the peaks and the complex resonance poles. The bulk bands contribute to a gradually varying background in this spectrum. The setpoint was $V_{0}=1 \mathrm{~V}$ and $I_{0}=10 \mathrm{nA}$ and the modulation voltage was $V_{\mathrm{rms}}=4 \mathrm{mV}$. Inset: a low-bias topograph of the corral studied $\left(17 \times 17 \mathrm{~nm}^{2}, V=10 \mathrm{mV}\right.$, $I=1 \mathrm{nA}$ ). The corral is made from $84 \mathrm{CO}$ molecules adsorbed to $\mathrm{Cu}(111)$ and has an average radius of $69.28 \AA$ - the date and images are from the Manoharan Lab at Stanford [186]. The large amplitude in the center of the top graph is a reflection of the sharp peak seen in the spectrum at $V=0$. For another quantum corall, in the shape of the Bunimovich stadium and made out of iron atoms, see Fig. 14

- one needs to justify the passage from the Fourier transform $t \mapsto E$ to the probability distribution (1.1); that is done using the scattering matrix or spectral measure - see the end of Sect. 2.5 .

Returning to physical motivation, one should stress that in practice there are many deviations from the simple formula (1.1), especially at high energies and in the presence of overlapping resonances. In Fig. 1 we see clear Lorentzian peaks and individual resonances can be recovered. In the experiment shown in Fig. 2 the resonances overlap and the peaks in scattering data do not have the simple interpretation using (1.1). The density laws (Weyl laws) for counting of resonant states are more complicated and richer as they involve both energy and rates of decay. Even the leading term can be affected by dynamical properties of the system-see Sects. 2.3 and 3.4.

A more abstract version of Fig. 1 is shown in Fig. 3 where correlations of evolved states and unevolved states are shown with peaks corresponding to poles of their Fourier transforms (power spectra).

\subsection{Scattering of waves in one dimension}

A simple mathematical example is given by scattering by compactly supported potentials in dimension one, see Fig. 4 for an example of such potential. Scattering 

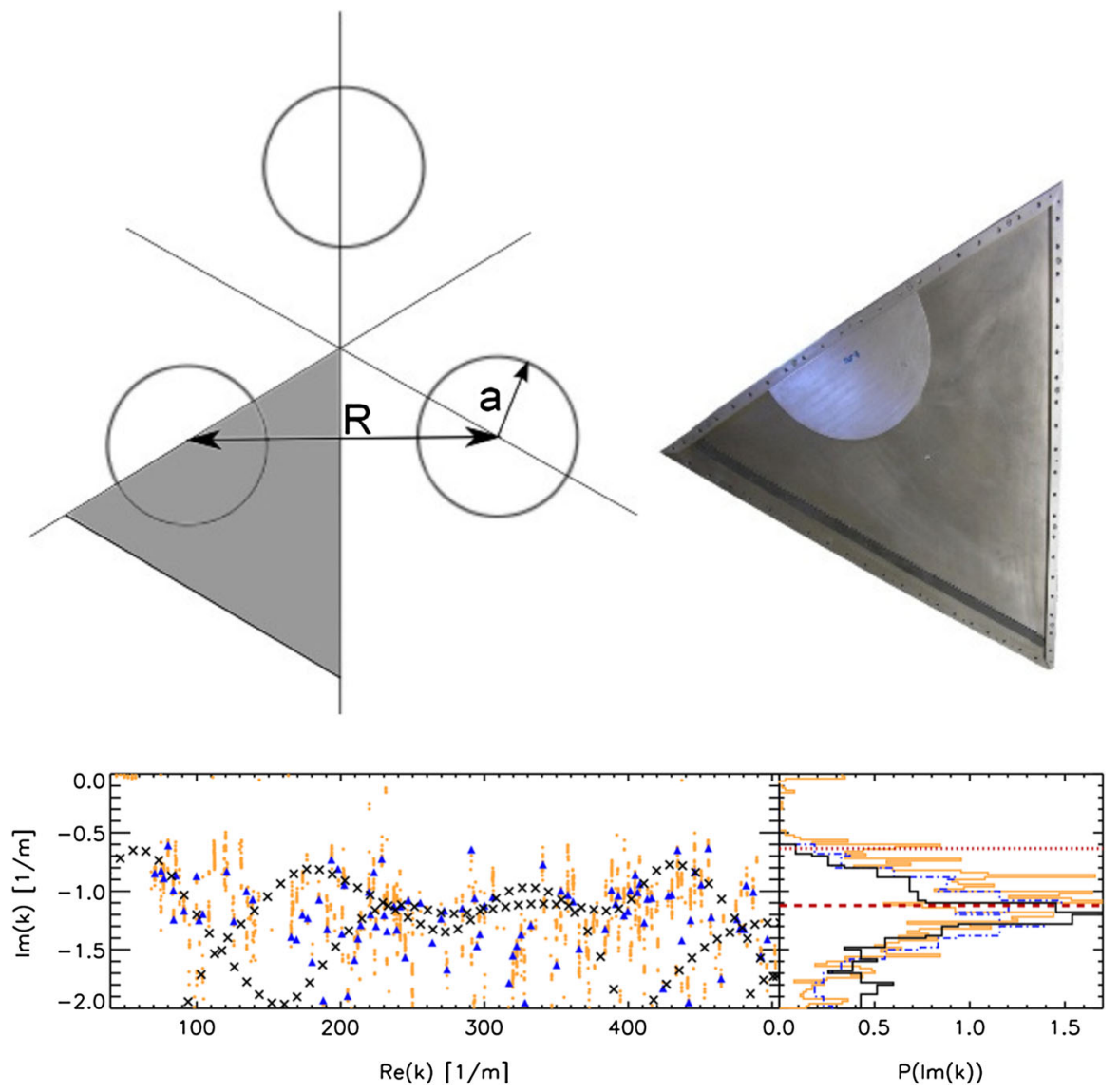

Fig. 2 The experimental set-up for the study of the fractal Weyl law [216] and of the distribution of resonance widths [13]. On the left panel below the resonances for the aspect ration 5.5 in the complex $k$-plane are shown as well as the distribution of the imaginary parts of $k$ in the right panel. The orange clouds correspond to all resonances resulting in a good reconstruction as well as the orange histogram. Note that many orange dots might overlay each other. Blue triangles and blue dashed-dotted histogram describe the set belonging to the best reconstruction. Black crosses are the numerically calculated poles and the solid black histogram the corresponding distribution. The dotted red line in the right panel is $P(1 / 2)$, the red dashed line half of the classical decay rate $P(1) / 2$. Here $P(s)$ is the topological pressure associated to the unstable Jacobian (colour figure online)

resonances are the rates of oscillations and decay of the solution of the wave equation and Fig. 6 shows such a solution.

We see the main wave escape and some trapped waves bounce in the well created by the potential and leak out. Figure 7 shows the values of the solution at one point. Roughly speaking if $u(t, x)$ is a solution of the wave equation $\left(\partial_{t}^{2}-\partial_{x}^{2}+V(x)\right) u=0$ with localized initial data then

$$
u(t, x) \sim \sum_{\operatorname{Im} \lambda_{j}>-A} e^{-i \lambda_{j} t} u_{j}(x)+\mathcal{O}_{K}\left(e^{-t A}\right), \quad x \in K \Subset \mathbb{R},
$$




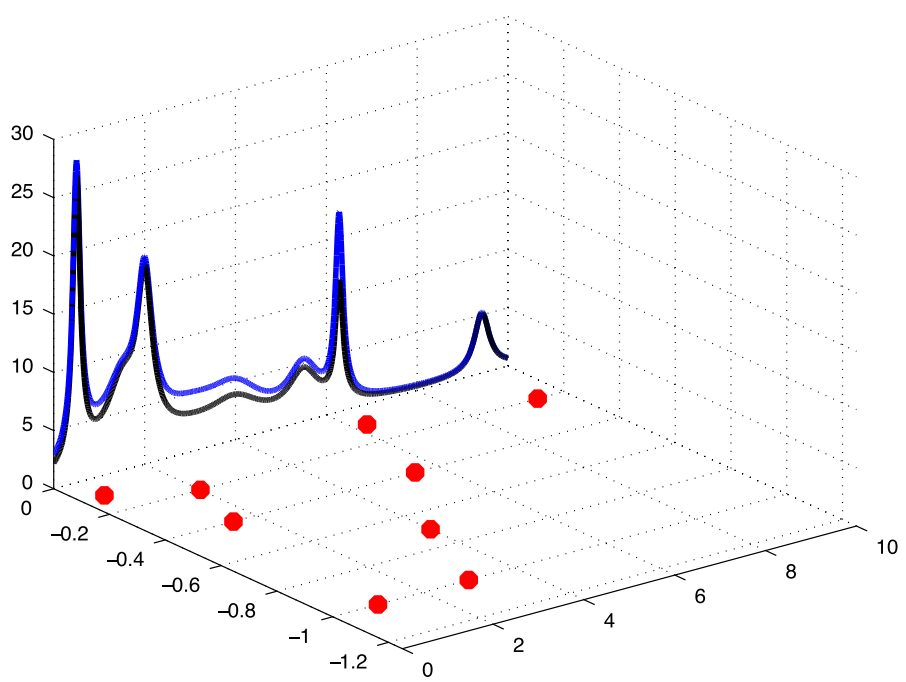

Fig. 3 If $U(t)$ is a propagator and $f, g$ are states then the correlation (the evolution of $f$ measured by $g$ ) is given by $\rho_{f, g}(t)=\langle U(t) f, g\rangle$. For instance the evolution could come from a flow $\varphi_{t}: M \rightarrow M$ on a compact manifold, $U(t) f(x)=f\left(\varphi_{-t}(x)\right)$. The power spectrum of correlations is given by $\widehat{\rho}_{f, g}(\lambda):=$ $\int_{0}^{\infty} \rho_{f, g}(t) e^{i \lambda t} d t$. Resonances are the poles of $\widehat{\rho}_{f, g}(\lambda)$ and are independent of $f$ and $g$. The figure shows a schematic correspondence between the power spectrum for different states and these poles: the real part corresponds to the location of a peak in the power spectrum and the imaginary to its width; the $x$ axis is the real part (frequency $\lambda$ ), $y$ axis the imaginary (rate of decay), $z$ axis $\left|\hat{\rho}_{f, g}(\lambda)\right|$. Unlike the power spectrum which depends on $f$ and $g$, the poles depend only on the system

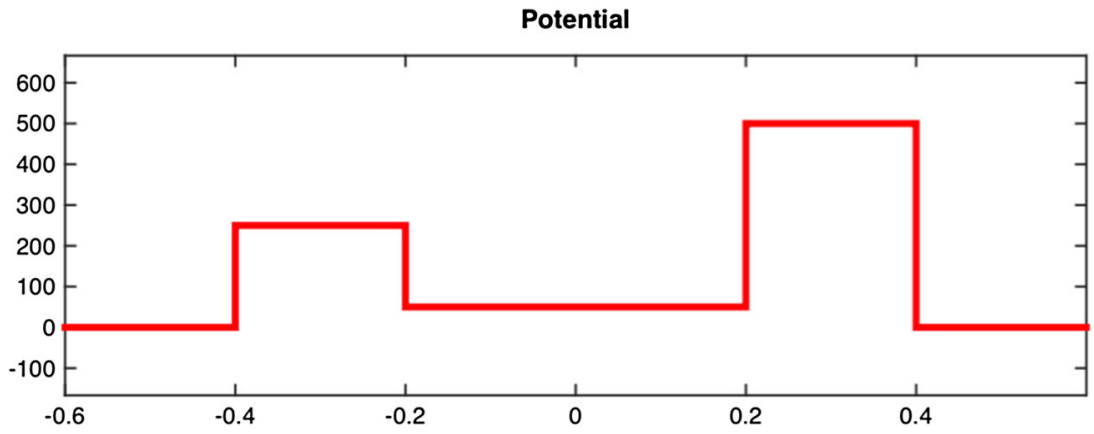

Fig. 4 A simple one dimensional potential with resonances shown in Fig. 5. Same potential is used to see propagation, trapping and tunneling of a wave in Fig. 6

where $\lambda_{j}$ are complex numbers with $\operatorname{Im} \lambda_{j}<0$. They are independent of the initial data and are precisely the scattering resonances-see Sect. 2.5 for the precise statement. The expansion (1.2) is formally related to the Breit-Wigner distribution (1.1) via the Fourier transform-see Fig. 3.

Harmonic inversion methods, the first being the celebrated Prony algorithm [217], can then be used to extract scattering resonances from solutions of the wave equation (as shown in Fig. 7; see for instance [171]). The resulting complex numbers, that is the resonances for the potential in Fig. 4 are shown in Fig. 5. 


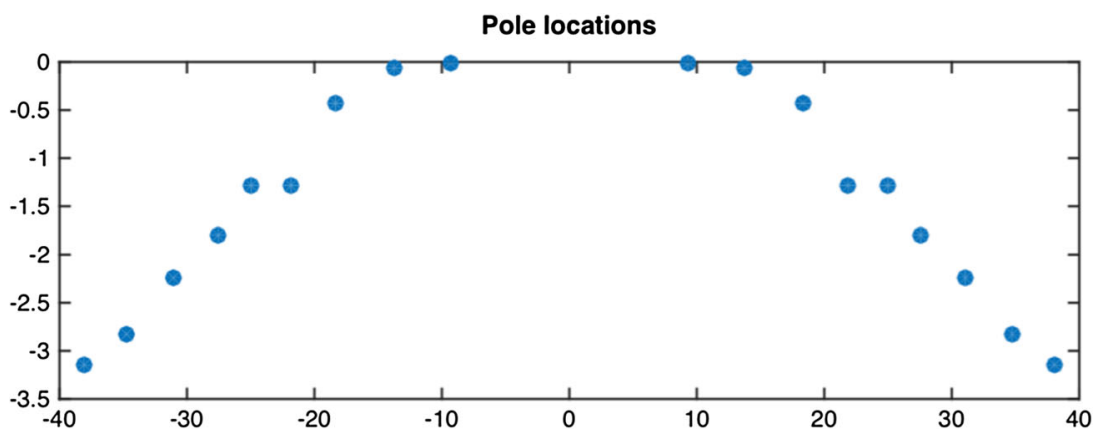

Fig. 5 Scattering poles of the potential shown in Fig. 4. They are computed very accurately using a Matlab code [21]

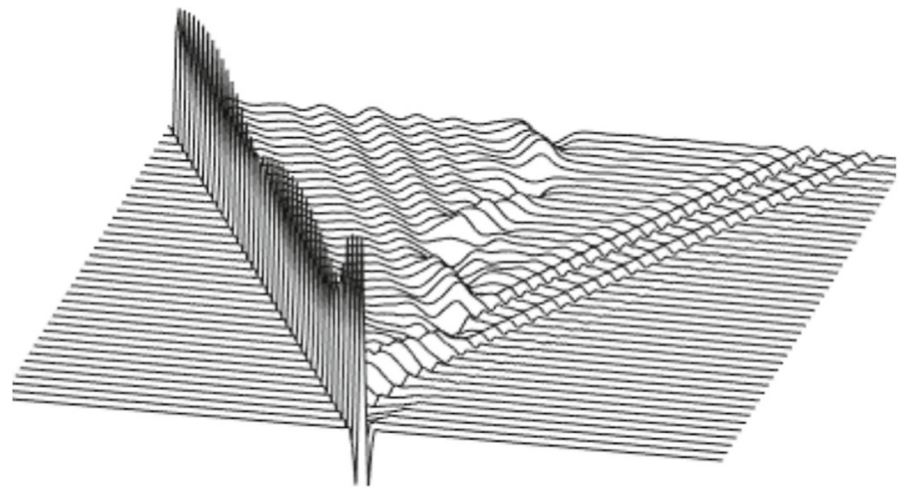

Fig. 6 A solution of the wave equation $\partial_{t}^{2} u-\partial_{x}^{2} u+V u=0$ where $V$ is shown in Fig. 4. The initial data is localized near 0 and the time variable $t$ points away from the viewer

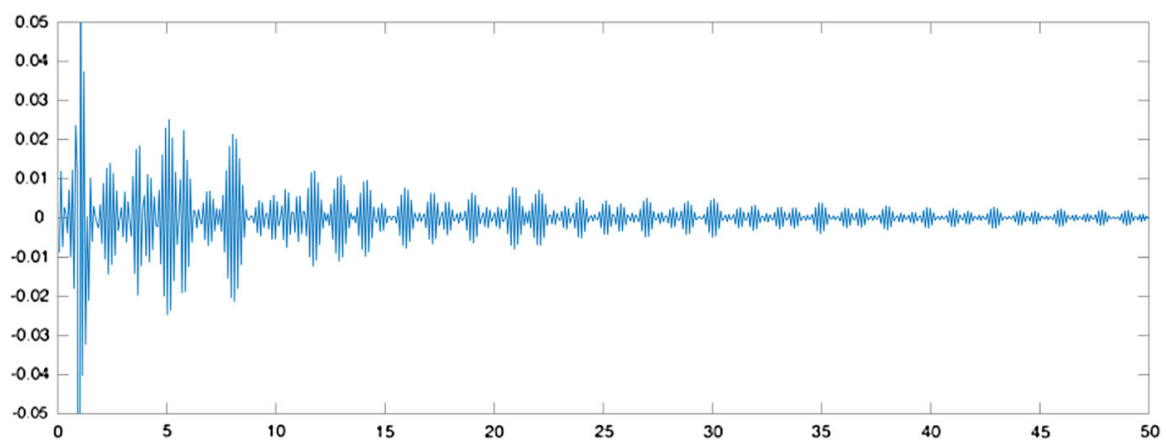

Fig. 7 The plot of $u(0, t)$ showing oscillations and decay of the solution from Fig. 6 in the interaction region. The principal decay rate of the wave is determined by the resonance(s) closest to the real axis. For that we need to justify the expansion (1.2)—see Theorem 5 


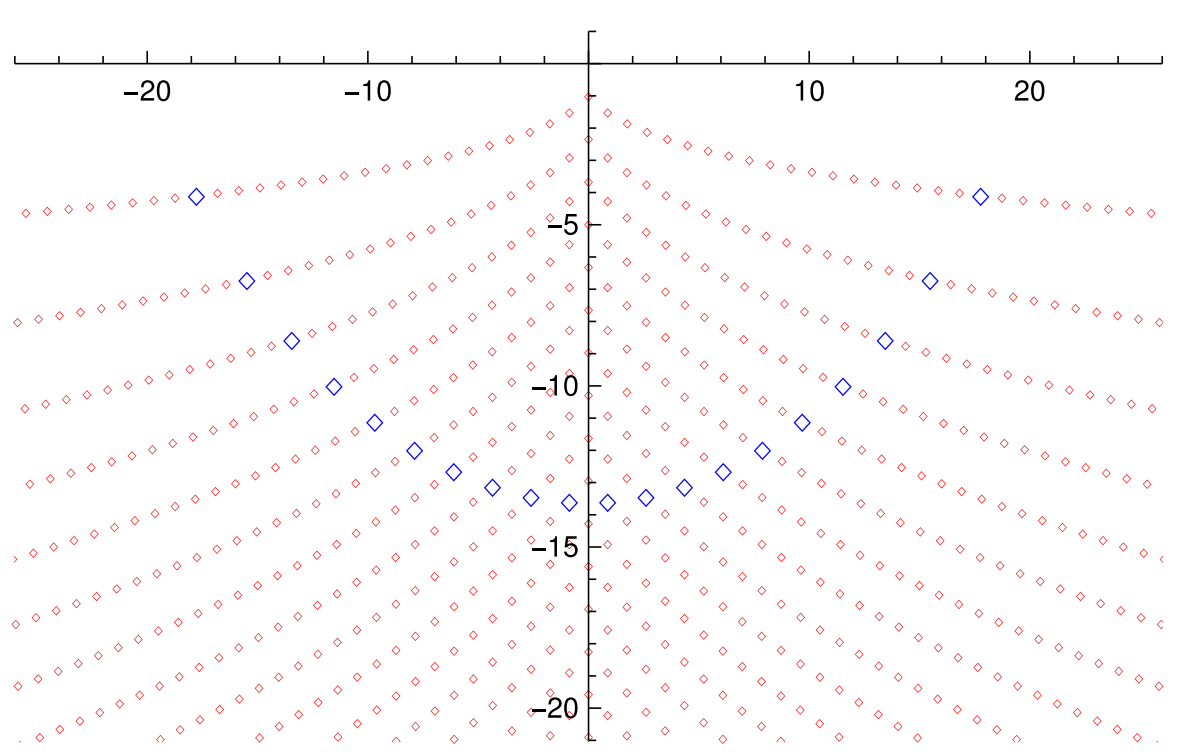

Fig. 8 Resonances for the sphere in three dimensions. For each spherical momentum $\ell$ they are given by solutions of $H_{\ell+\frac{1}{2}}^{(2)}(\lambda)=0$ where $H_{v}^{(2)}$ is the Hankel function of the second kind and order $v$. Each zero appears as a resonance of multiplicity $2 \ell+1$ : the resonances with $\ell=20$ are highlighted

\subsection{Resonances in the semiclassical limit}

For some very special systems resonances can be computed explicitely. One famous example is the Eckart barrier: $-\partial_{x}^{2}+\cosh ^{-2} x$. It falls into the general class of PöschelTeller potentials which can also be used to compute resonances of hyperbolic spaces or hyperbolic cylinders - see [31]. Another example is given by scattering in the exterior of a spherical obstacle. In this case scattering resonances are zeros of Hankel functions which in odd dimensions are in fact zeros of explicit polynomials—see [255] and Fig. 8.

In general however it is impossible to obtain an explicit description of individual resonances. Hence we need to consider their properties and their distribution in asymptotic regimes. For instance in the case of obstacle scattering that could mean the high energy limit. In the case of the sphere in Fig. 8 that corresponds to letting the angular momentum $\ell \rightarrow+\infty$. For a general obstacle that means considering resonances as $|\lambda| \rightarrow+\infty$ and $|\operatorname{Im} \lambda| \ll|\lambda|$.

The high energy limit is an example of a semiclassical limit. To describe it we consider resonances of the Dirichlet realization of $-h^{2} \Delta+V$ on $\mathbb{R}^{n} \backslash \mathcal{O}$ in bounded subsets of $\mathbb{C}$ as $h \rightarrow 0$. When $V \equiv 0$ that corresponds to the high energy limit for obstacle problems and when $\mathcal{O}=\emptyset$ to Schrödinger operators.

In the case of semiclassical Schrödinger operators, the properties of the classical energy surface $\xi^{2}+V(x)=E$ can be used to study resonances close to $E \in \mathbb{R}$. Figure 9 shows some of the principles in dimension one. The classical energy surfaces are in this case integral curves of the Hamilton flow, $\dot{x}=2 \xi, \dot{\xi}=-V^{\prime}(x)$ (Newton equations) and the properties of this flow determine location of resonances when $h$ 


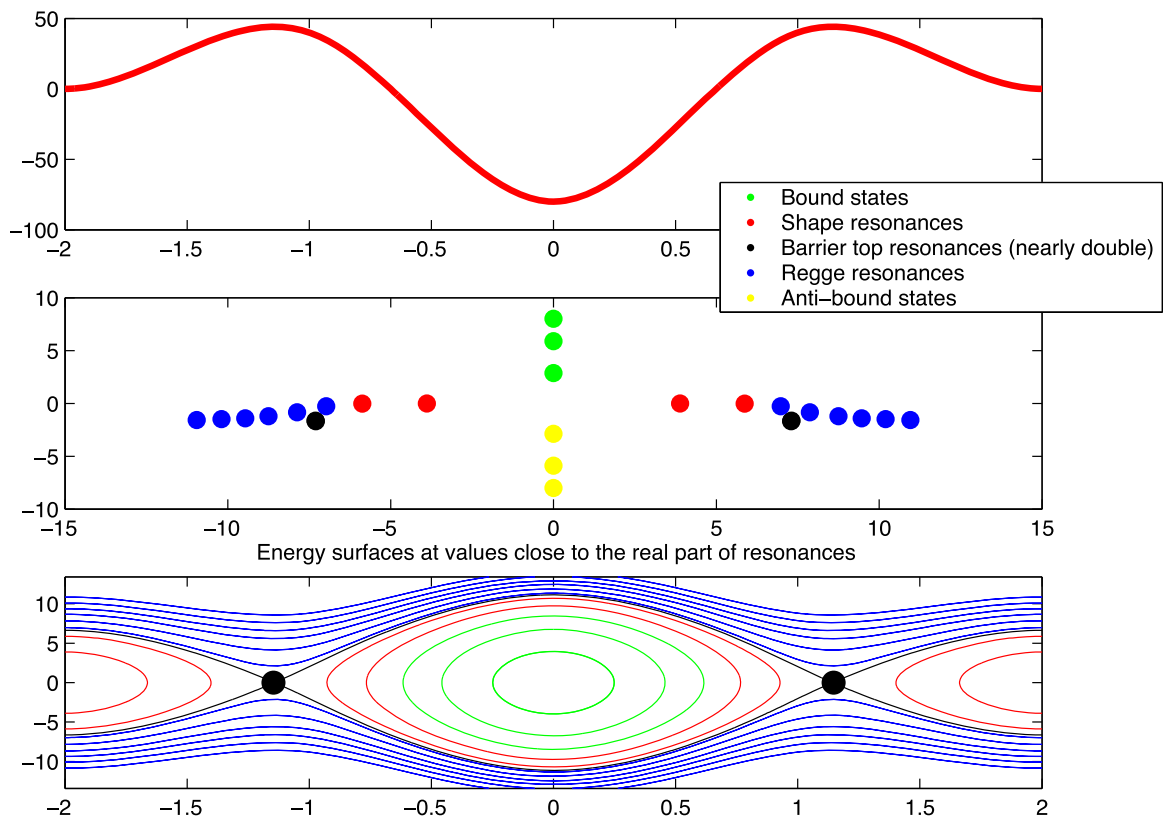

Fig. 9 Resonances corresponding to different dynamical phenomena as poles of continuation of the resolvent $\lambda \rightarrow\left(-h^{2} \partial_{x}^{2}+V(x)-\lambda^{2}\right)^{-1}$ from $\operatorname{Im} \lambda>0$ to $\operatorname{Im} \lambda \leq 0$-see Sect. 2.2. The bound states are generated by negative level sets of $\xi^{2}+V(x)$ satisfying Bohr-Sommerfeld quantization conditions. Bounded positive level sets of $\xi^{2}+V(x)$ can also satisfy the quantization conditions but they cannot produce bound state- tunnelling to the unbounded components of these level sets is responsible for resonances with exponentially small $\left(\sim e^{-S / h}\right)$ imaginary parts/width. The unstable trapped points corresponding to maxima of the potential produce resonances which are at distance $h$ of the real axis

is small. The level sets which are totally trapped and satisfy quantization conditions correspond to bound states. The level sets which have a trapped component but also an unbounded component, give rise to resonances with exponentially small decay rates, $e^{-S / h}$. That make sense since the corresponding quantum state take an exponentially long time to tunnel through the barrier (see [125] for a general theory and [95,114] for some recent results and references). Then we have energy levels which contain fixed points: that is an example of normally hyperbolic trapping which produces resonances at distance $h$ from the real axis-see Sect. 3.2 for a general discussion and [220] for precise analysis in one dimension and more references. Interestingly the same form of trapping occurs in the setting of rotating black holes, see Sect. 2.5 and [76]. Finally the unbounded trajectories would not produce resonances close (that is with imaginary parts tending to 0 with $h \rightarrow 0$ ) to the real axis if our potential were real analytic. But in the compactly supported, non smooth, case the singularities at the boundary of the support produce resonances at distance $M h \log \frac{1}{h}$ where $M$ depends on the regularity of the potential at the boundary of the support. (We call them Regge resonances as they were investigated in [221] but should not be confused with closely related Regge poles [113].) The anti-bound states are too deep in the lower half plane to have dynamical interpretation. They seem to be almost symmetric to bound states and in fact when the 

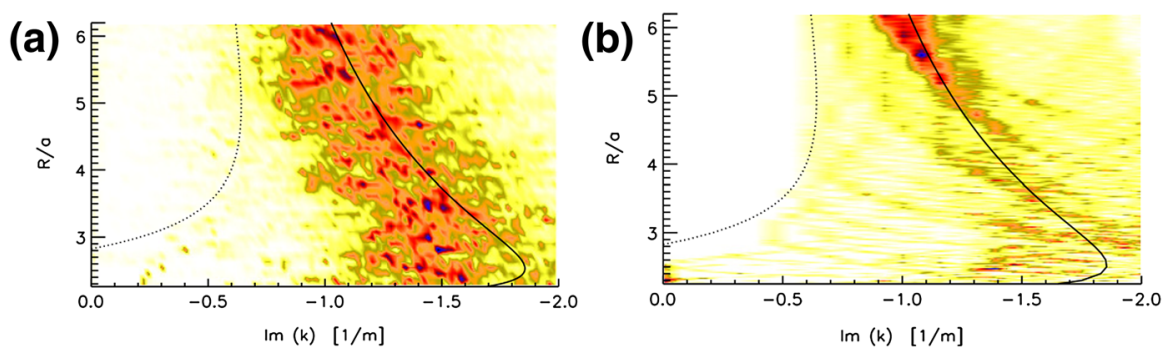

Fig. 10 Distribution of resonance width (decay rates) for a three disc scatterer [13] in a symmetry reduced microwave model shown in Fig. 2: the plots show the density of values of resonance width, $\operatorname{Im} k$, for a range of rest frequencies $\left(40 \mathrm{~m}^{-1} \leq \operatorname{Re} k \leq 500 \mathrm{~m}^{-1}\right.$ ) against the aspect ration of a three disc system (see Fig. 2). The experimental data (left) and numerical data (right) are compared. The dotted line shows the topological pressure at $\frac{1}{2}$ and the solid line $\frac{1}{2}$ of the classical decay rate as functions of the aspect ratio. The former predicts the resonance free region and the latter, the concentration of decay rates

potential is positive near the boundary of its support they are exponentially close to the reflection of bound states [72].

\subsection{Other examples from physics and engineering}

We present here a few recent examples of scattering resonances appearing in physical systems.

Figure 1 shows resonance peaks for a scanning tunneling microscope experiment where a circular quantum corral of CO molecules is constructed-see [187] and references given there. The resonances are very close to eigenvalues of the Dirichlet Laplacian (rescaled by $\hbar^{2} / m_{\text {eff }}$ where $m_{\text {eff }}$ is the effective mass of the Bloch electron). Mathematical results explaining existence of resonances created by a barrier (here formed by a corral of CO molecules) can be found in [194].

Figure 2 shows an experimental set-up for microwave cavities used to study scattering resonances for chaotic systems. Density of resonance was investigated in this setting in [216] and that is related to semiclassical upper bounds in Sect. 3.4. In [13] dependence of resonance free strips on dynamical quantities was confirmed experimentally and Sect. 3.2 contains related mathematical results and references. The experimental and numerical findings in [13] are presented in Fig. 10.

Figure 11 shows a MEMS (the acronym for the microelectromechanical systems) resonator. The numerical calculations [20] in that case are based on the complex scaling technique, presented in a model case in Sect. 2.6, adapted to the finite element methods. In that field it is known as the method of perfectly matched layers [19].

Figure 12 shows the profile of gravitational waves recently detected by the Laser Interferometer Gravitational-Wave Observatory (LIGO) and originating from a binary black hole merger. Resonances for such waves are known by the name of quasi-normal modes in physics literature and are the characteristic frequencies of the waves emitted during the ringdown phase of the merger, when the resulting single black hole settles down to its stationary state - see for instance [66,76,157] and Sect. 3.3.

Our last example is a proposal of using Pollicott-Ruelle resonances in climate study by Chekroun et al [44]. These are the resonances appearing in expansions of 


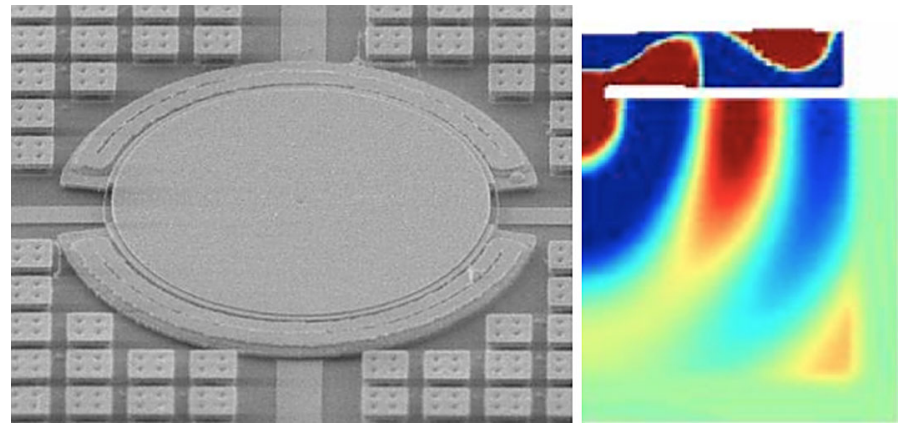

Fig. 11 A MEMS device on the left has resonances investigated using the complex scaling/perfectly matched layer methods [20]—see Sect. 2.6. A numerically constructed resonant state for the elasticity operator used to model the system — see Definition 2 for a simpler case-is shown on the right

Livingston, Louisiana (L1)
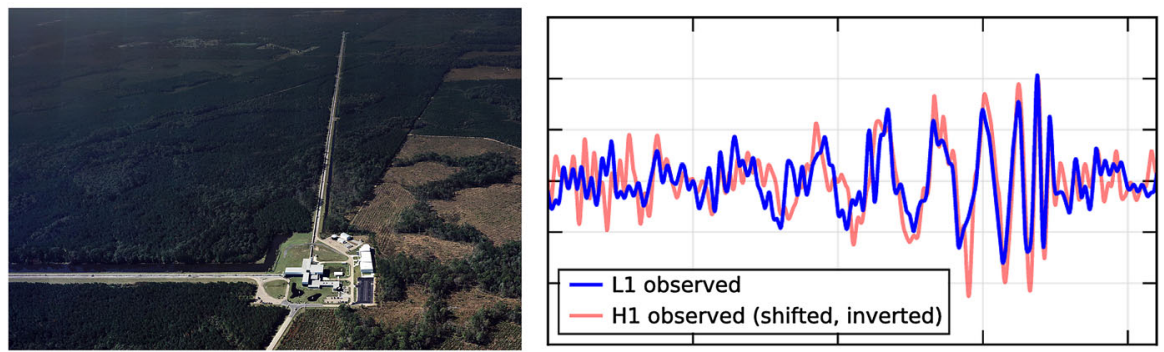

Fig. 12 Left an aerial view of the LIGO laboratory in Livingston, Louisiana, US. Right: the gravitational wave signal observed on September 14, 2015 simultaneously by LIGO Livingston (blue) and LIGO Hanford (red); see [1]. Quasi-normal modes, as scattering resonances are called in the context of gravitational waves, are supposed to appear in the "ringdown phase" but have not been observed yet. The picture is obtained from the LIGO Open Science Center, https://losc.ligo.org, a service of LIGO Laboratory and the LIGO Scientific Collaboration. LIGO is funded by the U.S. National Science Foundation (colour figure online)

correlations of chaotic flows and for the new mathematical developments in their study see Sect. 4. The idea in [44] is to use these resonances to encode information about low-frequency variability of turbulent flows in the atmosphere and oceans. The spectral gap-defined as the distance between the Ruelle-Pollicott resonances and the unitarity axis - is used to see the roughness of parameter dependences in different models-see Fig. 13. The authors claim that "links between model sensitivity and the decay of correlation properties are not limited to this particular model and could hold much more generally".

\section{Potential scattering in three dimensions}

Operators of the form $P_{V}:=-\Delta+V$, where $\Delta=\partial_{x_{1}}^{2}+\partial_{x_{2}}^{2}+\partial_{x_{3}}^{2}$, and where $V$ is bounded and compactly supported, provide a setting in which one can easily present basic theory. Despite the elementary set up, interesting open problems remain-see Sect. 2.7. In this section we prove meromorphic continuation of the resolvent of $P_{V}$, 

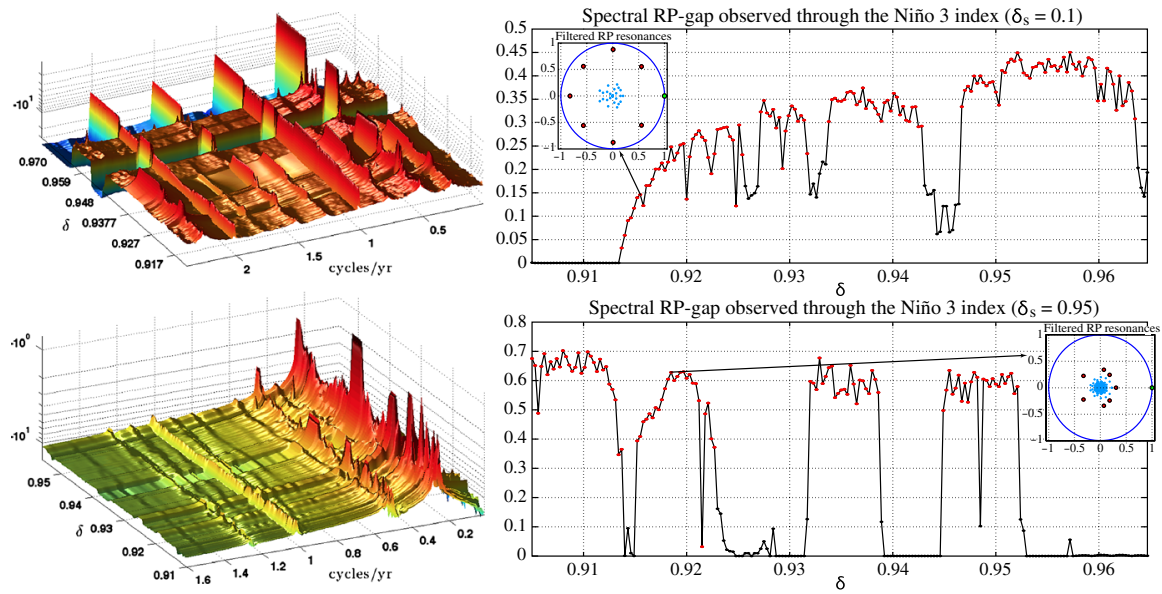

Fig. 13 Left parameter (denoted by $\delta$ ) dependence of power spectra of El Niño models considered in [44]. Right the dependence of the spectral gap on the same parameter $\delta$; here the resonance of maps obtained by using Markov partitions are presented and they lie inside of the unit disk. The resonance for flows are obtained by taking logarithms which is similar to the case of resonances of quantum maps-see Fig. 23

$R_{V}(\lambda):=\left(P_{V}-\lambda^{2}\right)^{-1}$, give a sharp bound on the number of resonances in discs, show existence of resonance free regions and justify the expansion (1.2). We also explain the method of complex scaling in the simplest setting of one dimension. These are the themes which reappear in Sects. 3, 4 when we discuss some recent advances. However, in the setting of this section we can present them with complete proofs. In Sect. 2.4 we also review some results in obstacle scattering as they fit naturally in our narrative.

\subsection{The free resolvent}

Let $P_{0}:=-\Delta$. This is a self-adjoint unbounded operator on $L^{2}\left(\mathbb{R}^{3}\right)$. We consider its resolvent, writing the spectral parameter as $\lambda^{2}$. For $\operatorname{Im} \lambda>0$,

$$
R_{0}(\lambda) f(x)=\int_{\mathbb{R}^{3}} R_{0}(\lambda, x, y) f(y) d y, \quad f \in L^{2}\left(\mathbb{R}^{3}\right),
$$

and we have an explicit formula for the Schwartz kernel of $R_{0}(\lambda)$ :

$$
R_{0}(\lambda, x, y)=\frac{e^{i \lambda|x-y|}}{4 \pi|x-y|} .
$$

(Since $R_{0}(\lambda, x, y)$ depends only on $|x-y|$ due to translation and rotation invariance of $-\Delta$, this can be seen using polar coordinates; see see [80, Theorem 3.3] for a different derivation.)

From this we see that (2.1) makes sense for any $\lambda \in \mathbb{C}$ and any compactly supported $f$ (for instance $f \in L_{\text {comp }}^{2}\left(\mathbb{R}^{3}\right)$ ) and $R_{0}(\lambda) f$ is then a function locally in $L^{2}, R_{0}(\lambda) f \in$ $L_{\text {loc }}^{2}\left(\mathbb{R}^{3}\right)$. In other words, 


$$
R_{0}(\lambda): L^{2}\left(\mathbb{R}^{3}\right) \rightarrow L^{2}\left(\mathbb{R}^{3}\right), \operatorname{Im} \lambda>0
$$

has a holomorphic continuation,

$$
R_{0}(\lambda): L_{\text {comp }}^{2}\left(\mathbb{R}^{3}\right) \rightarrow L_{\text {loc }}^{2}\left(\mathbb{R}^{3}\right), \quad \lambda \in \mathbb{C} .
$$

This, and the connection to the wave equation, explains why we took $\lambda$ as our parameter. The mapping property (2.4) remains valid in all odd dimensions while in even dimensions continuation has to be made to the infinitely sheeted logarithmic plane. In quantum scattering (as opposed to scattering of classical waves) the usual spectral parameter $z=\lambda^{2}$ is more natural in which case the continuation is from $\mathbb{C} \backslash[0, \infty)$ through the cut $[0, \infty)$.

We have the following formula which will be useful later:

$$
\begin{aligned}
R_{0}(\lambda)-R_{0}(-\lambda) & =\frac{i \lambda}{2} \mathbb{E}(\bar{\lambda})^{*} \mathbb{E}(\lambda), \quad \lambda \in \mathbb{C}, \\
\mathbb{E}(\lambda): L^{2}\left(\mathbb{R}^{3}\right) & \rightarrow L_{\operatorname{loc}}^{2}\left(\mathbb{S}^{2}\right), \quad \mathbb{E}(\lambda) g(\omega):=\frac{1}{2 \pi} \int_{\mathbb{R}^{3}} e^{-i \lambda\langle x, \omega\rangle} g(x) d x,
\end{aligned}
$$

where $L^{2}\left(\mathbb{S}^{2}\right)$ is defined using the standard measure on the sphere. This follows from (2.2) and the elementary identity $\int_{\mathbb{S}^{2}} e^{-i\langle y, \omega\rangle} d \omega=-2 i \pi\left(e^{i|y|}-e^{-i|y|}\right) /|y|$, see [80, Lemma 3.2]. In view of the spectral decomposition based on the Fourier transform,

$$
f=\frac{1}{2 \pi} \int_{0}^{\infty} \mathbb{E}(\lambda)^{*} \mathbb{E}(\lambda) f \lambda^{2} d \lambda, \quad-\Delta f=\frac{1}{2 \pi} \int_{0}^{\infty} \lambda^{2} \mathbb{E}(\lambda)^{*} \mathbb{E}(\lambda) f \lambda^{2} d \lambda,
$$

$f \in \mathcal{C}_{\mathrm{c}}^{\infty}\left(\mathbb{R}^{3}\right)$, formula (2.5) is a special case of the Stone formula relating resolvents and spectral measures [80, (B.1.12)].

The spectral decomposition (2.6) and (2.5) is one way to see the relation of $R_{0}(\lambda)$ with the wave equation: for $f \in \mathcal{C}_{\mathrm{c}}^{\infty}\left(\mathbb{R}^{3}\right)$

$$
\begin{aligned}
\frac{\sin t \sqrt{-\Delta}}{\sqrt{-\Delta}} f & =\frac{1}{2 \pi} \int_{0}^{\infty} \sin t \lambda \mathbb{E}(\lambda)^{*} \mathbb{E}(\lambda) f \lambda d \lambda \\
& =\frac{1}{\pi i} \int_{0}^{\infty} \sin t \lambda\left(R_{0}(\lambda)-R_{0}(-\lambda)\right) f d \lambda \\
& =\frac{1}{\pi i} \int_{0}^{\infty} \frac{e^{i t \lambda}-e^{-i t \lambda}}{2 i}\left(R_{0}(\lambda)-R_{0}(-\lambda)\right) f d \lambda \\
& \left.=\frac{1}{2 \pi} \int_{\mathbb{R}} e^{-i t \lambda} R_{0}(\lambda) f d \lambda-\frac{1}{2 \pi} \int_{\mathbb{R}} e^{-i t \lambda} R_{0}(-\lambda)\right) f d \lambda
\end{aligned}
$$

For $t>0$ we can deform $\mathbb{R}$ in the second integral to the contour $\mathbb{R}-i \gamma, \gamma>0$. By letting $\gamma \rightarrow+\infty$ we then see that

$$
\frac{\sin t \sqrt{-\Delta}}{\sqrt{-\Delta}} f=\frac{1}{2 \pi} \int_{\mathbb{R}} e^{-i \lambda t} R_{0}(\lambda) f d \lambda, \quad t>0, \quad f \in \mathcal{C}_{\mathrm{c}}^{\infty}\left(\mathbb{R}^{3}\right) .
$$


(The integrals above converge only in the distributional sense and hence one should first "integrate" both sides against $\varphi(t) \in \mathcal{C}_{\mathrm{c}}^{\infty}((0, \infty))$ and then deform the contour.) When combined with (2.2) we obtain the Schwartz kernel of $\sin (t \sqrt{-\Delta}) / \sqrt{-\Delta}$,

$$
\frac{\sin t \sqrt{-\Delta}}{\sqrt{-\Delta}}(x, y)=\frac{\delta(t-|x-y|)}{4 \pi t}, t>0 .
$$

which gives Kirchhoff's formula

$$
\frac{\sin t \sqrt{-\Delta}}{\sqrt{-\Delta}} f(x)=\frac{1}{4 \pi t} \int_{\partial B(x, t)} f(y) d \sigma(y), \quad t>0 .
$$

The important feature of (2.9) is the support property of the Schwartz kernel: it vanishes outside of the light cone $|x-y|=t$. That is the sharp Huyghens principle valid in all odd dimensions greater than one and violated in even dimensions.

We conclude this section with

Theorem 1 Let $\Delta=\sum_{j=1}^{3} \partial_{x_{j}}^{2}$ be the Laplacian in $\mathbb{R}^{3}$. Then the resolvent

$$
R_{0}(\lambda):=\left(-\Delta-\lambda^{2}\right)^{-1}: L^{2}\left(\mathbb{R}^{3}\right) \rightarrow L^{2}\left(\mathbb{R}^{3}\right), \quad \operatorname{Im} \lambda>0,
$$

extends holomorphically to $\mathbb{C}$ as an operator

$$
R_{0}(\lambda): L_{\text {comp }}^{2}\left(\mathbb{R}^{3}\right) \rightarrow L_{\text {loc }}^{2}\left(\mathbb{R}^{3}\right) .
$$

Moreover, for any $\rho \in \mathcal{C}_{\mathrm{c}}^{\infty}(B(0, R) ;[0,1])$ we have

$$
\left\|\rho R_{0}(\lambda) \rho\right\|_{L^{2} \rightarrow H^{j}} \leq C e^{2 R(\operatorname{Im} \lambda)_{-}}(1+|\lambda|)^{j-1}, \quad j=0,1,
$$

where $C$ depends only on $\rho$.

Proof To see (2.10) we apply the (distributional) Fourier inversion formula to (2.8). That gives

$$
R_{0}(\lambda)=\int_{0}^{\infty} e^{i \lambda t} U(t) d t, \quad U(t):=\frac{\sin t \sqrt{-\Delta}}{\sqrt{-\Delta}} .
$$

Since the Schwartz kernel (2.9) is supported on $|x-y|=t$ and $\rho$ is supported in $|x|<R$ we can also have

$$
\rho R_{0}(\lambda) \rho=\int_{0}^{2 R} e^{i \lambda t} \rho U(t) \rho d t
$$

To use this to prove (2.10), we note that

$$
\begin{aligned}
& \|U(t)\|_{L^{2} \rightarrow H^{1}} \simeq\|U(t)\|_{L^{2} \rightarrow L^{2}}+\|\sqrt{-\Delta} U(t)\|_{L^{2} \rightarrow L^{2}}=\sup _{\lambda \in \mathbb{R}} \frac{|\sin t \lambda|}{|\lambda|}+\sup _{\lambda \in \mathbb{R}}|\sin t \lambda| \\
& \quad=1+|t| .
\end{aligned}
$$


This and (2.11) give the bound (2.10) for $j=1$. For $j=0$ we write (noting that $U(0)=0)$

$$
\lambda \rho R_{0}(\lambda) \rho=\frac{1}{i} \int_{0}^{2 R} \partial_{t}\left(e^{i \lambda t}\right) \rho U(t) \rho d t=-\frac{1}{i} \int_{0}^{2 R} e^{i \lambda t} \rho \partial_{t} U(t) \rho d t .
$$

Since $\partial_{t} U(t)=\cos t \sqrt{-\Delta}$ is uniformly bounded on $L^{2}$, the bound (2.10) for $j=0$ follows.

\subsection{Meromorphic continuation and definition of resonances}

We now consider $P_{V}:=-\Delta+V$ where $V \in L_{\text {comp }}^{\infty}\left(\mathbb{R}^{3}\right)$ is allowed to be complex valued. Since

$$
P_{V}-\lambda^{2}=\left(I+V R_{0}(\lambda)\right)\left(P_{0}-\lambda^{2}\right)
$$

the study of $\left(P_{V}-\lambda^{2}\right)^{-1}$ reduces to the study of $I+V R_{0}(\lambda)$. For $\operatorname{Im} \lambda>0$

$$
\widehat{R_{0}(\lambda) f}(\xi)=\frac{\hat{f}(\xi)}{|\xi|^{2}-\lambda^{2}}
$$

where $f \mapsto \hat{f}$ is the Fourier transform. This implies that

$$
\left\|R_{0}(\lambda)\right\|_{L^{2} \rightarrow L^{2}}=\frac{1}{d\left(\lambda^{2}, \mathbb{R}_{+}\right)} \leq \frac{1}{|\lambda| \operatorname{Im} \lambda}, \quad \operatorname{Im} \lambda>0
$$

It follows that

$$
\operatorname{Im} \lambda \gg 1 \Longrightarrow\left\|V R_{0}(\lambda)\right\|_{L^{2} \rightarrow L^{2}}<1
$$

and hence

$$
\begin{aligned}
R_{V}(\lambda) & :=\left(P_{V}-\lambda^{2}\right)^{-1} \\
& =R_{0}(\lambda)\left(I+V R_{0}(\lambda)\right)^{-1}
\end{aligned}
$$

is a holomorphic family of operators from $L^{2}$ to $L^{2}$ when $\operatorname{Im} \lambda \gg 1$.

We would like to continue $R_{V}(\lambda)$ as a meromorphic family of operators $L_{\text {comp }}^{2}\left(\mathbb{R}^{3}\right) \rightarrow L_{\text {loc }}^{2}\left(\mathbb{R}^{3}\right)$. Because of (2.14) and Theorem 1 this follows from the following statement

$$
\begin{aligned}
& \left(I+V R_{0}(\lambda)\right)^{-1}: L_{\text {comp }}^{2}\left(\mathbb{R}^{3}\right) \rightarrow L_{\text {comp }}^{2}\left(\mathbb{R}^{3}\right) \\
& \quad \text { is a meromorphic family of operators for } \lambda \in \mathbb{C} .
\end{aligned}
$$

Strictly speaking, we should really say that this family is a continuation of the holomorphic family of inverses defined for $\operatorname{Im} \lambda \gg 1$. By a meromorphic family of operators 
$\lambda \mapsto A(\lambda)$ we mean a family which is holomorphic outside a discrete subset of $\mathbb{C}$ and at any point $\lambda_{0}$ in that subset we have

$$
A(\lambda)=\sum_{j=1}^{J} \frac{A_{j}}{\left(\lambda-\lambda_{0}\right)^{j}}+A_{0}(\lambda),
$$

where $A_{j}$ are operators of finite rank and $\lambda \mapsto A_{0}(\lambda)$ is holomorphic near $\lambda_{0}$.

The proof of (2.15) relies on analytic Fredholm theory: suppose that $K(\lambda): L^{2} \rightarrow$ $L^{2}$ is a holomorphic family of compact operators for $\lambda \in \mathbb{C}$ and that $\left(I+K\left(\lambda_{0}\right)\right)^{-1}$ : $L^{2} \rightarrow L^{2}$ exists at some $\lambda_{0} \in \mathbb{C}$. Then

$$
\lambda \mapsto(I+K(\lambda))^{-1} \text { is a meromorphic family operators for } \lambda \in \mathbb{C} .
$$

(See [80, Theorem C.5] for a general statement and a proof.)

It is tempting to apply (2.17) to obtain (2.15) but one immediately notices that $V R_{0}(\lambda)$ is not defined on on $L^{2}$ once $\operatorname{Im} \lambda \leq 0$. To remedy this we introduce $\rho \in$ $\mathcal{C}_{\mathrm{c}}^{\infty}\left(\mathbb{R}^{3}\right)$ equal to 1 on supp $V$ and write

$$
I+V R_{0}(\lambda)=\left(I+V R_{0}(\lambda)(1-\rho)\right)\left(I+V R_{0}(\lambda) \rho\right) .
$$

We consider this first for $\operatorname{Im} \lambda \gg 1$ in which case $R_{0}(\lambda)$ is bounded on $L^{2}$. Then

$$
\left(I+V R_{0}(\lambda)(1-\rho)\right)^{-1}=I-V R_{0}(\lambda)(1-\rho)
$$

is a bounded operator $L^{2} \rightarrow L^{2}$ when $\operatorname{Im} \lambda>0$ and it continues holomorphically to $\mathbb{C}$ as an operator $L_{\text {comp }}^{2} \rightarrow L_{\text {comp }}^{2}$

For $\operatorname{Im} \lambda \gg 1, V R_{0}(\lambda) \rho$ has small norm and we conclude that

$$
\left(I+V R_{0}(\lambda)\right)^{-1}=\left(I+V R_{0}(\lambda) \rho\right)^{-1}\left(I-V R_{0}(\lambda)(1-\rho)\right),
$$

is a bounded operator on $L^{2}$ when $\operatorname{Im} \lambda \gg 1$ and we need to continue it as an operator $L_{\text {comp }}^{2} \rightarrow L_{\text {comp }}^{2}$

Hence to obtain (2.15) we need to show that

$$
\left(I+V R_{0}(\lambda) \rho\right)^{-1}: L_{\text {comp }}^{2}\left(\mathbb{R}^{3}\right) \rightarrow L_{\text {comp }}^{2}\left(\mathbb{R}^{3}\right) \text { is meromorphic in } \lambda \in \mathbb{C} .
$$

But now we can apply (2.17) with $K(\lambda):=V R_{0}(\lambda) \rho$. In fact, (2.10) shows that if supp $\rho \subset B(0, R)$ then $\rho R_{0}(\lambda) \rho: L^{2} \rightarrow H^{1}(B(0, R))$. The Rellich-Kondrachov Theorem [80, Theorem B.3], shows that $\rho R_{0}(\lambda) \rho$ is a compact operator. But then so is $V R_{0}(\lambda) \rho=V\left(\rho R_{0}(\lambda) \rho\right)$. It follows from (2.17) that

$$
\left(I+V R_{0}(\lambda) \rho\right)^{-1}: L^{2}\left(\mathbb{R}^{3}\right) \rightarrow L^{2}\left(\mathbb{R}^{3}\right) \text { is a meromorphic family for } \lambda \in \mathbb{C} \text {. }
$$

To obtain (2.15) we need to show that compactness of the support is preserved by $\left(I+V R_{0}(\lambda) \rho\right)^{-1}$. To see this let $\chi \in \mathcal{C}_{\mathrm{c}}^{\infty}\left(\mathbb{R}^{n}\right)$ be equal to 1 on supp $\rho$. We claim that 


$$
\left(I+V R_{0}(\lambda) \rho\right)^{-1} \chi=\chi\left(I+V R_{0}(\lambda) \rho\right)^{-1} .
$$

In fact, this is valid for $\operatorname{Im} \lambda \gg 1$ by expanding the inverse in Neumann series and then it follows by analytic continuation. From (2.20) and (2.21) we obtain (2.19) which then gives (2.15).

Combining (2.14) and (2.18) with (2.15) we proved

Theorem 2 The operator $R_{V}(\lambda)$ defined in (2.14) continues to a meromorphic family

$$
R_{V}(\lambda): L_{\text {comp }}^{2}\left(\mathbb{R}^{3}\right) \longrightarrow L_{\text {loc }}^{2}\left(\mathbb{R}^{3}\right), \quad \lambda \in \mathbb{C} .
$$

This gives a mathematical definition of scattering resonances:

Definition 1 Suppose that $V \in L_{\text {comp }}^{\infty}\left(\mathbb{R}^{3}\right)$ and that $R_{V}(\lambda)$ is the scattering resolvent of Theorem 2. The poles of $\lambda \mapsto R_{V}(\lambda)$ are called scattering resonances of $V$. If $\lambda_{0}$ is a scattering resonance then, in the notation of (2.16) with $A(\lambda)=R_{0}(\lambda)$, the multiplicity of $\lambda_{0}$ is defined as

$$
m\left(\lambda_{0}\right)=\operatorname{dim} \operatorname{span}\left\{A_{1}\left(L_{\text {comp }}^{2}\right), \ldots, A_{J}\left(L_{\text {comp }}^{2}\right)\right\} .
$$

There are other equivalent definitions of multiplicity which use the special structure of $A_{j}$ 's in the case of a resolvent. For instance for $\lambda_{0} \neq 0$,

$$
\begin{aligned}
m\left(\lambda_{0}\right) & =\operatorname{rank} A_{1} \\
& =\operatorname{rank} \oint_{\lambda_{0}} R_{V}(\lambda) 2 \lambda d \lambda,
\end{aligned}
$$

where the integral is over a small circle containing $\lambda_{0}$ but no other pole of $R_{V}$. The situation at 0 is more complicated — see [80, 33.3$]$ which can serve as an introduction to general theory of Jensen-Kato [149] and Jensen-Nenciu [150].

To see the validity of (2.22) we use the equation $\left(P-\lambda^{2}\right) R_{V}(\lambda) f=f, f \in L_{\text {comp }}^{2}$, to see that near a pole $\lambda_{0} \neq 0$,

$$
\begin{aligned}
R_{V}(\lambda) & =\sum_{j=1}^{J} \frac{\left(P_{V}-\lambda_{0}^{2}\right)^{j-1} \Pi_{\lambda_{0}}}{\left(\lambda^{2}-\lambda_{0}^{2}\right)^{j}}+A\left(\lambda, \lambda_{0}\right), \\
\Pi_{\lambda_{0}} & =\frac{1}{2 \pi i} \oint_{\lambda_{0}} R_{V}(\lambda) 2 \lambda d \lambda,
\end{aligned}
$$

where $\lambda \mapsto A\left(\lambda, \lambda_{0}\right)$ is holomorphic near $\lambda_{0},\left(P_{V}-\lambda_{0}^{2}\right)^{J} \Pi_{\lambda_{0}}=0$ and $P_{V}-\lambda_{0}^{2}$ : $\Pi_{\lambda_{0}}\left(L_{\text {comp }}^{2}\right) \rightarrow \Pi_{\lambda_{0}}\left(L_{\text {comp }}^{2}\right)$ - see [80, $\left.33.2, \S 4.2\right]$. This leads to

Definition 2 A function $u \in \Pi_{\lambda_{0}}\left(L_{\text {comp }}^{2}\left(\mathbb{R}^{3}\right)\right) \subset L_{\text {loc }}^{2}\left(\mathbb{R}^{3}\right)$ is called a generalized resonant state. If $\left(P_{V}-\lambda_{0}^{2}\right) u=0$ then $u$ is called a resonant state. This is equivalent to $u \in\left(P_{V}-\lambda_{0}^{2}\right)^{J-1} \Pi_{\lambda_{0}}\left(L_{\text {comp }}^{2}\left(\mathbb{R}^{3}\right)\right)$. 
Formula (2.14) and the expansion (2.23) give the following characterization (see [80, Theorem 3.7, Theorem 4.9])

$\mathrm{u}$ is a resonant state for a resonance $\lambda_{0}$

$$
\exists f \in L_{\text {comp }}^{2}\left(\mathbb{R}^{3}\right) u=R_{0}\left(\lambda_{0}\right) f,\left(P_{V}-\lambda_{0}^{2}\right) u=0 .
$$

The condition $u=R_{0}(\lambda) f$ is called the outgoing condition. In Sect. 2.6 we will see a more complicated but much more natural characterization of outgoing states.

When $V$ is real valued then $P_{V}$ is a self-adjoint operator (with the domain given by $H^{2}\left(\mathbb{R}^{3}\right)$ ). The poles of $R_{V}(\lambda)$ in $\operatorname{Im} \lambda>0$ correspond to negative eigenvalues of $P_{V}$ and in (2.23) we have $J=1$. This of course is consistent with (2.24) since for $\operatorname{Im} \lambda_{0}>0, R_{0}\left(\lambda_{0}\right) f \in L^{2}\left(\mathbb{R}^{3}\right)$.

\subsection{Upper bound on the number of resonances}

Once we have defined resonances it is natural to estimate the number of resonances. Since they are now in the complex plane the basic counting function is given by

$$
N_{V}(r):=\sum\{m(\lambda):|\lambda| \leq r\}
$$

where $m(\lambda)$ is defined in (2.22).

We will prove the following optimal bound

Theorem 3 Suppose that $V \in L_{\text {comp }}^{\infty}\left(\mathbb{R}^{3}\right)$. Then

$$
N_{V}(r) \leq C r^{3}
$$

The bound, $N_{V}(r) \leq C r^{n}$, valid in all odd dimensions $n$, was proved in [278] using methods developed by Melrose [181,182] who proved $N_{V}(r) \leq C r^{n+1}$. The proof presented here uses a substantial simplification of the argument due to Vodev [265] who proved corresponding upper bounds in even dimension [266,267], following an earlier contribution by Intissar [142]. When $n=1$ we have an asymptotic formula,

$$
N_{V}(r)=\frac{2}{\pi} \operatorname{diam}(\operatorname{supp} V) r+o(r),
$$

stated by Regge [221] and proved in [276].

The exponent 3 in (2.26) is optimal as shown by the case of radial potentials. When $V(x)=v(|x|)(R-|x|)_{+}^{0}$, where $v$ is a $C^{2}$ even function, and $v(R)>0$, then, see [277],

$$
N_{V}(r)=C_{R} r^{3}+o\left(r^{3}\right)
$$

The constant $C_{R}$ and its appearance in a refinement of (2.26) is explained by Stefanov [255]. 
Interpretation. In the case of $-\Delta+V$ on a bounded domain or a closed manifold, for instance on $M=\mathbb{T}^{n}:=\mathbb{R}^{n} / \mathbb{Z}^{n}$ or $M=\mathbb{S}^{n}$ (the $n$-sphere), the spectrum is discrete and for $V \in L^{\infty}\left(\mathbb{T}^{n} ; \mathbb{R}\right)$ we have the asymptotic Weyl law for the number of eigenvalues:

$$
\begin{aligned}
& \left|\left\{\lambda: \lambda^{2} \in \operatorname{Spec}\left(-\Delta_{M}+V\right),|\lambda| \leq r\right\}\right|=c_{n} \operatorname{vol}(M) r^{n}+o\left(r^{n}\right), \\
& \quad c_{n}=2 \operatorname{vol}\left(B_{\mathbb{R}^{n}}(0,1)\right) /(2 \pi)^{n},
\end{aligned}
$$

where the eigenvalues are included according to their multiplicities.

In the case of $-\Delta+V$ on $\mathbb{R}^{n}$, the discrete spectrum is replaced by the discrete set of resonances. Hence the bound (2.26) is an analogue of the Weyl law. Except in dimension one-see (2.27) — the issue of asymptotics or even optimal lower bounds remains unclear at the time of writing of this survey. We will discuss lower bounds and existence of resonances in Sect. 2.7.

The strategy for the proof of the upper bound (2.26) is to include poles of $R_{V}(\lambda)$ among zeros of an entire function. The number of poles is then estimated by estimating the growth of that function and using Jensen's inequality — see the proof of Theorem 3 below. In particular, if $h(\lambda)$ is our entire function then (2.26) follows from a bound

$$
|h(\lambda)| \leq A e^{A|\lambda|^{3}}
$$

for some constant $A$.

Hence our goal is to find a suitable $h$ and to prove (2.30). Before doing it we recall some basic facts about trace class operators and Fredholm determinants. We refer to [80, Appendix B] for proofs and pointers to the literature.

Suppose that $A: H_{1} \rightarrow H_{2}$ is a bounded operator between two Hilbert spaces. Then $\left(A^{*} A\right)^{\frac{1}{2}}: H_{1} \rightarrow H_{1}$ is a bounded non-negative self-adjoint operator and we denote its spectrum by $\left\{s_{j}(A)\right\}_{j=0}^{\infty}$. The numbers $s_{j}(A)$ are called singular values of $A$. We will need the following well known inequalities [80, Proposition B.15]

$$
s_{j+k}(A+B) \leq s_{j}(A)+s_{k}(B), \quad s_{j+k}(A B) \leq s_{j}(A) s_{k}(B) .
$$

If $H_{1}=H_{2}=L^{2}\left(\mathbb{R}^{3}\right)$ and in addition $A: L^{2}\left(\mathbb{R}^{3}\right) \rightarrow H^{s}\left(\mathbb{R}^{3}\right), s \geq 0$, and the support of the Schwartz kernel of $A$ is contained in $B(0, R) \times B(0, R)$, then, with a constant depending on $R$,

$$
s_{j}(A) \leq C_{R}\|A\|_{L^{2}\left(\mathbb{R}^{3}\right) \rightarrow H^{s}\left(\mathbb{R}^{3}\right)}(1+j)^{-s / 3} .
$$

(See [80, Example after Proposition B.16] for a proof valid in all dimensions $n$, that is with 3 replaced by $n$ in (2.32).)

The operator $A$ is said to be of trace class, $A \in \mathcal{L}_{1}\left(H_{1}, H_{2}\right)$, if

$$
\|A\|_{\mathcal{L}_{1}}:=\sum_{j=0}^{\infty} s_{j}(A)<\infty .
$$


For $A \in \mathcal{L}_{1}\left(H_{1}, H_{1}\right)$ can we define Fredholm determinant, $\operatorname{det}(I+A)$,

$$
|\operatorname{det}(I+A)| \leq e^{\|A\|_{\mathcal{L}_{1}}}
$$

see [80, §B.5]. A more precise statement is provided by a Weyl inequality (see [80, Proposition B.24])

$$
|\operatorname{det}(I+A)| \leq \prod_{j=0}^{\infty}\left(1+s_{j}(A)\right) .
$$

Lidskii's theorem [80, Proposition B.27] states that

$$
\operatorname{det}(I+A)=\prod_{j=0}^{\infty}\left(1+\lambda_{j}(A)\right),
$$

where $\left\{\lambda_{j}(A)\right\}_{j=0}^{\infty}$ are the eigenvalues of $A$. In particular, $\operatorname{det}(I+A)=0$ if and only if $I+A$ is not invertible. When $\lambda \mapsto A(\lambda)$ is a holomorphic family of operators a more precise statement can be made by taking account multiplicities-see [80, §C.4].

We will now take $A=-\left(V R_{0}(\lambda) \rho\right)^{4}, H_{1}=L^{2}\left(\mathbb{R}^{3}\right)$. From (2.10), (2.31) and (2.32) we see that

$$
s_{j}\left(V R_{0}(\lambda) \rho\right) \leq\|V\| s_{j}\left(\rho R_{0}(\lambda) \rho\right) \leq C e^{C(\operatorname{Im} \lambda)_{-}}(1+j)^{-1 / 3} .
$$

Another application of (2.31) shows that

$$
s_{j}\left(\left(V R_{0}(\lambda) \rho\right)^{4}\right) \leq C e^{C(\operatorname{Im} \lambda)_{-}}(1+j)^{-4 / 3},
$$

and hence $\left(V R_{0}(\lambda) \rho\right)^{4} \in \mathcal{L}_{1}$. Thus we can define

$$
h(\lambda):=\operatorname{det}\left(I-\left(V R_{0}(\lambda) \rho\right)^{4}\right) .
$$

We have

$$
I-\left(V R_{0}(\lambda) \rho\right)^{4}=\left(I-V R_{0}(\lambda) \rho+\left(V R_{0}(\lambda) \rho\right)^{2}-\left(V R_{0}(\lambda) \rho\right)^{3}\right)\left(I+V R_{0}(\lambda) \rho\right) .
$$

Hence in view of (2.14) and (2.18) it is easy to believe that the poles of $R_{V}(\lambda)$ are included, with multiplicities, among the zeros of $h(\lambda)$ —see [80, Theorem 3.23]:

$$
m_{V}\left(\lambda_{0}\right) \leq \frac{1}{2 \pi i} \oint_{\lambda_{0}} \frac{h^{\prime}(\lambda)}{h(\lambda)} d \lambda
$$

Remark It is not difficult to see that we could, just as in [278], take $\operatorname{det}(I-$ $\left.\left(V R_{0}(\lambda) \rho\right)^{2}\right)$. But the power 4 makes some estimates more straightforward. We could also have taken a modified determinant of $I+V R_{0}(\lambda) \rho[80$, Theorem 3.23, §B.7] and that would give an entire function whose zeros are exactly the resonances of $V$. That 
works in dimension three but in higher dimensions the modified determinants grow faster than (2.30)—see [278].

Proof of Theorem 3 Jensen's formula relates the number of zeros of an entire function $h$ to its growth: if $n(r)$ is the number of zeros of $h$ in $|z| \leq r$

$$
\int_{0}^{r} \frac{n(t)}{t} d t=\frac{1}{2 \pi} \int_{0}^{2 \pi} \log \left|h\left(r e^{i \theta}\right)\right| d \theta-\log |h(0)|,
$$

where we assumed that $h(0) \neq 0$ (an easy modification takes care of the general case). By changing $r$ to $2 r$, it follows that

$$
n(r) \leq \frac{1}{\log 2} \sup _{|\lambda| \leq 2 r} \log |h(\lambda)|-\log |h(0)| .
$$

It follows from this and (2.38) that

$$
|h(\lambda)| \leq A e^{A|\lambda|^{3}} \Longrightarrow n(r) \leq C r^{3} \Longrightarrow N_{V}(r) \leq C r^{3}
$$

To prove the bound on $h$ we first note that (2.36) and (2.33) show that $|h(\lambda)| \leq C$ for $\operatorname{Im} \lambda \geq 0$. To get a good estimate for $\operatorname{Im} \lambda \leq 0$ we use (2.31) and (2.34):

$$
|h(\lambda)| \leq \prod_{k=0}^{\infty}\left(1+s_{k}\left(\left(V R_{0}(\lambda) \rho\right)^{4}\right)\right) \leq \prod_{k=0}^{\infty}\left(1+\|V\|_{L^{\infty} s_{[k / 4]}}^{4}\left(\rho R_{0}(\lambda) \rho\right)^{4}\right)
$$

Hence we need to estimate $s_{j}\left(\rho R_{0}(\lambda) \rho\right)$ for $\rho \in \mathcal{C}_{\mathrm{c}}^{\infty}\left(\mathbb{R}^{n}\right)$.

For $\operatorname{Im} \lambda \geq 0$ we already have the estimate (2.35). To obtain estimates for $\operatorname{Im} \lambda \leq 0$ we use (2.5) to write

$$
\rho\left(R_{0}(\lambda)-R_{0}(-\lambda)\right) \rho=\frac{i \lambda}{2} \rho \mathbb{E}(\bar{\lambda})^{*} \mathbb{E}(\lambda) \rho
$$

Hence, using (2.31) and (2.35) again,

$$
\begin{aligned}
s_{j}\left(\rho R_{0}(\lambda) \rho\right) & \leq C|\lambda|\|E(\lambda) \rho\| s_{[j / 2]}(\mathbb{E}(\lambda) \rho)+s_{[j / 2]}\left(\rho R_{0}(-\lambda) \rho\right) \\
& \leq C \exp (C|\lambda|) s_{[j / 2]}(\mathbb{E}(\lambda) \rho)+C(1+j)^{-1 / 3} .
\end{aligned}
$$

To estimate $s_{j}(\mathbb{E}(\lambda) \rho)$ we use the Laplacian on the sphere, $-\Delta_{\mathbb{S}^{2}}$ and the estimate:

$$
\begin{aligned}
\left\|\left(-\Delta_{\mathbb{S}^{2}}+1\right)^{\ell} \mathbb{E}(\lambda) \rho\right\| & \leq C \sup _{\omega \in \mathbb{S}^{n-1},|x| \leq R}\left|\left(-\Delta_{\omega}+1\right)^{\ell} e^{i \lambda\langle x, \omega\rangle}\right| \\
& \leq C^{\ell} \exp (C|\lambda|)(2 \ell) !
\end{aligned}
$$

where we estimated the supremum using the Cauchy estimates and assumed that supp $\rho \subset B(0, R)$. (We follow the usual practice of changing the value of $C$ from line to line.) 
From the explicit formula for the eigenvalues of $-\Delta_{\mathbb{S}^{2}}$ (given by $k(k+1)$ with multiplicity $k+1$ ), or from the general counting law (2.29), we see that

$$
s_{j}\left(\left(-\Delta_{\mathbb{S}^{2}}+1\right)^{-\ell}\right) \leq C^{\ell}(1+j)^{-\ell} .
$$

The combination of the last two estimates gives

$$
\begin{aligned}
s_{j}(\mathbb{E}(\lambda) \rho) & \leq s_{j}\left(\left(-\Delta_{\mathbb{S}^{2}}+1\right)^{-\ell}\right)\left\|\left(-\Delta_{\mathbb{S}^{2}}+1\right)^{\ell} E_{\rho}(\lambda)\right\| \\
& \leq C^{\ell}(1+j)^{-\ell} \exp (C|\lambda|)(2 \ell) !
\end{aligned}
$$

We now optimize the estimate (2.41) in $\ell$ which gives

$$
s_{j}(\mathbb{E}(\lambda) \rho) \leq C \exp \left(C|\lambda|-j^{\frac{1}{2}} / C\right) .
$$

Going back to (2.40) we obtain

$$
\begin{aligned}
s_{j}\left(V R_{0}(\lambda) \rho\right) & \leq C \exp \left(C|\lambda|-j^{\frac{1}{2}} / C\right)+C(1+j)^{-\frac{1}{3}} \\
& \leq \begin{cases}e^{C|\lambda|+C}, & j \leq C|\lambda|^{2} \\
C(1+j)^{-\frac{1}{3}}, & j \geq C|\lambda|^{2}\end{cases}
\end{aligned}
$$

Returning to (2.39) we use (2.43) as follows

$$
|h(\lambda)| \leq \prod_{k \leq C|\lambda|^{2}} e^{C|\lambda|+C}\left(\exp \sum_{k \geq C|\lambda|^{2}} k^{-4 / 3}\right) \leq C e^{C|\lambda|^{3}},
$$

which completes the proof.

The key to fighting exponential growth when $\operatorname{Im} \lambda<0$ is analyticity near infinity which is used implicitely in (2.41)-(2.44). That is a recurrent theme in many approaches to the study of resonances-see 2.6.

Resonance counting has moved on significantly since these early results. Some of the recent advances will be reviewed in Sect. 3.4, see also [279] for an account of other early works. The most significant breakthrough was Sjöstrand's discovery [240] of geometric upper bounds on the number of resonances in which the exponent is no longer the dimension as in (2.26) and (2.29) but depends on the dynamical properties of the classical system.

\subsection{Resonance free regions}

The imaginary parts of resonances are interpreted as decay rates of the corresponding resonant states-see Sect. 2.5 for a justification of that in the context of the wave equation. If there exists a resonance closest to the real axis then (assuming we can 
justify expansions like (1.2)—see Theorems 5,12) its imaginary part determines the principal rate of decay of waves - see the end of this section for some comments on that. If waves are localized in frequency then imaginary parts of resonances with real parts near that frequency should determine the decay of those waves.

But to assure the possibility of having a principal resonance, that is a resonance closest to the real axis, we need to know that there exists a strip without any resonances. Hence it is of interest to study high frequency resonance free regions which are typically of the form

$$
\operatorname{Im} \lambda>-F(\operatorname{Re} \lambda), \operatorname{Re} \lambda>C, \quad F(x)=\left\{\begin{array}{l}
(a) e^{-\alpha x}, \alpha>0 \\
(b) M \\
(c) M \log x \\
(d) \gamma x^{\beta}, \beta \in \mathbb{R}, \gamma>0
\end{array}\right.
$$

where $M>0$ may be fixed or arbitrarily large. In the setting of compactly supported potentials we see cases of $(c)$ and $(d)$ : fixed $M$ for bounded potentials [162], arbitrary $M$ for smooth potentials [262] and $\beta=1 / a$ for potentials in the $a$-Gevrey class [110]. For applications it is important that the statement about resonance free region is quantitative which means that it comes with some resolvent bounds-see (2.46) below and for applications Sect. 2.5.

Here we present the simplest case:

Theorem 4 Suppose that $V \in L^{\infty}\left(\mathbb{R}^{3}\right)$, supp $V \subset B\left(0, R_{0}\right)$, and that $R_{V}(\lambda)$ is the scattering resolvent of Theorem 2 .

Then we can find $A>0$ such that for any $\chi \in \mathcal{C}_{\mathrm{c}}^{\infty}\left(B\left(0, R_{1}\right)\right), R_{1} \geq R_{0}$, there exists $C$ for which

$$
\left\|\chi R_{V}(\lambda) \chi\right\|_{L^{2} \rightarrow H^{j}} \leq C(1+|\lambda|)^{j-1} e^{2 R_{1}(\operatorname{Im} \lambda)_{-}}, \quad j=0,1,
$$

when $\operatorname{Im} \lambda>-\frac{1}{2 R_{0}} \log |\operatorname{Re} \lambda|+A$.

Proof Without loss of generality we can take $\chi$ which is equal to 1 on the support of $\rho$ used in (2.19). Then using Theorem 1, (2.14), (2.18) and (2.21) we see that

$$
\begin{aligned}
\left\|\chi R_{V}(\lambda) \chi\right\|_{L^{2} \rightarrow H^{j}} & =\left\|\chi R_{0}(\lambda) \chi\left(I+V R_{0}(\lambda) \rho\right)^{-1}\left(I-V \chi R_{0}(\lambda) \chi(1-\rho)\right)\right\|_{L^{2} \rightarrow H^{j}} \\
& \leq\left\|\chi R_{0}(\lambda) \chi\right\|_{L^{2} \rightarrow H^{j}}\left(1+\|V\|_{\infty}\left\|\chi R_{0}(\lambda) \chi\right\|\right)\left\|\left(I+V R_{0}(\lambda) \rho\right)^{-1}\right\| \\
& \leq C_{1}(1+|\lambda|)^{j-1} e^{4 R_{1}(\operatorname{Im} \lambda)_{-}}\left\|\left(I+V R_{0}(\lambda) \rho\right)^{-1}\right\|,
\end{aligned}
$$

where the norms are operator norms $L^{2} \rightarrow L^{2}$ unless indicated otherwise. Assuming, as we may that, supp $\rho \subset B\left(0, R_{0}\right)$ (the only requirement on $\rho \in \mathcal{C}_{\mathrm{c}}^{\infty}\left(\mathbb{R}^{3}\right)$ is that it is equal to 1 on the support of $V$ ), it is sufficient to prove that there exist $A$ such that

$$
\left\|\left(I+V R_{0}(\lambda) \rho\right)^{-1}\right\|_{L^{2} \rightarrow L^{2}}<2 \text { when } \operatorname{Im} \lambda>-\frac{1}{2 R_{0}} \log |\operatorname{Re} \lambda|+A .
$$


However, (2.10) applied with $j=0$ gives

$$
\left\|V R_{0}(\lambda) \rho\right\| \leq C_{2}(1+|\lambda|)^{-1} e^{2 R_{0}(\operatorname{Im} \lambda)_{-}}<\frac{1}{2},
$$

once $A$ is large enough (depending on $C_{2}$ which depends on $\rho$ and $V$ ). This completes the proof as we can then invert $I+V R_{0}(\lambda) \rho$ as a Neumann series.

In the setting of obstacle scattering, (see Fig. 15 for an example and [80, §§4.1,4.2] for a general framework which includes this case) the study of resonance free regions and of the closely related decay of waves was initiated by Lax-Phillips [162], Morawetz [187] and Ralston [218], see also Morawetz-Ralston-Strauss [189]. These early results stressed the importance of the billiard flow ${ }^{1}$ and provided an impetus for the study of propagation of singularities for boundary value problems by Andersson, Ivrii, Melrose, Lebeau, Sjöstrand and Taylor—see Hörmander [137, Chapter 24] and references given there. A direct method, alternative to Lax-Phillips theory, for relating propagation of singularities to resonance free strips was developed by Vainberg [262], $[80, \S 4.6]$. All the possibilities in (2.45) can occur in the case of scattering by obstacles with smooth boundaries:

\begin{tabular}{ll}
\hline Geometry & Resonance free region \\
\hline Arbitrary obstacle & (a) [37]; optimal for obstacles with elliptic closed orbits [254,256,260] \\
Two convex obstacles & (b) with a pseudo-lattice of resonances below the strip [104,139], \\
hence optimal & (b) with $M$ determined by a topological pressure of the chaotic system \\
Several convex obstacles & [103,140]; an improved gap [210] \\
& (c) with arbitrarily large $M$ [179,262], using propagation of \\
Smooth non-trapping & singularities [137, Chapter 24]; \\
obstacles & (d) with $\beta=\frac{1}{3}[12,215]$ based on propagation of Gevrey-3 \\
Analytic non-trapping & singularities [164]; optimal for convex obstacles \\
obstacles & (d) with $\beta=\frac{1}{3}$ and $\gamma$ determined by the curvature $[124,151,246] ;$ \\
Smooth strictly convex & Weyl asymptotics for resonances in cubic bands $\sim r^{n-1}[247]$ \\
\hline obstacles &
\end{tabular}

There has been recent progress in the study of non-smooth obstacles, and in particular in taking account of the effect of diffraction at conic points on the distribution of resonances-see the lecture by Wunsch [272] for a survey and references.

Another rich set of recent results concerns scattering by "thin" barriers modeled by delta function potentials (possibly energy dependent) supported on hypersurfaces in $\mathbb{R}^{n}$. For example, refinements of the Melrose-Taylor parametrix techniques give, in some cases, the optimal resonance free region defined by $F(x)=x^{-\beta}, \beta>0$ and a mathematical explanation of a Sabine law for quantum corrals [17]. See the works of Galkowski [96-98] and Smith-Galkowski [100] where references to earlier literature on resonances for transmission problems can also be found, and Fig. 14 for an illustration.

\footnotetext{
1 That is the flow defined by propagation along straight lines with reflection at the boundary; trapping refers to existence of trajectories which never escape to infinity.
} 
Fig. 14 An illustration of the results of $[96,98]$. At the top an image of a quantum corral formed from individual iron atoms taken using a scanning tunneling microscope; this motivated the quantum Sabine law of [17] where other numerical experiments can be found. In the middle resonances computed for a simple model of a circular quantum corral: scattering by a delta function of the form $\delta_{\mathbb{S} 1}$ where $\mathbb{S}^{1} \subset \mathbb{R}^{2}$ is the unit circle. It shows resonances and the bound on the resonance free region predicted by the Sabine law (the dashed black line). To understand the Sabine law, consider, $u_{0}$, a function localized in space and momentum to $\left(x_{0}, \xi_{0}\right) \in \Omega \times \mathbb{S}^{1}$ up to the scale allowed by the uncertainty principle (a "wave packet"). A wave started with initial data $u_{0}$ propagates along the billiard flow starting from $\left(x_{0}, \xi_{0}\right)$. At each intersection of the billiard flow with the boundary, the amplitude inside of $\Omega$ will decay by a factor, $R$, depending on the point and direction of intersection. Suppose that the billiard flow from $\left(x_{0}, \xi_{0}\right)$ intersects the boundary at $\left(x_{n}, \xi_{n}\right) \in \partial \Omega \times \mathbb{S}^{1}$, $n>0$. Let $l_{n}=\left|x_{n+1}-x_{n}\right|$ be the distance between two consecutive intersections with the boundary, as shown at the bottom figure. Then the amplitude of the wave decays by a factor $\prod_{i=1}^{n} R_{i}$ in time $\sum_{i=1}^{n} l_{i}$ where $R_{i}=R\left(x_{i}, \xi_{i}\right)$. The energy scales as amplitude squared and since the imaginary part of a resonance gives the exponential decay rate of $L^{2}$ norm, this leads us to the heuristic that resonances should occur at $\operatorname{Im} \lambda=\overline{\log |R|^{2}} /(2 \bar{l})$ where the mean is defined by $\bar{f}=\frac{1}{N} \sum_{i=1}^{N} f_{i}$. We call this heuristic rule the quantum Sabine law
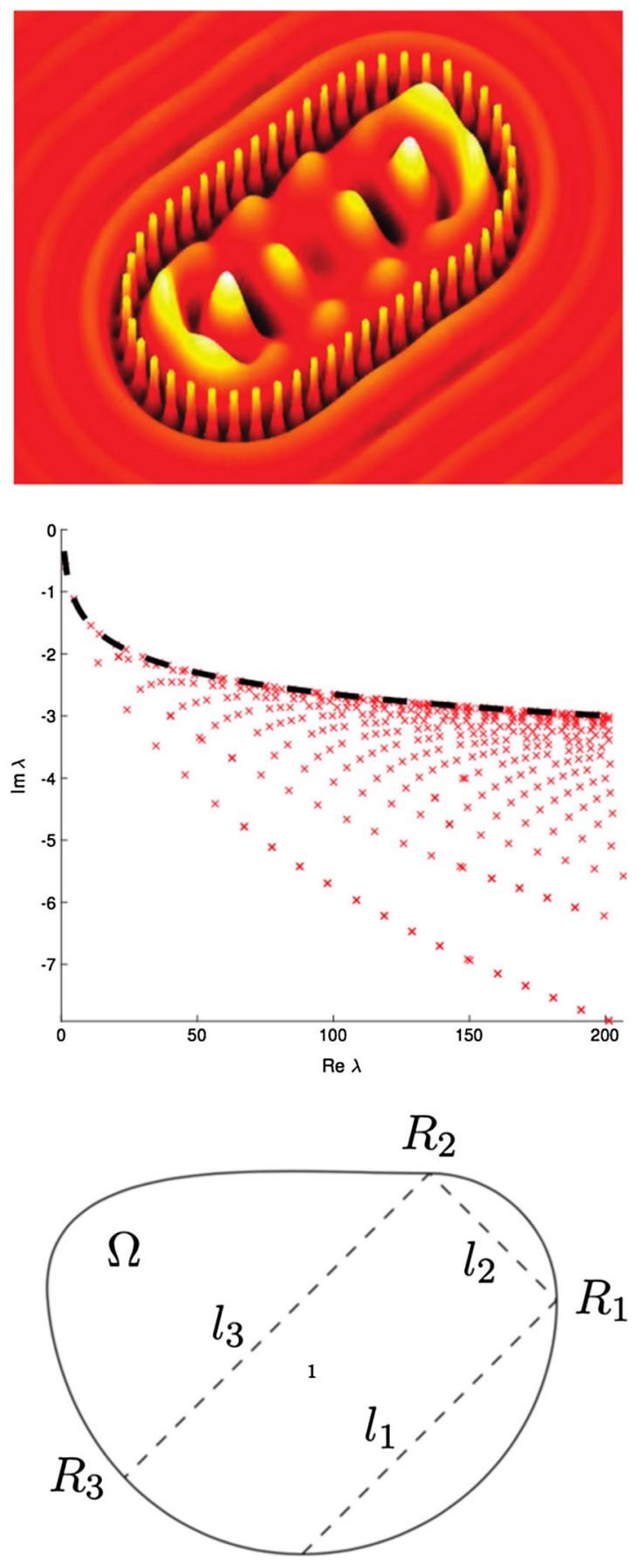

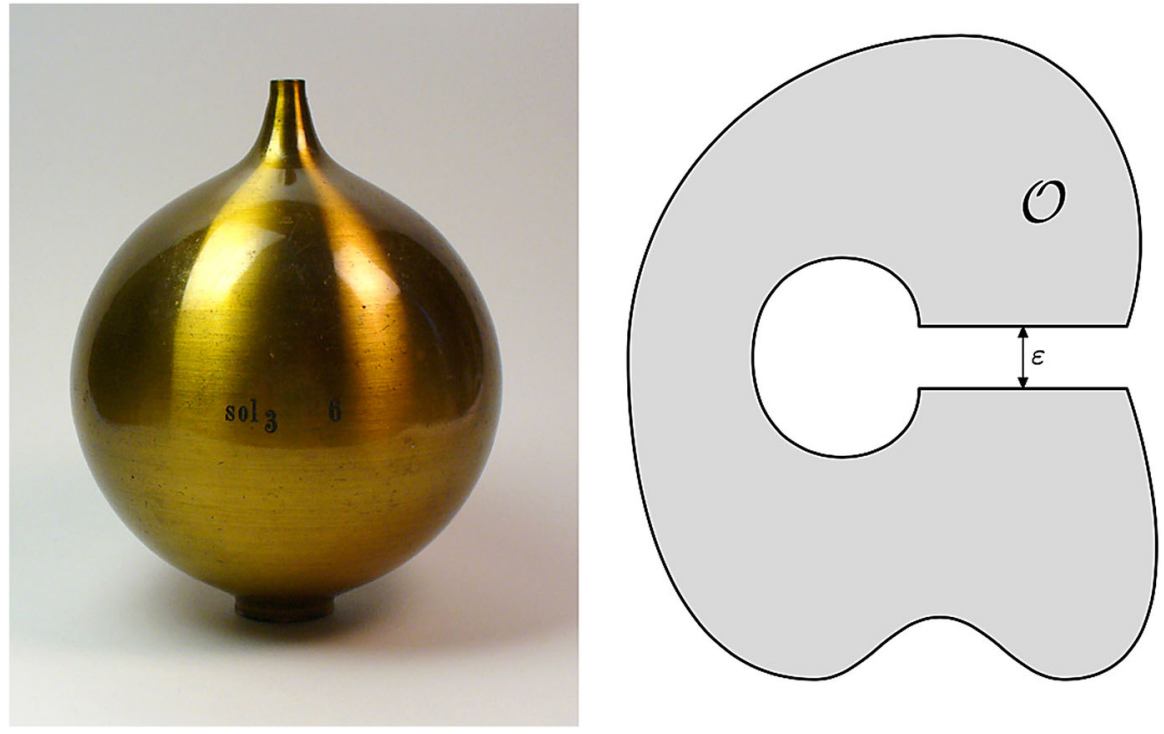

Fig. 15 Left an actual Helmholtz resonator (reproduced from https://en.wikipedia.org/wiki/ Helmholtz_resonance under the Creative Commons licence). Right a general mathematical model allowing for arbitrary cavities and exteriors [64]. The shaded part is the obstacle $\mathcal{O}$ and resonances are the values of $\lambda$ for which there exists a solution to $\left(-\Delta-\lambda^{2}\right) u=0$ in $\mathbb{R}^{n} \backslash \mathcal{O},\left.u\right|_{\partial \mathcal{O}}=0$ which is outgoing, that is, $\left.u\right|_{\mathbb{R}^{n} \backslash B(0, R)}=\left.R_{0}(\lambda) f\right|_{\mathbb{R}^{n} \backslash B(0, R)}$ for some $f \in L_{\text {comp }}^{2}\left(\mathbb{R}^{n}\right)$ and $R \gg 1$. This is also an example in which high energy quasimodes supported in the cavity provide an approximation to resonances $[254,260]$ with imaginary parts $\mathcal{O}\left(e^{-c|\operatorname{Re} \lambda|}\right)$

Finally we make some comments on low energy bounds on resonance widths. Universal lower bounds bounds on $|\operatorname{Im} \lambda|$ for star shaped obstacles were obtained by Morawetz [187,188] for obstacle problems and by Fernández-Lavine [91] for more general operators. In the case of star shaped obstacles the best bound is due to Ralston [219] who using Lax-Phillips theory [162] showed that in all odd dimensions

$$
\operatorname{Im} \lambda<-2 \operatorname{diam}(\mathcal{O})^{-1} .
$$

Remarkably this bound is optimal for the sphere in dimensions three and five. The obvious difficulty in obtaining such bounds is the lack of a variational principle due to the non-self-adjoint nature of the problem (see Sect. 2.6).

The opposite extreme of star shaped obstacles are Helmholtz resonators shown in Fig. 15. Here a cavity is connected to the exterior through a neck of width $\varepsilon$. A recent breakthrough by Duyckaerts-Grigis-Martinez [64] provided precise asymptotics (as $\varepsilon \rightarrow 0$ ) of the resonance width associated to the lowest mode of the cavity.

\subsection{Resonance expansions of scattered waves}

We will use Theorem 4 and prove an expansion of solutions to the wave equation in terms of resonances-see (1.2) and Figs. 6 and 7. To simplify the presentation we make the following assumption: 
$P_{V}$ has no eigenvalues and 0 is not a resonance;

all resonances of $P_{V}$ are simple ${ }^{2}$,

see $[80, \S 2.2,3.2 .2]$ for the general case and $[80, \S 7.5]$ for resonance expansions in the case where trapping is allowed.

Theorem 5 Suppose that $V \in L^{\infty}\left(\mathbb{R}^{3}\right)$ is real valued, supp $V \subset B\left(0, R_{0}\right)$ and that the assumptions above holds. Let $w(t, x)$ be the solution of

$$
\left\{\begin{array}{l}
\left(\partial_{t}^{2}+P_{V}\right) w(t, x)=0 \\
w(0, x)=f(x) \in H^{1}\left(B\left(0, R_{1}\right)\right) \\
\partial_{t} w(0, x)=g(x) \in L^{2}\left(B\left(0, R_{1}\right)\right)
\end{array}\right.
$$

where $R_{1} \geq R_{0}$. Then for any $a>0$,

$$
w(t, x)=\sum_{\operatorname{Im} \lambda_{j}>-a} e^{-i \lambda_{j} t} w_{j}(x)+E_{a}(t),
$$

where $\left\{\lambda_{j}\right\}_{j=1}^{\infty}$ are the resonances of $P_{V}$ and $w_{j}$ are the corresponding resonant states,

$$
w_{j}=\operatorname{Res}_{\lambda=\lambda_{j}}\left(i R_{V}(\lambda) g+\lambda R_{V}(\lambda) f\right) .
$$

exists a constants $C$ depending on $V, R_{1}$ and a such that

$$
\left\|E_{a}(t)\right\|_{H^{1}\left(B\left(0, R_{1}\right)\right)} \leq C e^{-t a}\left(\|f\|_{H^{1}}+\|g\|_{L^{2}}\right), \quad t \geq 0 .
$$

Interpretation. Suppose that instead of solving the wave equation (2.48) with $x \in \mathbb{R}^{3}$ we consider it for $x \in \Omega \Subset \mathbb{R}^{3}$, with, say, Dirichlet boundary conditions, $u(x, t)=0$, $x \in \partial \Omega$ (for $\partial \Omega$ smooth). Then the solution can be expanded in a series of eigenfunctions of the Dirichlet realization of $P_{V}$. Let us assume for simplicity that all eigenvalues are positive, $0<\lambda_{1}^{2} \leq \lambda_{2}^{2} \cdots \leq \lambda_{j}^{2} \rightarrow \infty, P_{V} \varphi_{j}=\lambda_{j}^{2} \varphi_{j},\left.\varphi_{j}\right|_{\partial \Omega}=0$, $\left\langle\varphi_{i}, \varphi_{j}\right\rangle_{L^{2}(\Omega)}=\delta_{i j}$. For $j \in-\mathbb{N}^{*}$ we then put

$$
\lambda_{-j}:=-\lambda_{j}<0, \varphi_{-j}:=\varphi_{j}
$$

The solution of (2.48) on $\mathbb{R} \times \Omega$ has an expansion converging in $\mathcal{C}^{\infty}\left(\mathbb{R} ; L^{2}(\Omega)\right)$ :

$$
\begin{aligned}
w(t, x) & =\sum_{j \in \mathbb{Z}^{*}} e^{-i \lambda_{j} t} u_{j}(x), \\
u_{j}(x) & =\frac{1}{2}\left(\left\langle f, \varphi_{j}\right\rangle_{L^{2}(\Omega)}+i \lambda_{j}^{-1}\left\langle g, \varphi_{j}\right\rangle_{L^{2}(\Omega)}\right) \varphi_{j}(x) .
\end{aligned}
$$

A more invariant way to express $u_{j}^{\prime} s$ is given by (2.50) with $R_{V}(\lambda)=\left(P_{V}-\lambda^{2}\right)^{-1}$, the resolvent of the Dirichlet realization of $P_{V}$ on $\Omega$.

\footnotetext{
2 This is expected to be true generically and is claimed in [156]. The proof is incomplete so this seems to be an open problem.
} 
Hence (2.49) is an analogue of the standard expansion (2.52). However, for large times the solution $u(t, x)$ (that is both $u_{j}$ 's and the remainder $E_{A}$ ) are more regular. The proof shows (by applying (2.46) with $j=2$, which is also valid) that

$$
\left\|E_{a}(t)\right\|_{H^{2}\left(B\left(0, R_{1}\right)\right)} \leq C e^{-t a}\left(\|f\|_{H^{1}}+\|g\|_{L^{2}}\right), \quad t>10 R_{1},
$$

and if $V \in \mathcal{C}^{\infty}$ we could replace $H^{2}\left(B\left(0, R_{1}\right)\right)$ in $(2.53)$ by $H^{p}$ for any $p$. That smoothing is visible in Fig. 6. This indicates in a simple case a relation between distribution of resonances and propagation of singularities—see [262], [80, §4.6] and also $[14,16]$ and [99] for some recent applications.

In (2.53) we did not aim at the optimality of the condition $t>10 R_{1}$ : using propagation of singularities it suffices to take $t>2 R_{1}$.

Proof of Theorem 5 We first consider (2.48) with $f \equiv 0$ and $g \in H_{\text {comp. By the }}^{2}$ spectral theorem, the solution of (2.48) can be written as

$$
w(t)=U(t) g:=\frac{\sin t \sqrt{P_{V}}}{\sqrt{P_{V}}}=\int_{0}^{\infty} \frac{\sin t \lambda}{\lambda} d E_{\lambda}(g)
$$

where $d E_{\lambda}$ is the spectral measure, which just as in the case of the free operator $P_{0}$ (2.5), (2.6), can be expressed using Stone's formula [80, Theorem B.8]:

$$
d E_{\lambda}=\frac{1}{\pi i}\left(R_{V}(\lambda)-R_{V}(-\lambda)\right) \lambda d \lambda .
$$

Hence, as in (2.7),

$$
\begin{aligned}
w(t) & =\frac{1}{\pi i} \int_{0}^{\infty} \sin t \lambda\left(R_{V}(\lambda)-R_{V}(-\lambda)\right) g d \lambda \\
& =\frac{1}{2 \pi} \int_{\mathbb{R}} e^{-i t \lambda}\left(R_{V}(\lambda)-R_{V}(-\lambda)\right) g d \lambda .
\end{aligned}
$$

To justify convergence of the integral we use the spectral theorem (see 2.55) which shows that

$$
\left(R_{V}(\lambda)-R_{V}(-\lambda)\right)\left(1+P_{V}\right)=\left(1+\lambda^{2}\right)\left(R_{V}(\lambda)-R_{V}(-\lambda)\right) .
$$

From that we conclude that for $\chi \in \mathcal{C}_{\mathrm{c}}^{\infty}$ equal to 1 on supp $g$,

$$
\begin{aligned}
\chi\left(R_{V}(\lambda)-R_{V}(-\lambda)\right) \chi g & =\chi\left(R_{V}(\lambda)-R_{V}(-\lambda)\right) g \\
& =\chi\left(R_{V}(\lambda)-R_{V}(-\lambda)\right) \chi\left(1+\lambda^{2}\right)^{-1}\left(1+P_{V}\right) g .
\end{aligned}
$$

Theorem 4 and the assumption that there is no zero resonance ${ }^{3}$ shows that

\footnotetext{
3 We do not prove it here but it is a consequence of Rellich's uniqueness theorem that for $V$ real valued $R_{V}(\lambda)$ has no poles in $\mathbb{R} \backslash\{0\}$ —see $[80, \S 3.6]$.
} 


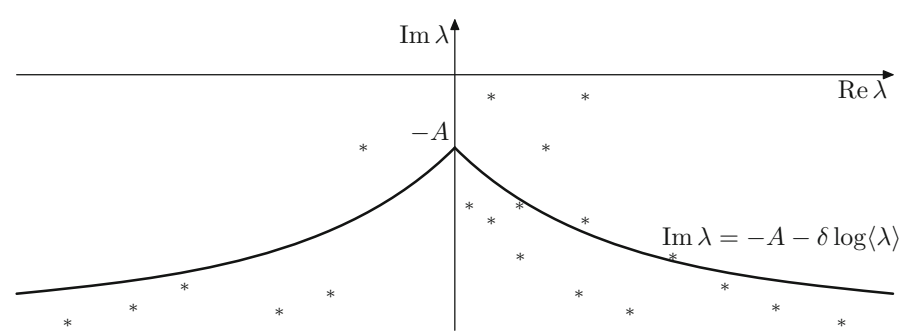

Fig. 16 The contour used to obtain the resonance expansion

$$
\| \chi\left(R_{V}(\lambda)-R_{V}(-\lambda) \chi \|_{L^{2} \rightarrow H^{1}} \leq C, \quad \lambda \in \mathbb{R} .\right.
$$

Hence the integral on the right hand side of (2.56) converges in $H_{\text {loc }}^{1}$.

We now want to deform the contour in (2.56). For that we choose $r$ large enough so that all the resonances with $\operatorname{Im} \lambda>-a-\delta \log (1+|\lambda|)$ are contained in $|\lambda| \leq r$. If we choose $\delta<\frac{1}{2 R_{0}}$ that is possible thanks to Theorem 4 .

We now deform the contour of integration using the following contours:

$$
\begin{aligned}
\Gamma & :=\{\lambda-i(a+\varepsilon+\delta \log (1+|\lambda|)): \lambda \in \mathbb{R}\}, \\
\gamma_{r}^{ \pm}: & =\{ \pm r-i t: 0 \leq t \leq a+\varepsilon+\delta \log (1+r)\}, \quad \gamma_{r}:=\gamma_{r}^{+} \cup \gamma_{r}^{-}, \\
\Gamma_{r} & :=\Gamma \cap\{|\lambda| \leq r\}, \quad \gamma_{r}^{\infty}:=(-\infty,-r) \cup(r, \infty) .
\end{aligned}
$$

Here we choose $\varepsilon$ and so that there are no resonances on $\Gamma$. We also put

$$
\Omega_{a}:=\{\lambda: \operatorname{Im} \lambda \geq-a-\varepsilon-\delta \log (1+|\lambda|)\}
$$

and define

$$
\Pi_{a}(t):=i \sum_{\lambda \in \Omega_{a}} \operatorname{Res}_{\lambda=\lambda_{j}}\left(\chi R_{V}(\lambda) \chi e^{-i \lambda t}\right) .
$$

In this notation the residue theorem shows that $U(t)$ defined in (2.54) is given by

$$
\chi U(t) g=\Pi_{A}(t) g+E_{\Gamma_{r}}(t)+E_{\gamma_{r}}(t)+E_{\gamma_{r}^{\infty}}(t)
$$

where (with natural orientations—-see Fig. 16)

$$
\left.E_{\gamma}(t):=\frac{1}{2 \pi} \int_{\gamma} e^{-i t \lambda} \chi\left(R_{V}(\lambda)\right)-R_{V}(-\lambda)\right) \chi g d \lambda .
$$

Using (2.46) and (2.57) we obtain

$$
\left\|E_{\gamma_{r}^{\infty}}(t) g\right\|_{H^{1}} \leq C \int_{r}^{\infty}\left(1+|\lambda|^{2}\right)^{-1}\|g\|_{H^{2}} \leq \frac{C}{r}\|g\|_{H^{2}} \rightarrow 0, \quad r \rightarrow \infty,
$$


and

$$
\left\|E_{\gamma_{r}}(t) g\right\|_{H^{1}} \leq \frac{C(1+r)^{4 R_{1} \delta}}{1+r^{2}}\|g\|_{H^{2}} \rightarrow 0, \quad r \rightarrow \infty \text { if } \delta<\frac{1}{2 R_{1}} .
$$

Returning to (2.59) we see that

$$
\chi U(t) \chi g=\Pi_{A}(t) g+E_{\Gamma}(t) g, g \in H^{2},
$$

where $E_{\Gamma}$ is defined using (2.60) with $\Gamma$ given in (2.58).

We now show that when $t$ is large enough,

$$
\left\|E_{\Gamma}(t) g\right\|_{H^{1}} \leq C e^{-t a}\|g\|_{L^{2}} .
$$

For that we use (2.46) with $j=1$ for $|\lambda|>R$, and the assumption that there are no poles of $R_{V}(\lambda)$ near $\Gamma$. Thus we obtain:

$$
\begin{aligned}
\left\|E_{\Gamma}(t) g\right\|_{H^{1}} & \leq C e^{-a t} \int_{\mathbb{R}} e^{-t \delta \log (1+|\lambda|)} e^{\delta 2 R_{1} \log (1+|\lambda|)}\|g\|_{L^{2}} d \lambda \\
& \leq C e^{-a t} \int_{\mathbb{R}}(1+|\lambda|)^{-\delta\left(t-2 R_{1}\right)}\|g\|_{L^{2}} d \lambda \\
& \leq C_{\varepsilon} e^{-a t}\|g\|_{L^{2}}, \quad t>2 R_{1}+1 / \delta+\varepsilon .
\end{aligned}
$$

Since $H^{1}$ is dense in $L^{2}$ the decomposition (2.61) is valid for $g \in L^{2}$ proving theorem for $f=0$, once $t$ is large enough. But for bounded $t,\|w(t)\|_{H^{1}} \leq C\|g\|_{L^{2}}$. The result follows by noting that $\chi E_{a}(t)=E_{\Gamma}(t) g$.

The case of arbitrary $f \in H_{\mathrm{comp}}^{1}$ and $g \equiv 0$ follows by replacing $\sin t \lambda / \lambda$ by $\cos t \lambda$ in the formula for $w(t, x)$.

The expansion of waves provides an (admittedly) weak approximation to correlations (see Fig. 3) related to the Breit-Wigner approximation. Suppose $g \in$ $L^{2}\left(B\left(0, R_{1}\right)\right), h \in H^{-1}\left(B\left(0, R_{0}\right)\right)$ and put $U(t):=\sin t \sqrt{P}_{V} / \sqrt{P}_{V}$. We define the following correlation function

$$
\rho_{g, h}(t):=\int_{\mathbb{R}^{3}} U(t) g(x) \overline{h(x)} d x,
$$

and its power spectrum:

$$
\hat{\rho}_{g, h}(\lambda)=\int_{0}^{\infty} \rho_{g, h}(t) e^{i \lambda t} d t
$$

A formal application of the expansion (2.56) suggests

$$
\hat{\rho}_{g, h}(\lambda) \sim \sum_{\operatorname{Im} \lambda_{j}>-a} \frac{\operatorname{Res}_{\lambda=\lambda_{j}}\left\langle R_{V}(\lambda) g, h\right\rangle}{\lambda-\lambda_{j}}
$$


but it is difficult to give useful remainder estimates in general. Expansion (2.65) is just an expansion of a meromorphic function with simple poles (as assumed here) following an analogue of (2.11).

For Breit-Wigner type formula near a single resonance in the semiclassical limit see Gérard-Martinez [105] and Gérard-Martinez-Robert [106] and for high energy results and results for clouds of resonances, Petkov-Zworski [211,212] and NakamuraStefanov-Zworski [194].

We finally comment on expansions on the case of the Schrödinger equation. In that case the dispersive nature of the equation makes it harder to justify resonance expansions. It can be done when a semiclassical parameter is present, see Burq-Zworski [40] and Nakamura-Stefanov-Zworski [194], or when one considers a localized resonance-see Merkli-Sigal [185], Soffer-Weinstein [253] and references given there.

\subsection{Complex scaling in dimension one}

In Sect. 2.2 we established meromorphic continuation using analytic Fredholm theory. That allowed us to obtain general bounds on the number of resonances and to justify resonance expansions. To obtain more refined results relating geometry to the distribution of resonances, as indicated in Sect. 1.3, we would like to use spectral theory of partial differential equations. And for that we would like to have a differential operator whose eigenvalues would be given by resonances. That operator should also have suitable Fredholm properties. We would call this an effective meromorphic continuation.

The method of complex scaling produces a natural family of non-self-adjoint operators whose discrete spectrum consists of resonances. It originated in the work of Aguilar-Combes [3], Balslev-Combes [10] and was developed by Simon [239], Hunziker [138], Helffer-Sjöstrand [125], Hislop-Sigal [133] and other authors. For very general compactly supported "black box" perturbations and for large angles of scaling it was studied in [245] while the case of long range black box perturbations was worked out in [241]. The method has been extensively used in computational chemistry-see Reinhardt [222] for a review. As the method of perfectly matched layers it reappeared in numerical analysis-see Berenger [19].

In this section we present the simplest case of the method by working in one dimension. ${ }^{4}$ The idea is to consider $D_{x}^{2}$ as a restriction of the complex second derivative $D_{z}^{2}$ to the real axis thought of as a contour in $\mathbb{C}$. This contour is then deformed away from the support of $V$ so that $P=D_{x}^{2}+V(x)$ can be restricted to it. This provides ellipticity at infinity at the price of losing self-adjointness.

Before proceeding we need to discuss the definition of resonances in dimension one. In that case the free resolvent, $R_{0}(\lambda)=\left(P_{0}-\lambda^{2}\right)^{-1}, P_{0}=-\partial_{x}^{2}$, has a very simple Schwartz kernel,

\footnotetext{
4 Our presentation is based on [245, §2] and on an upublished note by Kiril Datchev http://www.math. purdue.edu/ kdatchev/res.ps.
} 


$$
R_{0}(\lambda, x, y)=\frac{i}{2 \lambda} e^{i \lambda|x-y|}
$$

which means that as an operator $R_{0}(\lambda): L_{\text {comp }}^{2}(\mathbb{R}) \rightarrow L_{\text {loc }}^{2}(\mathbb{R})$, the resolvent continues to a meromorphic family with a single pole at $\lambda=0 .^{5}$

The approach of Sect. 2.2 carries through without essential modifications and in particular the characterization of resonant states (2.24) applies. Since for $\lambda \neq 0$,

$$
u(x)=R_{0}(\lambda) f, \quad f \in L_{\text {comp }}^{2} \Longleftrightarrow u(x)=a_{ \pm} e^{ \pm i \lambda x}, \quad \pm x \gg 1, u \in H_{\text {loc }}^{2}(\mathbb{R}),
$$

we have the following characterization of scattering resonances

$$
\begin{aligned}
& \lambda \neq 0 \text { is a scattering resonance of } P_{V}=-\partial_{x}^{2}+V(x), V \in L_{\text {comp }}^{\infty}(\mathbb{R}) \\
& \quad \text { 至 } \\
& \quad \exists u \in H_{\mathrm{loc}}^{2}(\mathbb{R}), \quad\left(P_{V}-\lambda^{2}\right) u=0, \quad u(x)=a_{ \pm} e^{ \pm i \lambda x} \neq 0, \quad \pm x \gg 1,
\end{aligned}
$$

For reasons of simplicity we will not consider multiplicities. The only possibility here is having algebraic multiplicities since solutions satisfying the condition in (2.66) are unique up to a multiplicative constant.

We now construct an operator which has resonances as its discrete spectrum. For that let $\Gamma \subset \mathbb{C}$ be a $C^{1}$ simple curve. We define differentiation and integration of functions mapping $\Gamma$ to $\mathbb{C}$ as follows. Let $\gamma(t)$ be a parametrization $\mathbb{R} \rightarrow \Gamma$, and let $f \in C^{1}(\Gamma)$ in the sense that $f \circ \gamma \in C^{1}(\mathbb{R})$. We define

$$
\partial_{z, \Gamma} f\left(z_{0}\right)=\gamma^{\prime}\left(t_{0}\right)^{-1} \partial_{t}(f \circ \gamma)\left(t_{0}\right), \quad \gamma\left(t_{0}\right)=z_{0},
$$

where the inverse and the multiplication are in the sense of complex numbers.

The chain rule shows that $\partial_{z}^{\Gamma} f\left(z_{0}\right)$ is independent of parametrization and that for $F$ holomorphic near $\Gamma$,

$$
\left.\partial_{z, \Gamma} F\right|_{\Gamma}=\left.\partial_{z} F\right|_{\Gamma}
$$

where $\partial_{z}=\frac{1}{2}\left(\partial_{x}-i \partial_{y}\right)($ see $[80, \S 4.6])$.

We make the following assumption on the behaviour of $\Gamma$ at infinity:

$$
\exists 0<\theta<\pi, z_{ \pm} \in \mathbb{C}, K \Subset \mathbb{C}, \quad \Gamma \backslash K=\bigcup_{ \pm}\left(z_{ \pm} \pm e^{i \theta}(0, \infty)\right) \backslash K .
$$

An example of $\Gamma$ is shown in Fig. 17. One can consider more general behaviour at infinity such as shown in Fig. 18 where $\Gamma=\{x+i g(x): x \in \mathbb{R}\}$ for a specific $g$.

Given a potential $V \in L_{\text {comp }}^{\infty}(\mathbb{R} ; \mathbb{C})$ we further assume that

$$
\Gamma \cap \mathbb{R} \supset[-L, L], \quad \operatorname{supp} V \subset(-L, L) .
$$

\footnotetext{
5 This is consistent with the expansion (1.2) for the free wave equation: the single pole is "responsible" for the failure of the sharp Huyghens principle in that case.
} 


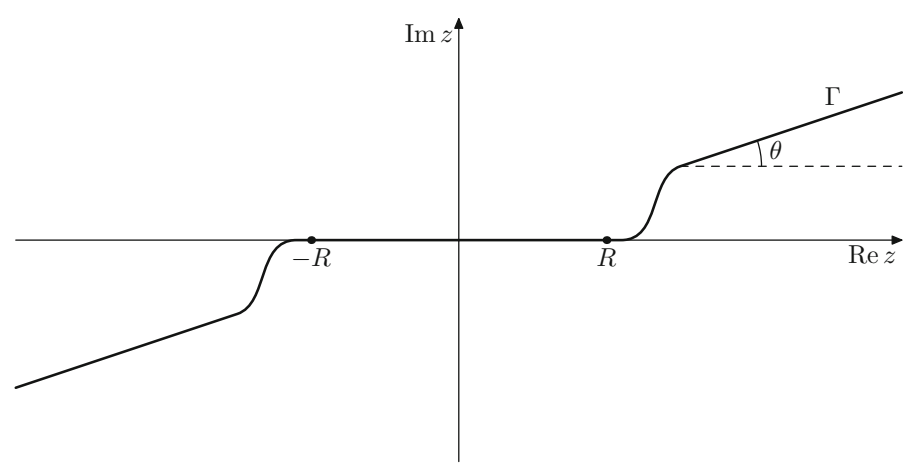

Fig. 17 Curve $\Gamma$ used in complex scaling. The curve is given by $x \mapsto x+i g(x)$ for a $C^{\infty}$ function $g$ satisfying $g(x)=0$ for $-R \leq x \leq R$ and $g(x)=x \tan \theta$ for $|x|$ sufficiently large, where $\theta$ is a given constant

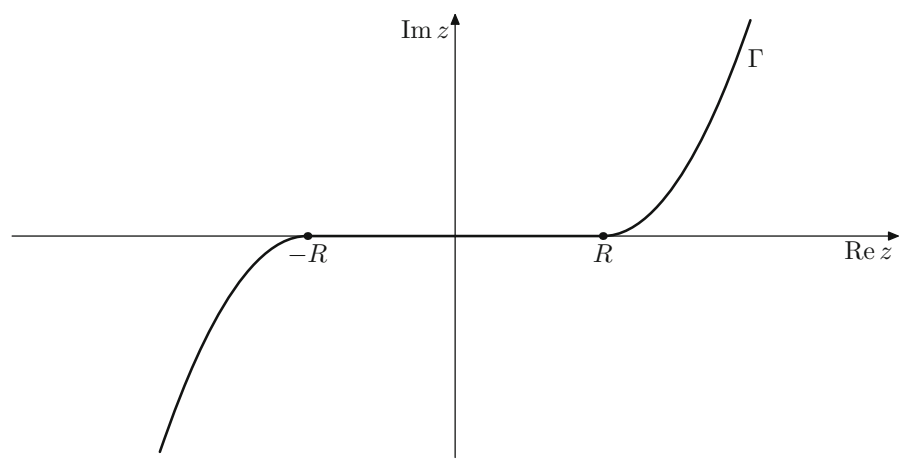

Fig. 18 Curve $\Gamma$ used in PML computations—-see [20] and references given there. A typical curve is given by a function $\mathbb{R} \ni x \mapsto x+i g(x)$ where $g(x)=-|x+R|^{\alpha}$ for $x<-R, g(x)=0$ for $-R \leq x \leq R$, and $g(x)=(x-L)^{\alpha}$ for $x>R$, where $\alpha>1$

The potential $V$ is then a well defined function on $\Gamma$, so that putting

$$
P_{V, \Gamma}:=-\partial_{z, \Gamma}^{2}+V(z)
$$

makes sense.

We now want to relate eigenvalues of $P_{V, \Gamma}$ to the resonances, that is to $\lambda$ 's for which (2.66) holds. For that let us write $\Gamma$ as a disjoint union of connected components:

$$
\Gamma=\Gamma_{-} \cup[-L, L] \cup \Gamma_{+}
$$

where $\operatorname{Im} z \rightarrow \pm \infty$ on $\Gamma_{ \pm}$. We then observe that

$$
\left(P_{V, \Gamma}-\lambda^{2}\right) u_{\Gamma} \Longrightarrow u_{\Gamma}(z)=a_{ \pm} e^{ \pm i \lambda z}+b_{ \pm} e^{\mp i \lambda z}, \quad z \in \Gamma_{ \pm} .
$$


That is because on $\Gamma_{ \pm}$we are away from the support of the potential and the unique solutions to our differential equations have to come from holomorphic solutions to

$$
\left(-\partial_{z}^{2}-\lambda^{2}\right) U_{ \pm}=0,\left.\quad u_{\Gamma}\right|_{\Gamma_{ \pm}}=\left.U_{ \pm}\right|_{\Gamma_{ \pm}}
$$

Condition (2.67) shows that

$$
\begin{aligned}
& \operatorname{Re}\left( \pm\left. i \lambda z\right|_{\Gamma_{ \pm}}\right)=-\sin (\theta+\arg \lambda)|\lambda||z|+\mathcal{O}(1)<-\varepsilon|\lambda||z|+\mathcal{O}(1), \\
& \operatorname{Re}\left(\left.\mp i \lambda z\right|_{\Gamma_{ \pm}}\right)=\sin (\theta+\arg \lambda)|\lambda||z|+\mathcal{O}(1)>\varepsilon|\lambda||z|+\mathcal{O}(1) .
\end{aligned}
$$

Hence $u_{\Gamma} \in L^{2}(\Gamma)$ if and only if $b_{ \pm}=0$ in (2.70). But then

$$
u(x):= \begin{cases}\left.U_{+}\right|_{[L, \infty)}(x), & x>L, \\ u_{\Gamma}(x), & x \in[-L, L], \\ \left.U_{-}\right|_{(-\infty,-L]}(x), & x<-L,\end{cases}
$$

satisfies the condition in (2.66) and hence $\lambda$ is a resonance.

This argument can be reversed and consequently we basically proved [80, Theorem 2.20]:

Theorem 6 (Complex scaling in dimension one) Suppose that $\Gamma$ satisfies (2.67) and $P_{V, \Gamma}$ is defined by $(2.69)$ with $V \in L_{\text {comp }}^{\infty}(\mathbb{R} ; \mathbb{C})$. Define

$$
\Lambda_{\Gamma}:=\left\{\lambda \in \mathbb{C} \backslash \overline{\mathbb{R}}_{-}:-\theta<\arg \lambda<\pi-\theta\right\}
$$

where $\arg : \overline{\mathbb{R}}_{-} \rightarrow(-\pi, \pi)$.

For $\lambda \in \Lambda_{\Gamma}$,

$$
P_{V, \Gamma}-\lambda^{2}: H^{2}(\Gamma) \rightarrow L^{2}(\Gamma),
$$

is a Fredholm operator and the spectrum of $P_{\Gamma, V}$ in $\Lambda_{\Gamma}$ is discrete.

Moreover, the eigenvalues of $P_{V, \Gamma}$ in $\Lambda_{\Gamma}$ coincide with the resonances of $P_{V}$ there, and

$$
m(\lambda)=\frac{1}{2 \pi i} \operatorname{tr}_{L^{2}(\Gamma)} \oint_{\lambda}\left(\zeta^{2}-P_{V, \Gamma}\right)^{-1} 2 \zeta d \zeta, \quad \lambda \in \Lambda_{\Gamma},
$$

where $m(\lambda)$ is the multiplicity of the resonance at $\lambda$ (see Definition 1) and the integral is over a sufficiently small positively oriented circle enclosing $\lambda$.

Interpretation. We first remark that

$$
\Pi_{\lambda, \Gamma}=\frac{1}{2 \pi i} \oint_{\lambda}\left(\zeta^{2}-P_{V, \Gamma}\right)^{-1} 2 \zeta d \zeta: L^{2}(\Gamma) \rightarrow L^{2}(\Gamma),
$$

is a projection. Hence, the method of complex scaling identifies the multiplicity of a resonance with a trace of a (non-orthogonal) projection. We gain the advantage of being able to use methods of spectral theory, albeit in the murkier non-normal setting. The resonant states, that is the outgoing solutions to $\left(P-\lambda^{2}\right) u$, are restrictions to $\mathbb{R}$ of 
functions which continue holomorphically to fuctions with $L^{2}$ restrictions to $\Gamma$. Since have dealt only with compactly supported potentials our countours $\Gamma$ had to coincide with $\mathbb{R}$ near the support of $V$. The method generalizes to the case of potentials which are analytic and decaying in conic neighbourhoods of $\pm(L, \infty)$. Generalizations to higher dimensions are based on the same principles but the treatment is no longer as explicit.

\subsection{Other results and open problems}

For $V \in \mathcal{C}_{\mathrm{c}}^{\infty}\left(\mathbb{R}^{n}\right)$, real valued, existence of infinitely many resonances was proved by Melrose [184] for $n=3$ and by Sá Barreto-Zworski [230] for all odd $n$ (including for any superexponentially decaying potentials). The surprising fact that there exist complex valued potentials in odd dimensions greater than or equal to three that have no resonances at all was discovered by Christiansen [46].

Quantitative statements about the counting function, $N_{V}(r)$, of resonances in $\{|\lambda| \leq$ $r$ \} were obtained by Christiansen [45] and Sá Barreto [229]:

$$
\limsup _{r \rightarrow \infty} \frac{N_{V}(r)}{r}>0, \quad V \in \mathcal{C}_{\mathrm{c}}^{\infty}\left(\mathbb{R}^{n} ; \mathbb{R}\right), \quad V \neq 0 .
$$

For potentials generic in $\mathcal{C}_{\mathrm{c}}^{\infty}\left(\mathbb{R}^{n} ; \mathbb{F}\right)$ or $L_{\mathrm{c}}^{\infty}\left(\mathbb{R}^{n} ; \mathbb{F}\right), \mathbb{F}=\mathbb{R}$ or $\mathbb{C}$, Christiansen and Hislop [47] proved a stronger statement

$$
\limsup _{r \rightarrow \infty} \frac{\log N_{V}(r)}{\log r}=n
$$

The limit (2.74) means that the upper bound $N_{V}(r) \leq C r^{n}$ in Theorem 3 is optimal for generic complex or real valued potentials. The only case of asymptotics $\sim r^{n}$ for non-radial potentials was provided by Dinh and $\mathrm{Vu}$ [58] who proved that potentials in a large subset of $L^{\infty}(B(0,1))$ have resonances satisfying the Weyl law (2.28). The proofs of these results use techniques from several complex variables.

Recently Smith-Zworski [252] showed that any real valued

$$
V \in H^{\frac{n-3}{2}}\left(\mathbb{R}^{n}\right) \cap L_{\text {comp }}^{\infty}\left(\mathbb{R}^{n}\right),
$$

has infinitely many resonances and any $V \in L_{\text {comp }}^{\infty}\left(\mathbb{R}^{n}\right)$ has some resonances ( $n$ odd). It is still a (ridiculous) open question if every real bounded potential $(\neq 0)$ has infinitely many resonances.

The most frustrating open problem is existence of an optimal lower bound. It is not clear if we can expect an asymptotic formula.

Conjecture 1 Suppose $V \in L_{\text {comp }}^{\infty}\left(\mathbb{R}^{n}\right), n$ odd, is real-valued and non-zero. Then there exists $c>0$ such that

$$
N_{V}(r) \geq c r^{n}
$$


where the counting function $N_{V}(r)$ is defined in (2.25).

All of the above questions can be asked in even dimensions and for obstacle problems in which case the bound (2.26) was established by Melrose [182] for odd dimensions and by Vodev [266,267] in even dimension. A remarkable recent advance is due to Christiansen [48] who proved that for any obstacle in even dimensions we always have $r^{n}$ growth for the number of resonances-see that paper for other references concerning lower bounds in even dimension.

Existence of resonances can be considered as a primitive inverse problem: a potential with no resonances is identically zero. In one dimension or in the radial case finer inverse results have been obtained: see Korotyaev [160,161], Brown-KnowlesWeikard [36], Bledsoe-Weikard [18], Datchev-Hezari [55] and references given there.

Another recent development in the study of resonances for compactly supported potentials concerns highly oscillatory potentials $V(x)=W(x, x / \varepsilon)$ where $W: \mathbb{R}^{n} \times$ $\mathbb{R}^{n} / \mathbb{Z}^{n} \rightarrow \mathbb{R}$ (or $\mathbb{C}$ ) is compactly supported in the first set of variables. Precise results for $n=1$ were obtained by Duchêne-Vukićević-Weinstein [63]: resonances are close to resonances of $W_{0}(x):=\int_{\mathbb{R}^{n} / \mathbb{Z}^{n}} W(x, y) d y$, and the difference is given by $\varepsilon^{4} \alpha+\mathcal{O}\left(\varepsilon^{5}\right)$ where, in the spirit of homogenization theory, $\alpha$ can be computed using an effective potential. In a remarkable follow-up Drouot [61] generalized this result to all odd dimensions and obtained a full asymptotic expansion in powers of $\varepsilon$.

\section{Some recent developments}

We will now describe some recent developments in meromorphic continuation, resonance free regions, resonance counting and resonance expansions for semiclassical operators $-h^{2} \Delta_{g}+V$ on Riemannian manifolds with Euclidean and non-Euclidean infinities. We will also formulate some conjectures: some quite realistic (even numbered) and some perhaps less so (odd numbered). ${ }^{6}$

Before doing it let us mention some interesting topics which lie beyond the scope of this survey. We will discuss hyperbolic trapped sets but not homoclinic trapped sets. Although not stable under perturbations these trapped sets occur in many situations each with its own interesting structure in distribution of resonances. An impressively precise study of this has been made by Bony-Fujiie-Ramond-Zerzeri [28]. In our survey of counting results we will concentrate on fractal Weyl laws and we refer to Borthwick-Guillarmou [32] for recent results on resonance counting in geometric settings. In the semiclassical Euclidean setting Sjöstrand [244] obtained precise asymptotic counting results in the case of random potentials. The introduction of randomness should be a beginning of a new development in the subject. We also do not discuss the important case of resonances for magnetic Schrödinger operators and refer to Alexandrova-Tamura [4], Bony-Bruneau-Raikov, [26] and Tamura [258,259] for

\footnotetext{
6 Small prizes are offered by the author for the first proofs of the conjectures within five years of the publication of this survey: a dinner in a $\varepsilon^{2} \varepsilon^{3}$ restaurant for an even numbered conjecture and in a $\varepsilon_{3} \varepsilon_{3} \varepsilon_{3}$ restaurant, for an odd numbered one.
} 
some recent results and pointers to the literature. We do not address issues around threshold resonances (see Jensen-Nenciu [150] and references given there) and their importance in dispersive estimates (see the survey by Schlag [233]) and for non-linear equations (for an example of a linearly counterintuitive phenomenon, see [135, Figure 6]). Finally, for the role that shape resonances have had in the study of blow-up phenomena we defer to Perelman [208] and Holmer-Liu [134] and to the references given there.

The section is organized as follows: in Sect. 3.1 we review Vasy's method for defining resonances for asymptotically hyperbolic manifolds. In Sect. 3.2 we first review general results on resonance free regions and then describe the case of hyperbolic (in the dynamical sense) trapped sets. As applications of results in Sects. 3.1 and 3.2 we discuss expansions of waves in black hole backgrounds in Sect. 3.3. The last Sect. 3.4 is devoted to mathematical study of fractal Weyl laws with some references to the growing physics literature on that subject.

\subsection{Meromorphic continuation in geometric scattering}

In Sect. 2.2 we showed how to continue the resolvent meromorphically across the spectrum using analytic Fredholm theory. In Sect. 2.6 we presented the method of complex scaling which provides an effective meromorphic continuation in the sense that resonances are identified with eigenvalues of non-self-adjoint Fredholm operators. As one can see already in the one dimensional presentation that method is closely tied to the structure of the operator near infinity.

With motivation coming from general relativity in physics (see Sect. 3.3) and from analysis on locally symmetric spaces it is natural to consider different structures near infinity. Here we will discuss complete asymptotically hyperbolic Riemannian manifolds modeled on the hyperbolic space near infinity. For more general hyperbolic ends see Guillarmou-Mazzeo [119] where meromorphy of the resolvent was established using analytic Fredholm theory without providing an effective meromorphic continuation as defined above. We also remark that complex scaling method is possible in the case of manifolds with cusps. For a subtle application of that see a recent paper by Datchev [52] where existence of arbitrarily wide resonance free strips for negative curvature perturbations of $\langle z \mapsto z+1\rangle \backslash \mathbb{H}^{2}$ is established. For a recent analysis of a higher rank symmetric spaces and references to earlier works see Mazzeo-Vasy [177,178] and Hilgert-Pasquale-Przebinda [126].

A basic example of our class of manifolds is given by the hyperbolic space $\mathbb{H}^{n}$, which can be viewed as the open unit ball in $\mathbb{R}^{n}$ with the metric

$$
g=4 \frac{d w^{2}}{\left(1-|w|^{2}\right)^{2}}, \quad w \in B_{\mathbb{R}^{n}}(0,1)
$$

A more interesting family of examples is provided by convex co-compact hyperbolic surfaces, which are complete two-dimensional Riemannian manifolds of constant sectional curvature -1 whose infinite ends are funnels, that is they have the form 

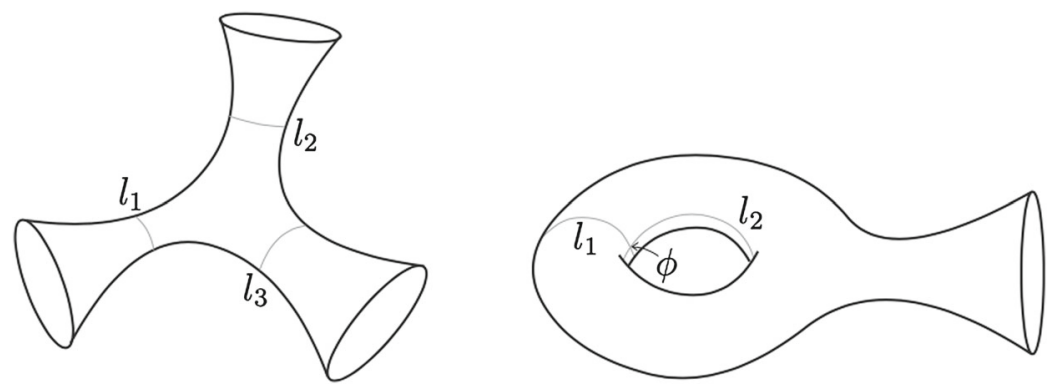

Fig. 19 Left surfaces $X\left(\ell_{1}, \ell_{2}, \ell_{3}\right)$ with three funnels determined by lengths of the geodesic boundaries of the funnels (3.2) — see [31, $\$ 16.2 .1]$. Right funneled tori $Y\left(\ell_{1}, \ell_{2}, \varphi\right)$ parametrized by the length of the two geodesics generating homotopy group of the torus and the angle between. The left example has infinity with three components; the right one with one component

$$
[0, \infty)_{v} \times \mathbb{S}_{\theta}^{1}, \quad \mathbb{S}^{1}=\mathbb{R} / \ell \mathbb{Z}, \ell>0 ; \quad g=d v^{2}+\cosh ^{2} v d \theta^{2}
$$

Convex co-compact hyperbolic surfaces can be viewed as the quotients of $\mathbb{H}^{2}$ by certain discrete subgroups of its isometry group $\operatorname{PSL}(2 ; \mathbb{R})[31]$, and have profound applications in algebra and number theory-see for instance Bourgain-GamburdSarnak [35]. Furthermore, they give fundamental examples of hyperbolic trapped sets and are a model object to study the effects of hyperbolic trapping on distribution of resonances. Examples which will be discussed in Sects. 3.2, 3.4 are shown in Fig. 19.

The examples above are generalized as even asymptotically hyperbolic manifolds. To define that class of manifolds, suppose that $\bar{M}$ is a compact manifold with boundary $\partial M \neq \emptyset$ of dimension $n+1$. We denote by $M$ the interior of $\bar{M}$. The Riemannian manifold $(M, g)$ is even asymptotically hyperbolic if there exist functions $y^{\prime} \in \overline{\mathcal{C}}^{\infty}(M ; \partial M)$ and $y_{1} \in \overline{\mathcal{C}}^{\infty}(M ;(0,2))^{7},\left.y_{1}\right|_{\partial M}=0,\left.d y_{1}\right|_{\partial M} \neq 0$, such that

$$
\bar{M} \supset y_{1}^{-1}([0,1)) \ni m \mapsto\left(y_{1}(m), y^{\prime}(m)\right) \in[0,1) \times \partial M
$$

is a diffeomorphism, and near $\partial M$ the metric has the form,

$$
\left.g\right|_{y_{1} \leq \varepsilon}=\frac{d y_{1}^{2}+h\left(y_{1}^{2}\right)}{y_{1}^{2}},
$$

where $[0,1) \ni t \mapsto h(t)$, is a smooth family of Riemannian metrics on $\partial M$. For the discussion of invariance of this definition and of its geometric meaning we refer to $[80, \S 5.1]$ and $[115, \S 2]$. Here we will point out how it fits with the two examples. For (3.1) we have

$$
g=4 \frac{d w^{2}}{\left(1-|w|^{2}\right)^{2}}=\frac{d y_{1}^{2}+h\left(y_{1}^{2}\right)}{y_{1}^{2}},
$$

\footnotetext{
7 Here we follow the notation of [137, Appendix B] where $\overline{\mathcal{C}}^{\infty}(M ; V)$ denotes functions $M \rightarrow V$, which are smoothly extendable across $\partial M$ and $\dot{\mathcal{C}}^{\infty}(\bar{M} ; V)$ functions which are extendable to smooth functions supported in $\bar{M}$.
} 


$$
y_{1}:=\frac{2(1-|w|)}{1+|w|} \in[0,2), \quad y^{\prime}:=\frac{w}{|w|} \in \mathbb{S}^{n-1}, \quad h(t)=(1-t)^{2} h_{0}\left(y^{\prime}, d y^{\prime}\right)
$$

where $h_{0}$ is the standard metric on the unit sphere $\mathbb{S}^{n-1}$. In the case of (3.2), we have a coordinate system $y$ on $\{v>0\}$ :

$$
\begin{gathered}
g=d v^{2}+\cosh ^{2} v d \theta^{2}=\frac{d y_{1}^{2}+h\left(y_{1}^{2}\right)}{y_{1}^{2}}, \\
y_{1}=2 e^{-v} \in[0,2), \quad y_{2}=\theta \in \mathbb{S}^{1}, \quad h(t)=(1+t)^{2} d y_{2}^{2} .
\end{gathered}
$$

Let $-\Delta_{g} \geq 0$ be the Laplace-Beltrami operator for the metric $g$. Since $-\Delta_{g}$ is a self-adjoint operator, the spectrum is contained in $[0, \infty)$ the operator $-\Delta_{g}-\lambda^{2}-\left(\frac{n}{2}\right)^{2}$ is invertible on $H^{2}\left(M, d \operatorname{vol}_{g}\right)$ for $\operatorname{Im} \lambda>\frac{n}{2}$. Hence we can define

$$
R(\lambda):=\left(-\Delta_{g}-\lambda^{2}-\frac{n^{2}}{4}\right)^{-1}: L^{2}\left(M, d \operatorname{vol}_{g}\right) \rightarrow H^{2}\left(M, d \operatorname{vol}_{g}\right), \quad \operatorname{Im} \lambda>\frac{n}{2}
$$

It turns out that $R(\lambda): L^{2} \rightarrow H^{2}$ is meromorphic for $\operatorname{Im} \lambda>0$ : the poles correspond to $L^{2}$ eigenvalues of $-\Delta_{g}$ and hence lie in $i\left(0, \frac{n}{2}\right)$. A closely related standard fact is that the continuous spectrum of $-\Delta_{g}$ is equal to $\left[\left(\frac{n}{2}\right)^{2}, \infty\right)$. This explains our "shifted" convention in defining $R(\lambda)$.

Elliptic regularity shows that $R(\zeta): \dot{\mathcal{C}}^{\infty}(M) \rightarrow \mathcal{C}^{\infty}(M), \operatorname{Im} \lambda>n / 2$. Hence is natural to consider meromorphic continuation of

$$
R(\lambda): \dot{\mathcal{C}}^{\infty}(M) \longrightarrow \mathcal{C}^{\infty}(M), \lambda \in \mathbb{C}
$$

That meromorphy was first established for any asymptotically hyperbolic metric (that is a metric of the form (3.4) but with $\left.h=h\left(y_{1}\right)\right)$ by Mazzeo-Melrose [176]. Other early contributions were made by Agmon [2], Fay [90], Lax-Phillips [163], Mandouvalos [172], Patterson [205-207] and Perry [209]. Guillarmou [115] showed that the evenness condition was needed for a global meromorphic continuation and clarified the construction given in [176].

All these arguments relied on analytic Fredholm theory (2.17) and did not provide an effective continuation in the sense Sect. 2.6. A recent breakthrough due to Vasy [263] provided such effective continuation by expressing $R(\lambda)$ using $P(\lambda)^{-1}$ where

$$
\lambda \mapsto P(\lambda) \text { is a holomorphic family of differential operators. }
$$

Hence, microlocal methods can now be used to prove results which were not available before, for instance existence of resonance free strips for non-trapping metrics [264]. Other applications in the theory of resonances will be presented in Sects. 3.2, 3.4. Roughly speaking, thanks to Vasy's method we can now concentrate on the interaction region where interesting dynamical phenomena occur and treat infinity as a black box, almost in the same way as complex scaling allowed us in the Euclidean case. (See Wunsch-Zworski [273] for a class of asymptotically Euclidean manifolds to which the 
method of complex scaling also applies.) We also mention some applications not covered by this survey: a quantitative version of Hawking radiation by Drouot [61], exponential decay of waves in the Kerr-de Sitter case and the description of quasi-normal modes for perturbations of Kerr-de Sitter black holes by Dyatlov [65,68], rigorous definition of quasi-normal modes for Kerr-Anti de Sitter black holes ${ }^{8}$ by Gannot [101]. The construction of the Fredholm family also plays a role in the study of linear and non-linear scattering problems - see the works of Baskin-Vasy-Wunsch [15], HintzVasy $[129,130]$ and references given there. In particular, it is important in the recent proof of nonlinear stability of Kerr-de Sitter black holes by Hintz and Vasy [131].

We will follow the presentation of [282] and for simplicity will not consider the semiclassical case. That of course is essential for applications and can be found in a textbook presentation of [80, Chapter 5].

Let $y^{\prime} \in \partial M$ denote the variable on $\partial M$. Then (3.4) implies that near $\partial M$, the Laplacian has the form

$$
\begin{aligned}
-\Delta_{g} & =\left(y_{1} D_{y_{1}}\right)^{2}+i\left(n+y_{1}^{2} \gamma\left(y_{1}^{2}, y^{\prime}\right)\right) y_{1} D_{y_{1}}-y_{1}^{2} \Delta_{h\left(y_{1}^{2}\right)}, \\
\gamma\left(t, y^{\prime}\right) & :=-\partial_{t} \bar{h}(t) / \bar{h}(t), \quad \bar{h}(t):=\operatorname{det} h(t), \quad D:=\frac{1}{i} \partial .
\end{aligned}
$$

Here $\Delta_{h\left(y_{1}^{2}\right)}$ is the Laplacian for the family of metrics on $\partial M$ depending smoothly on $y_{1}^{2}$ and $\gamma \in \mathcal{C}^{\infty}([0,1] \times \partial M)$. (The logarithmic derivative defining $\gamma$ is independent of of the density on $\partial M$ needed to define the determinant $\bar{h}$.)

As established in [176] (see [282, §6] for a direct argument) the unique $L^{2}$ solution, $u$, to $\left(-\Delta_{g}-\lambda^{2}-\left(\frac{n}{2}\right)^{2}\right) u=f \in \dot{\mathcal{C}}^{\infty}(M), \operatorname{Im} \lambda>0$, satisfies

$$
u=y_{1}^{-i \lambda+\frac{n}{2}} \overline{\mathcal{C}}^{\infty}(M) \quad \text { and }\left.\quad y_{1}^{i \lambda-\frac{n}{2}} u\right|_{y_{1}<1}=F\left(y_{1}^{2}, y^{\prime}\right), \quad F \in \overline{\mathcal{C}}^{\infty}([0,1] \times \partial M) .
$$

This suggests two things:

- in order to reduce the investigation to the study of regularity we should conjugate $-\Delta_{g}$ by the weight $y_{1}^{-i \lambda+\frac{n}{2}}$.

- the desired smoothness properties should be stronger in the sense that the functions should be smooth in $\left(y_{1}^{2}, y^{\prime}\right)$.

Motivated by this we calculate,

$$
y_{1}^{i \lambda-\frac{n}{2}}\left(-\Delta_{g}-\lambda^{2}-\left(\frac{n}{2}\right)^{2}\right) y_{1}^{-i \lambda+\frac{n}{2}}=x_{1} P(\lambda), \quad x_{1}=y_{1}^{2}, \quad x^{\prime}=y^{\prime},,
$$

where, near $\partial M$,

$$
P(\lambda)=4\left(x_{1} D_{x_{1}}^{2}-(\lambda+i) D_{x_{1}}\right)-\Delta_{h}+i \gamma(x)\left(2 x_{1} D_{x_{1}}-\lambda-i \frac{n-1}{2}\right) .
$$

\footnotetext{
8 A related approach to meromorphic continuation, also motivated by the study of Anti-de Sitter black holes, was independently developed by Warnick [269]. It is based on physical space techniques for hyperbolic equations and it also provides meromorphic continuation of resolvents for even asymptotically hyperbolic metrics [269, §7.5].
} 
We note that (3.11) makes sense globally since away from the boundary $y_{1}$ is a smooth non-zero function on $M$.

To define the operator $P(\lambda)$ geometrically we introduce a new manifold using coordinates (3.3) and $x_{1}=y_{1}^{2}$ for $y_{1}>0$ :

$$
X=[-1,1]_{x_{1}} \times \partial M \sqcup\left(M \backslash y^{-1}((0,1))\right)
$$

We note that $X_{1}:=X \cap\left\{x_{1}>0\right\}$ is diffeomorphic to $M$ but $\bar{X}_{1}$ and $\bar{M}$ have different $\mathcal{C}^{\infty}$-structures. We can extend $x_{1} \rightarrow h\left(x_{1}\right)$ to a family of smooth non-degenerate metrics on $\partial M$ on $[-1,1]$. Using (3.9) that provides a natural extension of the function $\gamma$ appearing (3.11). If in local coordinates we define

$$
d \mu_{g}=\bar{h}(x) d x
$$

then with respect to $L^{2}=L^{2}\left(X, d \mu_{g}\right)$ we have

$$
P(\lambda)^{*}=P(\bar{\lambda})
$$

To define spaces on which $P(\lambda)$ is a Fredholm operator we recall the notation for Sobolev spaces on manifolds with boundary. We denote by $\bar{H}^{s}\left(X^{\circ}\right)$ the space of restrictions of elements of $H^{s}$ on an extension of $X$ across the boundary to the interior of $X$.

Definition 3 We define the following Hilbert spaces

$$
\mathscr{Y}_{s}:=\bar{H}^{s}\left(X^{\circ}\right), \quad \mathscr{X}_{s}:=\left\{u \in \mathscr{Y}_{s+1}: P(\lambda) u \in \mathscr{Y}_{s}\right\}
$$

and the operator $P(\lambda): \mathscr{X}_{s} \rightarrow \mathscr{Y}_{s}$, given by (3.11) and (3.12).

Since the dependence on $\lambda$ in $P(\lambda)$ occurs only in lower order terms we can replace $P(\lambda)$ by $P(0)$ in (3.16). Hence the definition of $\mathscr{X}_{s}$ is independent of $\lambda$.

Motivation: Since for $x_{1}<0$ the operator $P(\lambda)$ is hyperbolic with respect to the surfaces $x_{1}=a<0$ we can motivate Definition 3 as follows. Consider $P=D_{x_{1}}^{2}-D_{x_{2}}^{2}$ on $[-1,0] \times \mathbb{S}^{1}$ and define

$$
\begin{aligned}
Y_{s} & :=\left\{u \in \bar{H}^{s}\left([-1, \infty) \times \mathbb{S}^{1}\right): \operatorname{supp} u \subset[-1,0] \times \mathbb{S}^{1}\right\} \\
X_{s} & :=\left\{u \in Y_{s+1}: P u \in Y_{S}\right\} .
\end{aligned}
$$

Then standard hyperbolic estimates — see for instance [137, Theorem 23.2.4]—show that for any $s \in \mathbb{R}$, the operator $P: X_{s} \rightarrow Y_{s}$ is invertible. Roughly, the support condition gives 0 initial values at $x_{1}=0$ and hence $P u=f$ can be uniquely solved for $x_{1}<0$.

We can now state the result about mapping properties of $P(\lambda)$ : 
Theorem 7 Let $\mathscr{X}_{s}, \mathscr{Y}_{s}$ be defined in (3.16). Then for $\operatorname{Im} \lambda>-s-\frac{1}{2}$ the operator $P(\lambda): \mathscr{X}_{s} \rightarrow \mathscr{Y}_{s}$, has the Fredholm property, that is $\operatorname{dim}\left\{u \in \mathscr{X}_{s}: P(\lambda) u=0\right\}<\infty$, $\operatorname{dim} \mathscr{Y}_{s} / P(\lambda) \mathscr{X}_{s}<\infty$, and $P(\lambda) \mathscr{X}_{s}$ is closed.

Moreover for $\operatorname{Im} \lambda>0, \lambda^{2}+\left(\frac{n}{2}\right)^{2} \notin \operatorname{Spec}\left(-\Delta_{g}\right)$ and $s>-\operatorname{Im} \lambda-\frac{1}{2}, P(\lambda)$ : $\mathscr{X}_{s} \rightarrow \mathscr{Y}_{s}$ is invertible. Hence, for $s \in \mathbb{R}$ and $\operatorname{Im} \lambda>-s-\frac{1}{2}, \lambda \mapsto P(\lambda)^{-1}: \mathscr{Y}_{s} \rightarrow$ $\mathscr{X}_{s}$, is a meromorphic family of operators with poles of finite rank.

In view of (3.11) this immediately recovers the results of Mazzeo-Melrose [176] and Guillarmou [115] in the case of even metrics (Guillarmou showed that for generic non-even metrics global meromorphic continuation does not hold; he also showed that the method of [176] does provide a meromorphic continuation to $\mathbb{C} \backslash-i \mathbb{N}^{*}$ for all asymptotically hyperbolic metrics and to $\mathbb{C}$ for the even ones):

Theorem 8 Suppose that $(M, g)$ is an even asymptotically hyperbolic manifold and that $R(\lambda)$ is defined by (3.7). Then $R(\lambda): \dot{\mathcal{C}}^{\infty}(M) \rightarrow \mathcal{C}^{\infty}(M)$, continues meromorphically from $\operatorname{Im} \lambda>\frac{n}{2}$ to $\mathbb{C}$ with poles of finite rank.

For self-contained proofs of these theorems we refer to [282], and for the semiclassical version in the same spirit to $[80, \S 5.6]$. The general idea is to estimate $u$ in terms of $P(\lambda) u$ with lower order corrections:

$$
\|u\|_{\bar{H}^{s+1}\left(X^{\circ}\right)} \leq C\|P(\lambda) u\|_{\bar{H}^{s}\left(X^{\circ}\right)}+C\|\chi u\|_{H^{-N}(X)},
$$

where $\chi \in \mathcal{C}_{\mathrm{c}}^{\infty}\left(X^{\circ}\right), \chi=0$ in $x_{1}<-2 \delta, \chi=1$ in $x_{1}>-\delta, \delta>0$. That is done by using ellipticity of $P(\lambda)$ in $x_{1}>\delta$, hyperbolicity of $P(\lambda)$ in $x_{1}<-\delta$ and propagation of singularities in the transition region $x_{1}=0$ - see [282, §4,5].

The key propagation estimate used by Vasy [263] comes from the work of Melrose on propagation estimates at radial points occurring in scattering on asymptotically Euclidean spaces [183]. These estimates also play a role in applications of microlocal methods to dynamical systems-see Sect. 4. Here we will only state one basic consequence—see [282, §4, Remark 3]:

$$
P(\lambda) u \in \overline{\mathcal{C}}^{\infty}(X), \quad u \in \bar{H}^{s+1}(X), \quad s>-\operatorname{Im} \lambda-\frac{1}{2} \Longrightarrow u \in \overline{\mathcal{C}}^{\infty}(X) .
$$

This means that above a threshold of regularity given by $-\operatorname{Im} \lambda+\frac{1}{2}$ the operator is hypoelliptic and that

$$
\operatorname{ker}_{\mathscr{X}_{s}} P(\lambda) \neq\{0\}, \quad s>-\operatorname{Im} \lambda-\frac{1}{2} \Longleftrightarrow \exists u \in \overline{\mathcal{C}}^{\infty}(X), P(\lambda) u=0
$$

This implies that resonant states, in the original coordinates, are characterized by

$$
\left(-\Delta_{g}-\lambda^{2}-\left(\frac{n}{2}\right)^{2}\right) w=0, \quad y_{1}^{i \lambda-\frac{n}{2}} w \in \overline{\mathcal{C}}^{\infty}(M)
$$

which should be compared to (2.24). 
Some understanding of (3.17) can be obtained as follows. Suppose we consider a simplified operator $P_{0}(\lambda)=x D_{x}^{2}-(\lambda+i) D_{x}$, Then solutions of $P_{0}(\lambda) u=0$ are given by

$$
u(x)=a_{+} x_{+}^{i \lambda}+a_{-} x_{-}^{i \lambda}+b, \quad \lambda \notin-i \mathbb{N}, \quad x \in \mathbb{R} .
$$

Here we use the notation of [136, §3.2]. From [136, Example 7.1.17] we then see that $x_{ \pm}^{i \lambda} \in H_{\mathrm{loc}}^{-\operatorname{Im} \lambda+\frac{1}{2}-} \backslash H_{\mathrm{loc}}^{-\operatorname{Im} \lambda+\frac{1}{2}}$. Hence,

$$
u \in \bar{H}^{s+1}((-1,1)), s>-\operatorname{Im} \lambda-\frac{1}{2} \Longrightarrow a_{ \pm}=0 \Longrightarrow u \in \overline{\mathcal{C}}^{\infty}((-1,1)) .
$$

This also shows that (3.17), and consequently Theorem 7 , are essentially optimal.

The actual proof of (3.17) is based on the analysis of the Hamilton flow of the principal symbol of $P(\lambda), x_{1} \xi_{1}^{2}+\left|\xi^{\prime}\right|_{h(x)}^{2} \in \mathcal{C}^{\infty}\left(T^{*} X\right)$, and of positive commutator estimates depending on lower order terms - that is where the dependence on $\lambda$ comes from. For that we refer to [282, §4], [80, §E.5.2].

One weakness of the method lies in the fact that it provides effective meromorphic continuation only in strips, even though the resolvent is meromorphic in $\mathbb{C}$. Hence, results which involve larger regions (such as asymptotics of resonances for convex obstacles [152,247] where resonances lie in cubic regions, $\left.\operatorname{Im} \lambda \sim-|\operatorname{Re} \lambda|^{\frac{1}{3}}\right)$ are still inaccessible in the setting of asymptotically hyperbolic manifolds (or even $\mathbb{H}^{n} \backslash \mathcal{O}$ ). Analyticity near infinity should play a role when larger regions are considered and towards that aim we formulate a conjecture which could perhaps interest specialists in analytic hypoellipticity. It is an analytic analogue of (3.17) and it also has a microlocal version:

Conjecture $2{ }^{9}$ Suppose that $P(\lambda)$ is given by (3.12) and that near $x_{1}=0$ the coefficients of $P(\lambda)$ are real analytic. Let $U$ be a sufficiently small neighbourhood of $x_{1}=0$. Then,

$$
P(\lambda) u \in C^{\omega}(U), \quad u \in H^{s+1}(U), s>-\operatorname{Im} \lambda-\frac{1}{2} \Longrightarrow u \in C^{\omega}(U),
$$

where $C^{\omega}$ denotes the space of analytic functions.

We remark that in the analytic case the operator $P(\lambda)$ belongs to the class of Fuchsian differential operators studied by Baouendi-Goulaouic [11] and that the conjecture is true for $P(\lambda)=4\left(x_{1} D_{x_{1}}^{2}-(\lambda+i) D_{x_{1}}\right)-\Delta_{h}$, where $h$ is a metric on $\partial M$, independent of $x_{1}$ and $\lambda \notin-i \mathbb{N}^{*}[166]$.

\subsection{Resonance free regions}

We will now consider semiclassical operators

$$
P=P(h):=-h^{2} \Delta_{g}+V
$$

\footnotetext{
9 After this survey first appeared Conjecture 2 was proved by Claude Zuily [275] who used results of Bolley-Camus [24] and Bolley-Camus-Hanouzet [25]. These methods also showed analyticity of radiation patterns of resonant states, $F$ in (3.10), also when the metric is not even. The award of the prize (see footnote 6) took place at http://www.latabledulancaster.fr on November 30, 2016.
} 


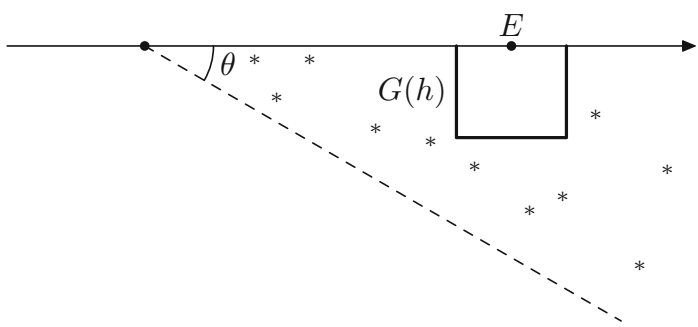

Fig. 20 The meromorphic continuation (3.21): The resonances of $P$ are identified with the eigenvalues the scaled operator $P_{\theta}$ constructed by a higher dimensional version of the method presented in Sect. 2.6. Resonances are studied near a fixed energy level in a neighbourhood of size depending on $h$

on Riemannian manifolds $(M, g)$ where $M$ is isometric to $\left(\mathbb{R}^{n}, g_{0}\right)$ outside of a compact set, with $g_{0}$ the Euclidean metric, and $V \in \mathcal{C}_{\mathrm{c}}^{\infty}\left(\mathbb{R}^{n} ; \mathbb{R}\right)$. More general classes of metrics and potentials can be considered-see [241]. We could also generalize the class of manifolds-see [273]. Later in this section we will also discuss the case of asymptotically hyperbolic manifolds of Sect. 3.1.

The method of complex scaling (see [241,273]) gives (see Fig. 20)

$$
\begin{aligned}
(P-z)^{-1}: \mathcal{C}_{\mathrm{c}}^{\infty}(M) \rightarrow \mathcal{C}^{\infty}(M) & \text { continues meromorphically from } \operatorname{Im} z>0 \\
\text { to } \operatorname{Im} z & >-\theta \operatorname{Re} z, \operatorname{Re} z>0 .
\end{aligned}
$$

We denote the set of poles of $(P(h)-z)^{-1}$, that is the set of resonances of $P(h)$, by $\operatorname{Res}(P(h))$ (we include $h$ to stress the dependence on our semiclassical parameter).

The asymptotic parameter $h$ is supposed to be small and we will consider resonances of $P(h)$ near a fixed energy level. When $V \equiv 0$ then the limit $h \rightarrow 0$ corresponds to the high energy limit but even in that case the link with physical intuitions around classical/quantum correspondence is useful.

The distribution of resonances is closely related to the properties of the classical Hamiltonian flow

$$
\varphi_{t}: T^{*} M \rightarrow T^{*} M, \varphi_{t}:=\exp \left(t H_{p}\right), \quad p:=|\xi|_{g}^{2}+V(x),
$$

where $T^{*} M$ is the cotangent bundle of $M$, and $H_{p}$ is the Hamilton vector field of $p$. In local coordinates $(x, \xi) \in T^{*} M, x \in M$,

$$
H_{p}=\sum_{j=1}^{n} \partial_{\xi_{j}} p \partial_{x_{j}}-\partial_{x_{j}} p \partial \xi_{j} .
$$

To define the trapped set we first define incoming and outgoing sets at a given interval of energies $J \subset \mathbb{R}$ :

$$
\begin{gathered}
\Gamma_{J}^{ \pm}:=\left\{(x, \xi) \in p^{-1}(J): \pi\left(\varphi_{t}(x, \xi)\right) \not \rightarrow \infty \text { as } t \rightarrow \mp \infty\right\}, \\
\pi: T^{*} M \rightarrow M, \pi(x, \xi):=x .
\end{gathered}
$$

and the trapped set at energy $E$ : 


$$
K_{J}=\Gamma_{J}^{+} \cap \Gamma_{J}^{-}
$$

If $J=\{E\}$ then we write $K_{\{E\}}=K_{E}$. The simplest is "non-trapping scattering" in which $K_{E}=\emptyset$. This implies that $K_{J}=\emptyset$ for some neighbourhood $J$ of $E$-see [80, $\S 6.1]$ for other basic properties of $\Gamma^{ \pm}$and $K$.

The behaviour of the flow near the trapped set has an effect on the distribution of scattering resonances and in particular on the type of resonance free regions. As in Sect. 2.4 we consider different possibilities:

$$
\operatorname{Im} z>-G(h), \quad|\operatorname{Re} z-E|<\delta, \quad G(h)=\left\{\begin{array}{l}
\left(\text { a) } e^{-\alpha / h}, \alpha>0\right. \\
(b) \gamma h \\
(c) M h \log \frac{1}{h} \\
\text { (d) } \gamma h^{\beta}, \beta \in \mathbb{R}, \gamma>0
\end{array}\right.
$$

In the case of $P(h)=-h^{2} \Delta_{g}$ (or for obstacle problems) this is equivalent to resonance free regions (2.45) with

$$
F(x)=2 x G(1 / x) .
$$

Note that $\lambda^{2}=h^{2} z$; this is consistent with dynamical quantities defined for $p=|\xi|_{g}^{2}$ in the $z$-picture and for $p=|\xi|_{g}$ in the $\lambda$ picture. Nevertheless this is potentially confusing when comparing results involving (3.29) and (3.36).

\begin{tabular}{|c|c|}
\hline Hamiltonian flow & Resonance free region and resolvent bounds \\
\hline General case & $\begin{array}{l}\text { (a) }[38,43,51,224,236,268],[80, \S 6.4] \text {; optimal for shape } \\
\text { resonances }[95,125] \text {, and for "resonances from quasimodes" } \\
{[102,254,260],[80, \S 7.3] \text {; corresponding cut-off resolvent }} \\
\text { bounds (cf.(2.46)) also optimal [54] }\end{array}$ \\
\hline Normally hyperbolic trapping & $\begin{array}{l}\text { (b) with } \gamma \text { given by a "Lyapounov exponent" }[69,108,202,274] \text {, } \\
\text { [80, } \$ 6.3] \text {; optimal for one closed hyperbolic orbit }[107] \text { and for } \\
r \text {-normally hyperbolic trapping [67] }\end{array}$ \\
\hline Hyperbolic trapped set & $\begin{array}{l}\text { (b) with } \gamma \text { determined by a topological pressure of the trapped set } \\
{[200] \text {; an improved gap expected; known for hyperbolic quotients }} \\
{[75,191,257]}\end{array}$ \\
\hline Smooth non-trapping & (c) with arbitrarily large $M[174,248]$ \\
\hline$a$-Gevrey non-trapping & (d) with $\beta=1-1 / a$ [225] \\
\hline Analytic non-trapping & $\begin{array}{l}\text { (d) with } \beta=0[125,240] \text {; optimal with density } \sim h^{-n} \text { via } \\
\text { Sjöstrand's trace formula [242], [80, §7.4] }\end{array}$ \\
\hline
\end{tabular}

The following table indicates various stable dynamical configurations with pointers to the literature (we refer to (3.26) for the types of resonance free regions):

(See Definitions 4, 5 for definitions hyperbolic and normally hyperbolic trapped sets and (3.29), (3.36) for definitions of "Lyapounov exponenents" and topogical pressure respectively; smooth, Gevrey and analytic refers to the regularity of the coefficients of the operator $P$.)

The first case we will consider is that of hyperbolic trapped sets: 
Definition 4 Suppose that $\left.d p\right|_{p^{-1}(E)} \neq 0$. We say that the flow $\varphi^{t}$ is hyperbolic on $K_{E}$, if for any $\rho \in K_{E}$, the tangent space to $p^{-1}(E)$ at $\rho$ splits into flow, unstable and stable subspaces:

$$
\begin{aligned}
& \text { i) } T_{\rho}\left(p^{-1}(E)\right)=\mathbb{R} H_{p}(\rho) \oplus E_{\rho}^{+} \oplus E_{\rho}^{-}, \quad \operatorname{dim} E_{\rho}^{ \pm}=n-1 \\
& \text { ii) } d \varphi_{\rho}^{t}\left(E_{\rho}^{ \pm}\right)=E_{\varphi^{t}(\rho)}^{ \pm}, \quad \forall t \in \mathbb{R} \\
& \text { iii) } \exists \lambda>0 \text {, and a smooth metric } \rho \mapsto\|\bullet\|_{\rho} \text { such that } \\
& \left\|d \varphi_{\rho}^{t}(v)\right\|_{\varphi^{t}(\rho)} \leq e^{-\lambda|t|}\|v\|_{\rho}, \text { for all } v \in E_{\rho}^{\mp}, \pm t \geq 0 .
\end{aligned}
$$

For more on this in dynamical systems see $[154, \S 17.4]$. One important property is the stability of this condition. We also remark that the existence of the metric $\|\bullet\|_{\rho}$ in part (iii) or (3.28) follows from the same estimates for some metric with $C e^{-\lambda|t|}$ on the right hand side. Examples include $p=|\xi|_{g}^{2}$ where the curvature is negative near the trapped set or $p=|\xi|^{2}+V(x)$ where $V$ is a potential given by several bumps (see [202, Figure 1]). In obstacle scattering trapped sets are hyperbolic for several convex obstacles satisfying Ikawa's non-eclipse condition (see [198, (1.1)]).

A dynamically defined function which plays a crucial role here is the topological pressure. We present a definition valid when closed trajectories are dense in $K_{E}$ - that is the case in the examples mentioned above. Let $\gamma$ denote a closed trajectory of $\varphi_{t}$ on $p^{-1}(E)$ and $T_{\gamma}$ its length. We then define

$$
\begin{aligned}
\mathcal{P}_{E}(s) & :=\lim _{T \rightarrow \infty} \frac{1}{T} \log \sum_{T \leq T_{\gamma} \leq T+1} J^{+}(\gamma)^{-s}, \\
J^{+}(\gamma) & :=\int_{0}^{T_{\gamma}} \varphi^{+}\left(\varphi_{t}\left(\rho_{\gamma}\right)\right) d t, \quad \rho_{\gamma} \in \gamma, \quad \varphi^{+}(\rho):=\left.\frac{d}{d t} \log \operatorname{det}\left(\left.d \varphi_{t}(\rho)\right|_{E_{\rho_{\gamma}}} ^{+}\right)\right|_{t=0}
\end{aligned}
$$

and the expression is independent of the choice of $\rho_{\gamma} \in \gamma$ and of the density defining the determinant. (Strictly speaking this is the pressure associated to $-s \varphi^{+}$but as that is the only one we will consider we just call it the pressure.)

This definition is not the one that is in fact useful in the study of resonances but it is the simplest one to state. It can be interpreted as follows: because of (iii) in (3.28) we have $J^{+}(\gamma)>1$ (here we choose the determinant using the density coming from the metric $\|\bullet\|_{\rho}$ ) and this expression measures the averaged rate of expansion in ustable directions $E_{\rho_{\gamma}}^{+}$along the closed orbit $\gamma$. Hence $J^{+}(\gamma)^{-s}$ gets smaller with increasing $s$. On the other hand, the count over closed orbits of length $T_{\gamma} \sim T$ measures the complexity of the dynamical system: it is more complex if there are more closed orbits of lengths in $[T, T+1]$. It follows that for $s>0, \mathcal{P}_{E}(s)$ measures the fight between complexity and dispersion in the unstable directions. If $\mathcal{P}_{E}\left(s_{0}\right)=0$ then for $s>s_{0}$ the dispersion wins. For two dimensional systems the Bowen pressure formula shows that the Hausdorff dimension of the trapped set is given in terms of $s_{0}$ :

$$
\operatorname{dim} K_{E}=2 s_{0}+1
$$



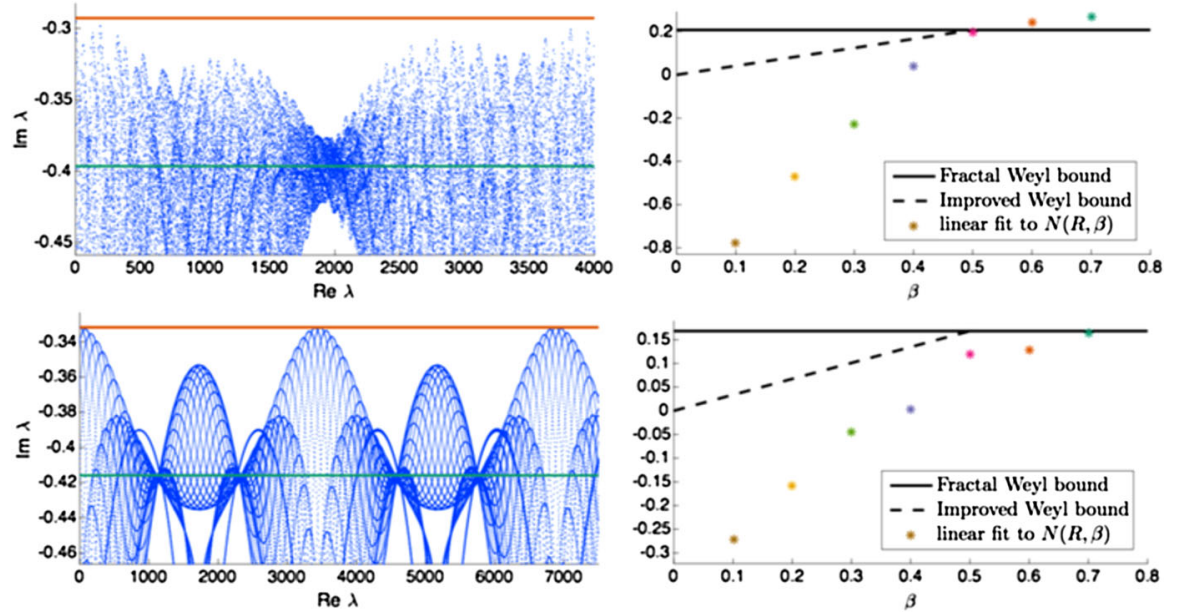

Fig. 21 Left resonances for $X(6,7,7)$ and $Y(7,7, \pi / 2)$ from [70, Appendix] (see Fig. 19 for the definition and picture of these surfaces; here we show the poles of the continuation of $\left.\left(-\Delta_{g}-\lambda^{2}-\frac{1}{4}\right)^{-1}\right)$. The top line is the pressure gap (3.33) with $P\left(\frac{1}{2}\right)=\frac{1}{2}-\delta$. The lower line is given by half of the classical decay rate, $P(1) / 2=(1-\delta) / 2$ where $\delta$ is the dimension of the limit sets for the two surfaces-see (3.31). Right numerical illustration of Theorems 13 and 14 with the standard [53,122] and improved [70] fractal Weyl laws-see Sect. 3.4-for the corresponding surfaces; here $N(R, \beta)$ is the number of resonances in $|\operatorname{Re} \lambda| \leq R, \operatorname{Im} \lambda>\frac{1}{2}+(\beta-1) \delta$. The "chain patterns" visible on the left (which are even more pronounced for more symmetric systems such as $X(\ell, \ell, \ell)$ — see [33]) have been recently investigated by Weich [270]

— see for instance [143] and references given there. When $p=|\xi|_{g}^{2}$ on a convex cocompact surface, $M=\Gamma \backslash \mathbb{H}^{2}$ - see Fig. 19-then, in that special constant curvature case, we have for any $E>0$,

$$
\begin{aligned}
\operatorname{dim} K_{E} & =2 \delta+1, \mathcal{P}_{E}(s)=2 E^{-\frac{1}{2}}(\delta-s), \quad \delta:=\operatorname{dim} \Lambda(\Gamma), \\
\Lambda(\Gamma) & :=\overline{\Gamma z} \cap \partial \mathbb{H}^{2} .
\end{aligned}
$$

The set $\Lambda(\Gamma)$ is called the limit set of $\Gamma$ and the definition does not depend on the choice of $z \in \mathbb{H}^{2}$ - see [31] and references given there.

The analogue of Ikawa's result for several convex obstacles [140], postulated independently in the physics literature by Gaspard-Rice [103], was established for operators of the form (3.20) by Nonnenmacher-Zworski [200,201]. Thanks to the results of Sect. 3.1 the result also holds for $-h^{2} \Delta_{g}$ on even asymptotically hyperbolic manifolds. In that case it generalizes celebrated results of Patterson and Sullivan (see [31]) formulated using dimension of the limit set (3.31). That gap is shown as the top line on the right graphs in Fig. 21.

Theorem 9 Suppose that an operator (3.20) has a hyperbolic trapped set at energy $E$, in the sense of Definition 4. If the pressure defined in (3.29) satisfies

$$
0>\mathcal{P}_{E}\left(\frac{1}{2}\right)
$$

then there are no resonances near $E$ with $\operatorname{Im} z>h \mathcal{P}_{E}\left(\frac{1}{2}\right)$. 
More precisely, if $\gamma_{E}(\delta):=\min _{\left|E-E^{\prime}\right| \leq \delta}\left(-\mathcal{P}_{E^{\prime}}\left(\frac{1}{2}\right)\right)$ then

$$
\exists \delta>0 \forall \gamma<\gamma_{E}(\delta) \exists h_{0} \operatorname{Res}(P(h)) \cap([E-\delta, E+\delta]-i h[0, \gamma])=\emptyset,
$$

when $h<h_{0}$. In addition for any $\chi \in \mathcal{C}_{\mathrm{c}}^{\infty}(M)$, we have, for some $c, C>0$,

$$
\left\|\chi(P-z)^{-1} \chi\right\|_{L^{2} \rightarrow L^{2}} \leq C h^{-1+c \operatorname{Im} z / h} \log \frac{1}{h},
$$

for $z \in[E-\delta, E+\delta]-i h[0, \gamma]$.

It is not clear if the pressure condition (3.32) is needed to obtain a resonance stripsee Theorem 11 below. What seems to be clear is that $\mathcal{P}_{E}\left(\frac{1}{2}\right)$ is a robust classical quantity determining the strip while the improvements require analysing quantum effects. The upper bound (3.34) is optimal for $\operatorname{Im} z=0$ thanks to a general result of Bony-Burq-Ramond [27], [80, §7.1].

Applications of the estimate (3.34) include local smoothing estimates with a logarithmic loss by Datchev [50] and Strichartz estimates with no loss by BurqGuillarmou-Hassell [39]. The method of proof was used by Schenck [232] to obtain decay estimates for damped wave equations and by Ingremeau [141] to describe distorted plane waves in quantum chaotic scattering.

For an outline of the proof and a more relevant description of the pressure function we refer to [200, §2] and to an excellent survey by Nonnenmacher [196, §8.1] where a discussion of optimality can also be found. Here we make one general comment relevant also in the case of Theorem 10 below. Thanks to the "gluing" results of DatchevVasy [57] one can prove existence of the pole free region (3.33) and the cut-off resolvent bound (3.34) for a simpler operator modified by a complex absorbing potential: ${ }^{10}$

$$
P_{W}:=-h^{2} \Delta_{g}+V(x)-i W, \quad W \in \mathcal{C}^{\infty}(M ;[0,1]),
$$

with $W(x) \equiv 1$ for $|x|>R$ where $R$ is chosen large enough. The operators $P_{W}-z: H^{2} \rightarrow L^{2}$ are Fredholm operators for $\operatorname{Im} z>-1$ and are invertible for $\operatorname{Im} z>0$. As shown in $[202, \S 8]$ the estimates on $\left(P_{W}-z\right)^{-1}$ imply estimates (3.34) while being easier to obtain.

The trapped sets to which Theorem 9 applies are typically fractal. In fact, in dimension 2 the condition $\mathcal{P}_{E}\left(\frac{1}{2}\right)<0$ is equivalent to the trapped set being filamentary in the sense that $\operatorname{dim} K_{E}<2$, that is the trapped set is below the mean of the maximal dimension 3 and the minimal dimension 1 (direction of the flow). We will now consider another case in which the trapped set is smooth but with hyperbolicity in the transversal direction:

Definition 5 We say that the Hamiltonian flow is normally hyperbolic at energy $E$ if for some $\delta$ and $J=[E-\delta, E+\delta], K_{J}$ is a smooth symplectic submanifold of $T^{*} M$,

\footnotetext{
10 This method is related to a method used in chemistry to compute resonances — see [223] and [235] for the original approach and [145] for some recent developments and references. A simple mathematical result justifying this computational method is given in [283]: if $V \in L_{\text {comp }}^{\infty}\left(\mathbb{R}^{n}\right)$ then the eigenvalues of $-\Delta+V-i \varepsilon x^{2}$ converge to resonances of $-\Delta+V$ uniformly on compact subsets of the region $\arg z>-\pi / 4$.
} 
and there exists a continuous distribution of linear subspaces, $K_{J} \ni \rho \longmapsto E_{\rho}^{ \pm} \subset$ $T_{\rho}\left(T^{*} M\right)$, invariant under the flow, $d \varphi_{t}\left(E_{\rho}^{ \pm}\right)=E_{\varphi_{t}(\rho)}^{ \pm}$, satisfying, $\operatorname{dim} E_{\rho}^{ \pm}=d_{\perp}$ and

$$
\begin{aligned}
T_{\rho} K_{J} \cap E_{\rho}^{ \pm}= & E_{\rho}^{+} \cap E_{\rho}^{-}=\{0\}, \quad T_{\rho}\left(T^{*} X\right)=T_{\rho} K^{\delta} \oplus E_{\rho}^{+} \oplus E_{\rho}^{-}, \\
& \forall v \in E_{\rho}^{ \pm}, \quad \forall t>0, \quad\left\|d \varphi_{\mp t}(\rho) v\right\|_{\varphi_{\mp t}(\rho)} \leq C e^{-\lambda t}\|v\|_{\rho},
\end{aligned}
$$

form some smoothly varying norm on $T_{\rho}\left(T^{*} M\right), \rho \mapsto\|\bullet\|_{\rho}$.

This dynamical configuration is stable under perturbations under a stronger addition condition of $r$-normal hyperbolicity. Roughly speaking that means that the flow on $K_{J}$ has weaker expansion and contraction rates than the flow in the transversal directions - see [67,132] for precise a definition and (3.48) below for an example. Normally hyperbolic trapping occurs in many situations: for instance, for null flows for black hole metrics, see [76], Sect. 3.3, and in molecular dynamics, see [202, Remark 1.1], [111,234]. Another important example comes from contact Anosov flows lifted to the cotangent bundle, see [261], [202, Theorem 4], Sect. 4.4. The following general result was proved by Nonnenmacher-Zworski [202]:

Theorem 10 Suppose that for $P$ given by (3.20) and that at energy $E$ the trapped set is hyperbolic in the sense of Definition 5. Define the minimal expansion rate by

$$
v_{\min }:=\liminf _{t \rightarrow \infty} \frac{1}{t} \inf _{\rho \in K_{E}} \log \operatorname{det}\left(\left.d \varphi_{t}\right|_{E_{\rho}^{+}}\right) .
$$

Then near $E$ there are no resonances with $\operatorname{Im} z>-h v_{\min } / 2$. More precisely

$$
\exists \delta \forall \varepsilon>0 \exists h_{0} \operatorname{Res}(P(h)) \cap\left([E-\delta, E+\delta]-i h \frac{1}{2}\left[0, \mu_{\min }-\varepsilon\right]\right)=\emptyset,
$$

and the estimate (3.34) holds in this resonance free region.

For more general trapped sets but for analytic coefficients of $P$ and without the estimate (3.34), the resonance free region (3.37) was obtained early on by GérardSjöstrand [108]. Sometimes, for instance in the case of black holes, $d^{\perp}=1$ (see 3.35), $\Gamma_{J}^{ \pm}$(see 3.24) are smooth, and $T_{\rho} K_{J} \oplus E_{\rho}^{+}=T_{\rho} \Gamma_{J}^{ \pm}$. In that case a resonance free region $\operatorname{Im} z>-h / C$ and the bound (3.34) was obtained by Wunsch-Zworski [274]. An elegant proof with the width (3.36) and a sharp resolvent estimate was given by Dyatlov [69][80, §6.3]. The width given by $v_{\min }$ is essentially optimal as one can already see from the work of Gérard-Sjöstrand [107] on the pseudo-lattice of resonances generated by a system with one hyperbolic closed orbit. A general result was provided by Dyatlov [67]: if $K_{E}$ is $r$-normally hyperbolic for any $r$ and if the maximal expansion rate $v_{\max }$ satisfies $v_{\max }<2 v_{\min }$, then the resonance free region is essentially optimal in the sense that in the strip below $\operatorname{Im} z=h v_{\min } / 2$ there exist infinitely many resonances-see Fig. 22.

We now discuss improvements over the pressure gap. The first such improvement for scattering resonances was achieved by Naud [191] who extended Dolgopyat's method [59] to the case of convex co-compact quotients and showed that there exists $\gamma>0$ such that when $\delta<\frac{1}{2}$ (see 3.31) then there are no resonances other than $i\left(\delta-\frac{1}{2}\right)$ with $\operatorname{Im} \lambda>\delta-\frac{1}{2}-\gamma$. The Dolgopyat method was further developed by Stoyanov 


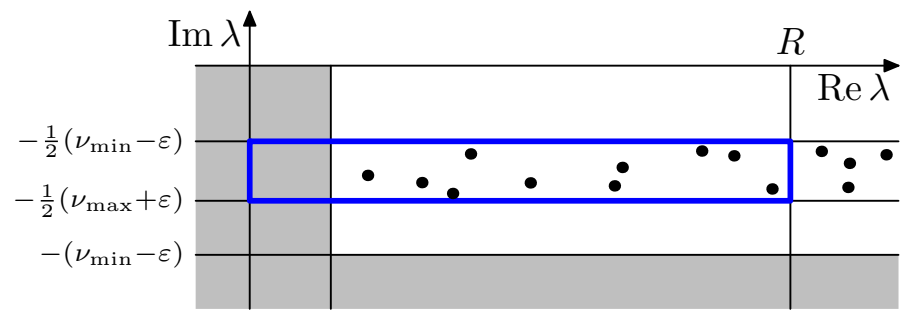

Fig. 22 A schematic presentation of the results of [67] for an operator $-\Delta_{g}$ with an $r$-normally hyperbolic trapped set. Here $R=1 / h$ and we use the resolvent $\left(-\Delta_{g}-\lambda^{2}\right)^{-1}$. The resonance free strip is the same as in Theorem 10 and the resonances in the strip $\operatorname{Im} \lambda>-\frac{1}{2}\left(v_{\max }+\varepsilon\right)$ satisfy a Weyl law: $\mid\{\lambda: \operatorname{Im} \lambda>$ $\left.-\frac{1}{2}\left(v_{\max }+\varepsilon\right), 0<\operatorname{Re} \lambda<R\right\} \mid \sim R^{n-1} \operatorname{vol}\left(K_{[0,1]}\right) /(2 \pi)^{n-1}$ —see [67, Theorem 3]

[257] for higher dimensional quotients (as an application of general results) and by Oh-Winter [203] for uniform gaps for arithmetic quotients. Petkov-Stoyanov [210] also adapted Dolgopyat's method to the case of several convex obstacles. All of these results assume that $\mathcal{P}_{E}\left(\frac{1}{2}\right) \leq 0$.

A new method for obtaining improved resonance free regions, applicable also when $\mathcal{P}_{E}\left(\frac{1}{2}\right)>0$, was introduced by Dyatlov-Zahl [75]. It is based on a fractal uncertainty principle (FUP) which in [75] was combined with an investigation of additive structure of limit sets (see 3.31) to obtain an improved gap for quotients $\Gamma \backslash \mathbb{H}^{2}$, with $\delta(\Gamma) \approx \frac{1}{2}$. In particular that produced the first resonance free strips when $\mathcal{P}_{E}\left(\frac{1}{2}\right)>0$.

Roughly speaking the standard uncertainty principle says that wave functions cannot be strongly localized in both position and frequency near a point. The fractal uncertainty principle (FUP) states that a wave function cannot be strongly localized in both position and frequency near a fractal set. To describe FUP rigorously we will use a simpler model studied recently by Dyatlov and Jin [74]: open quantum maps with products of exact Cantor sets as trapped sets.

Open quantum maps have been studied in physics and mathematics. They are quantizations of symplectic relations on $\mathbb{T}^{2}=\mathbb{R}^{2} / \mathbb{Z}^{2}$ where the symplectic relations have features of Poincaré maps in scattering theory: see Nonnenmacher [196, $\S 5]$ for a general introduction, [74, §1.4] for references to the physics literature, Nonnenmacher-Sjöstrand-Zworski [197] for a reduction of chaotic scattering problems to quantizations of Poincaré maps and Nonnenmacher-Zworski [199] for quantization of piecewise smooth relations.

An example of a piecewise smooth symplectic relation is shown on the left in Fig. 23: the torus is divided into five "vertical strips", three of them are thrown out and the remaining two are stretched/contracted and then shifted (the caption to Fig. 23 has a general formula for $\kappa_{M, \mathcal{A}}$.) This relation, $\kappa_{5,\{1,3\}}$, is quantized by the following family of matrices:

$$
B_{5 N}:=\mathcal{F}_{5 N}^{*}\left(\begin{array}{ccccc}
0 & 0 & 0 & 0 & 0 \\
0 & \chi_{N} \mathcal{F}_{N} \chi_{N} & 0 & 0 & 0 \\
0 & 0 & 0 & 0 & 0 \\
0 & 0 & 0 & \chi_{N} \mathcal{F}_{N} \chi_{N} & 0 \\
0 & 0 & 0 & 0 & 0
\end{array}\right)
$$




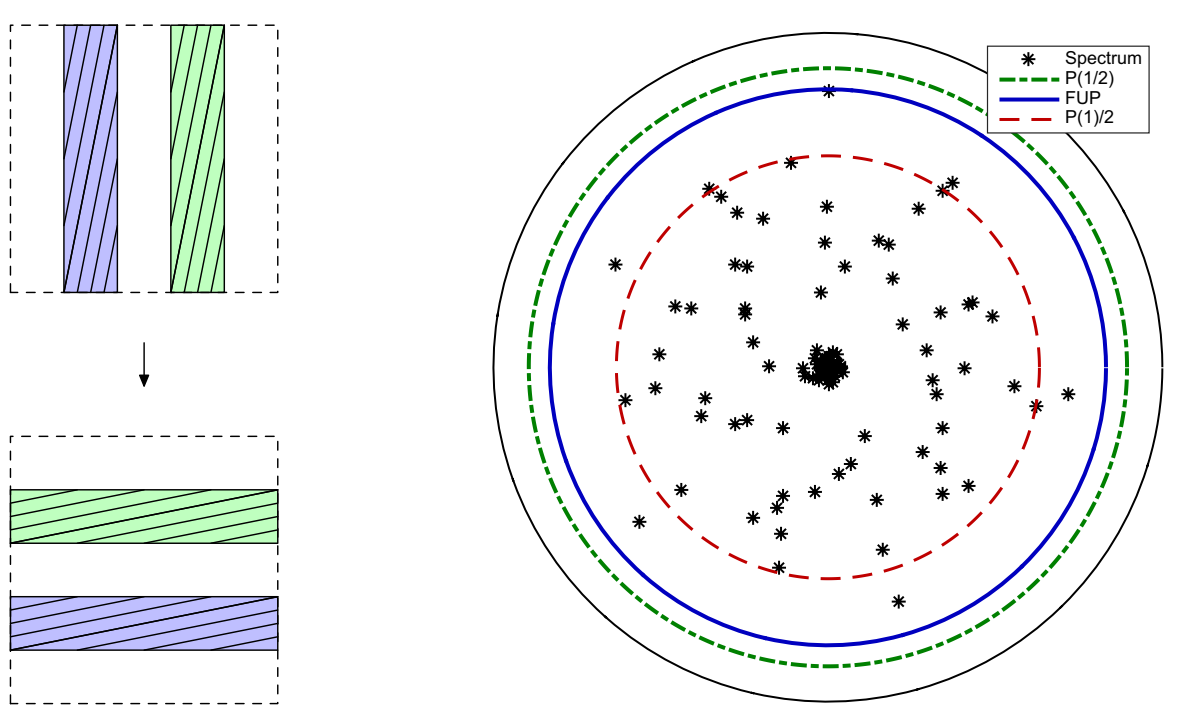

Fig. 23 Left a schematic representation of an open baker map (a piecewise smooth symplectic relation) $\kappa_{M, \mathcal{A}}: \mathbb{R}^{2} / \mathbb{Z}^{2} \ni(y, \eta) \mapsto(M y-a,(\eta+a) / M),(y, \eta) \in(a / M,(a+1) / M), a \in \mathcal{A} \subset\{0, \ldots, M-1\}$, here with $M=5, \mathcal{A}=\{1,3\}$. A quantization, $B_{5 N}$, of this open map (relation) is given in (3.38). Right resonances of $B_{5 N}$ with $N=5^{4}$. Different theoretical gaps are also shown: for this quantum map the fractal uncertainty principle (FUP) of $[74,75]$ does produce an optimal gap

where $\mathcal{F}_{L}$ is the unitary Fourier transform on $\ell_{L}^{2}:=\ell^{2}(\mathbb{Z} / L \mathbb{Z}), \chi_{N}:=\operatorname{diag}(\chi(j / N))$ for $\chi \in \mathcal{C}_{\mathrm{c}}^{\infty}((0,1) ;[0,1])$.

On the classical level a relation $\kappa$ defines discrete time analogues of (3.24) and (3.25):

$$
\Gamma^{ \pm}:=\bigcap_{ \pm r \geq 0} \kappa^{r}\left(\mathbb{T}^{2}\right), \quad K=\Gamma^{+} \cap \Gamma^{-}
$$

In the case of $\kappa_{M, \mathcal{A}}$ we have

$$
\begin{aligned}
\Gamma_{-} & =\mathcal{C} \times \mathbb{S}^{1}, \quad \Gamma_{+}=\mathbb{S}^{1} \times \mathcal{C}, \quad K=\mathcal{C} \times \mathcal{C}, \operatorname{dim} K=2 \delta, \\
\mathcal{C} & :=\bigcap_{k} \bigcup_{j \in \mathcal{C}_{k}}\left[\frac{j}{M^{k}}, \frac{j+1}{M^{k}}\right], \quad \mathcal{C}_{k}:=\left\{\sum_{j=0}^{k-1} a_{j} M^{j}: a_{j} \in \mathcal{A}\right\}, \\
\delta & :=\operatorname{dim} \mathcal{C}=\frac{\log |\mathcal{A}|}{\log M} .
\end{aligned}
$$

If we think of $\mathbb{T}^{2}$ as being an analogue of a hypersurface $\Sigma$ transversal to the flow then $K$ is the analogue $K_{E} \cap \Sigma$. The linearization of $\kappa_{M, \mathcal{A}}$ (at regular points) has eigenvalues $M, M^{-1}$ and hence we can think of $\kappa$ as the flow at time $t=\log M$ with the expansion rate $\lambda=1$ [see (iii) in (3.28)]. The pressure of the suspension at time $\log M$ is given by 


$$
\mathcal{P}(s)=\delta-s,
$$

see [196, §8] for a discussion of pressure for suspensions.

The operator $B_{N}$ is considered as quantization of the propagator at time $t=\log M$ with $h=2 \pi / N$. Hence the conceptual correspondence between eigenvalues of $B_{N}$ and resonances of semiclassical operators $P(h)$ (established in some cases in [197]) is

$$
\operatorname{Spec}\left(B_{N}\right) \ni \lambda=e^{-i t z / h} \longleftrightarrow z \in \operatorname{Res}(P(h)) .
$$

Since $t=\log M$ the correspondence between resonances free regions is given by the following

$$
([1,2]-i h[0, \gamma]) \cap \operatorname{Res}(P(h))=\emptyset \longleftrightarrow \operatorname{Spec}\left(B_{N}\right) \cap\left\{|\lambda| \geq M^{-\gamma}\right\}=\emptyset .
$$

In view of this and (3.40) the analogue of (3.33) is

$$
\forall \varepsilon>0 \quad \operatorname{Spec}\left(B_{N}\right) \cap\left\{|\lambda| \geq M^{\delta-\frac{1}{2}+\varepsilon}\right\}=\emptyset, \quad N \geq N_{\varepsilon},
$$

see $[196, \S 8]$.

The general principle of [75] and [74] for quantization of $\kappa_{M, \mathcal{A}}$ goes as follows. In the notation of (3.38) and (3.39) we say that

$$
\text { FUP holds with exponent } \gamma \Longleftrightarrow\left\|\mathbb{1}_{\mathcal{C}_{k}} \mathcal{F}_{N} \mathbb{1}_{\mathcal{C}_{k}}\right\|_{\ell_{N}^{2} \rightarrow \ell_{N}^{2}} \leq C N^{-\gamma} \text {. }
$$

Then we have (see [74, Proposition 2.7] for this case and [75, Theorem 3] for the case of convex co-compact quotients)

FUP holds with exponent $\gamma \Longrightarrow \forall \varepsilon>0 \quad \operatorname{Spec}\left(B_{N}\right) \cap\left\{|\lambda| \geq M^{-\gamma+\varepsilon}\right\}=\emptyset$, for $N>N_{\varepsilon}$,

The case of $\gamma=\frac{1}{2}-\delta$ is easy to establish but a finer analysis of FUP gives [74]:

Theorem 11 Suppose that $B_{N}$ is a quantization of $\kappa_{M, \mathcal{A}}$ and $\delta$ is given in (3.39). Then there exists

$$
\beta>\max \left(0, \frac{1}{2}-\delta\right)
$$

such that

$$
\forall \varepsilon>0 \quad \operatorname{Spec}\left(B_{N}\right) \cap\left\{|\lambda| \geq M^{-\beta+\varepsilon}\right\}=\emptyset, \quad N>N_{\varepsilon} .
$$

A numerical illustration of Theorem 11 is shown in Fig. 23. In the example presented there the numerically computed best exponent in FUP (3.43) is sharp. Some other examples in [74] show however that it is not always the case. We will return to open quantum maps in Sect. 3.4.

Theorem 11 is the strongest gap result for hyperbolic trapped sets and it suggests that a resonance free strip of size $h$ exists for all operators $P(h)$ with such trapped sets: 
Conjecture $3^{11}$ Suppose that $P(h)$ given by (3.20) has a compact hyperbolic trapped set $K_{E}$ in the sense of Definition 4 (and $M$ is a manifold for which we have an effective meromorphic continuation of $\left.(P(h)-z)^{-1}\right)$. Then there exists $\gamma>0, \delta>0$ and $h_{0}$ such that

$$
\operatorname{Res}(P(h)) \cap([E-\delta, E+\delta]-i h[0, \gamma])=\emptyset, \quad h<h_{0} .
$$

\subsection{Resonance expansions in general relativity}

In this section we will describe some recent results concerning expansions of solutions to wave equations for black hole metrics in terms of quasi-normal modes (QNM). That is the name by which scattering resonances go in general relativity [157]. We want to emphasize the connection to the results of Sects. 3.1, 3.2 and in particular to the $r$-normally hyperbolic dynamics [274].

We will consider the case of Kerr-de Sitter, or asymptotically Kerr-de Sitter metrics. These model rotating black holes in the case of positive cosmological constant $\Lambda>$ 0 . From the mathematical point of view that makes infinity "larger" and provides exponential decay of waves which makes a rigorous formulation of expansions easier. When one adds frequency localization weaker expansions are still possible in the Kerr case-see [68, Theorem 2], [76, (13)]. References to the extensive mathematics literature in the case of $\Lambda=0$ can be found in [68].

To define the Kerr-de Sitter metric we consider the manifold $X=\left(r_{+}, r_{C}\right) \times \mathbb{S}^{2}$, where $r_{+}$is interpreted as the event horizon of the black hole and $r_{C}$ the cosmological horizon. The wave equation is formulated on $\mathbb{R} \times X$ using a Hamiltonian defined on $T^{*}(\mathbb{R} \times X)$. We denote the coordinates on $\mathbb{R} \times X$ by $(t, r, \theta, \varphi)$ and write $\left(\xi_{t}, \xi_{r}, \xi_{\theta}, \xi_{\varphi}\right)$ for the corresponding conjugate (momentum) variables. In this notation the Kerr-de Sitter Hamiltonian has the following frightening form:

$$
\begin{aligned}
G & =\rho^{-2}\left(G_{r}+G_{\theta}\right), \quad \rho^{2}=r^{2}+a^{2} \cos ^{2} \theta \\
G_{r} & =\Delta_{r} \xi_{r}^{2}-\frac{(1+\alpha)^{2}}{\Delta_{r}}\left(\left(r^{2}+a^{2}\right) \xi_{t}+a \xi_{\varphi}\right)^{2}, \\
G_{\theta} & =\Delta_{\theta} \xi_{\theta}^{2}+\frac{(1+\alpha)^{2}}{\Delta_{\theta} \sin ^{2} \theta}\left(a \sin ^{2} \theta \xi_{t}+\xi_{\varphi}\right)^{2}, \quad \alpha=\frac{\Lambda a^{2}}{3} . \\
\Delta_{r} & =\left(r^{2}+a^{2}\right)\left(1-\frac{\Lambda r^{2}}{3}\right)-2 M r, \quad \Delta_{\theta}=1+\alpha \cos ^{2} \theta .
\end{aligned}
$$

Here $\Lambda>0$ is the cosmological constant, $a$ is the rotation speed ( $a=0$ corresponds to the simpler case of the Schwarzschild-de Sitter metric), and $M>0$ is a parameter

\footnotetext{
11 After this survey first appeared Conjecture 3 was proved in the case of finitely generated hyperbolic surfaces by Bourgain-Dyatlov [34]. That means that for convex co-compact surfaces, $\Gamma \backslash \mathbb{H}^{2}$ —see Fig. 19 and [31] - there is a high energy gap for all values, $0 \leq \delta<1$, of the dimension of the limit set of $\Gamma-$ see (3.31). This is the first result about gaps for quantum Hamiltonians for any value of the pressure $\mathcal{P}_{E}\left(\frac{1}{2}\right)$ defined in (3.29). The proof is based on the fractal uncertainty principle of [75] and fine harmonic analysis estimates related to the Beurling-Malliavin multiplier theorem, see [175] and references given there.
} 

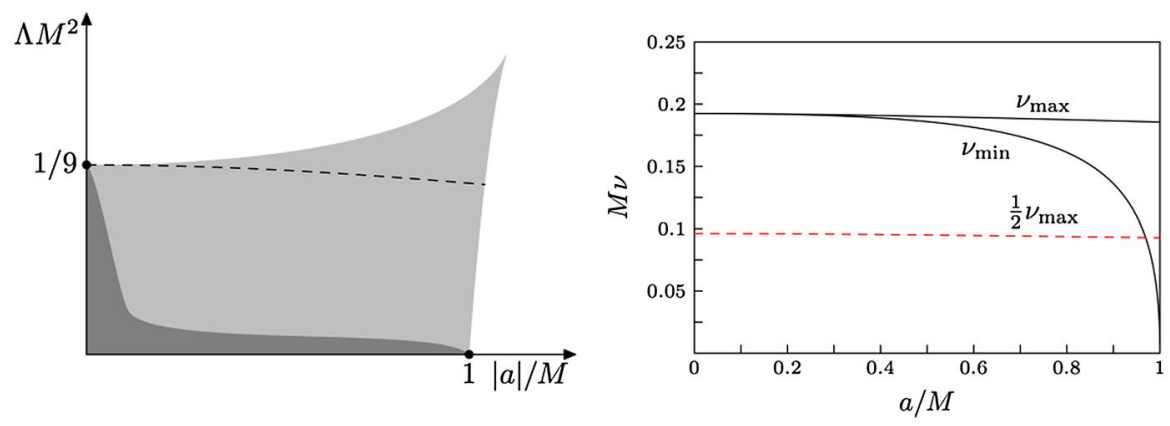

Fig. 24 Left numerically computed admissible range of parameters for the subextremal Kerr-de Sitter black hole (light shaded) and the range to which the resonance expansions of [68] apply. QNM are defined and discrete for parameters below the dashed line, $\left(1-\Lambda a^{2} / 3\right)^{3}=9 \Lambda M^{2}$, see [68, §3.2]. Right The dependence of $v_{\max }$ and $v_{\min }$ on the parameters $M$ and $a$ in the case of $\Lambda=0$. The dashed line indicates the range of validity of the pinching condition needed for the Weyl law [67] recalled in Fig. 22

(in the case of $\Lambda=0$ it is the mass of the black hole)-see Fig. 24 for the allowed range of parameters. The Hamiltonian $G$ is the dual metric to the semi-Riemannian Kerr-de Sitter metric $g$. The principal symbol of the wave operator $\square_{g}$ is given by $G$. We refer to Hintz [127] and Hintz-Vasy [129] for a geometric description of these metrics and definitions of asymptotically Kerr-de Sitter metrics.

The key fact is that $\Delta_{r}$ has simple zeros at $r=r_{+}$and $r=r_{C}$ which gives

$$
P_{g}(\lambda):=e^{-i \lambda t} \square_{g} e^{i \lambda t}: \mathcal{C}^{\infty}(X) \rightarrow \mathcal{C}^{\infty}(X), \quad X=\left(r_{+}, r_{C}\right) \times \mathbb{S}^{2},
$$

structure similar to that of $P(\lambda)$ in (3.12), with $r=r_{+}$or $r=r_{C}$ corresponding to $x_{1}=0$ (when $a=0, r \in\left(r_{+}, r_{C}\right)$ corresponds to $x_{1}>0$; for rotating black holes the situation is more complicated but microlocally the structure is similar).

Since we are interested in solving $\square_{g} w=0$ we consider the null geodesic flow $\varphi_{t}:=\exp t H_{G}$ (see 3.23) on the positive light cone $\mathcal{C}_{+} \subset\{G=0\}$. (Null stands for $G=0$ which is preserved by the flow.) The trapped set, $\mathcal{K}$, consists of null geodesics that stay away from $r=r_{+}, r=r_{C}$ for all times. We refer to Dyatlov [68, Prop.3.2] for the description of this trapped set and the constraints on the parameters $a, M$ and $\Lambda$. Here we remark that it has a particularly simple form when $a=0$ :

$$
\mathcal{K}=\left\{\left(t, 3 M, \omega ; \xi_{t}, 0, \omega^{*}\right):\left(\omega, \omega^{*}\right) \in T^{*} \mathbb{S}^{2}, \frac{1-9 \Lambda M^{2}}{27 M^{2}}\left|\omega^{*}\right|_{\omega}^{2}=\xi_{t}^{2}\right\},
$$

where $|\bullet|_{\omega}$ is the standard metric on $T_{\omega}^{*} \mathbb{S}^{2}$.

In general $\mathcal{K}$ is symplectic in the sense that the symplectic form on $T^{*} X$ is nondegenerate on the surfaces $K=\mathcal{K} \cap\{t=$ const $\}$. The flow is $r$-normally hyperbolic for any $r$. That means that there exists a $C^{r}$ splitting $T_{\mathcal{K}} \mathcal{C}_{+}=T \mathcal{K} \oplus \mathcal{V}_{+} \oplus \mathcal{V}_{-}$, invariant under the flow and such that for some constants $v>0, C>0$,

$$
\left.\sup _{(x, \xi) \in K}\left|d \varphi^{\mp t}\right|_{\mathcal{V}_{ \pm}}\left|\leq C e^{-v t}, \quad \sup _{(x, \xi) \in K}\right| d \varphi^{ \pm t}\right|_{T K} \mid \leq C e^{v|t| / r}, t \geq 0
$$


In other words, the maximal expansion rates (Lyapunov exponents) on the trapped set are $r$-fold dominated by the expansion and contraction rates in the directions transversal to the trapped set. As shown in Hirsch-Pugh-Schub [132] (see also [68, §5.2]) $r$-normal hyperbolicity is stable under perturbations: when $G_{\varepsilon}$ is a time independent (that is, stationary) Hamiltonian such that $G_{\varepsilon}$ is close to $G$ in $C^{r}$ near $\mathcal{K}$, the flow for $G_{\varepsilon}$ is $r$-normally hyperbolic in the sense that the trapped set $\mathcal{K}_{\varepsilon}$ has $C^{r}$ regularity, is symplectic and (3.48) holds. For Kerr(-de Sitter) metrics the flow is $r$-normally hyperbolic for all $r$ as shown by Wunsch-Zworski [274], Vasy [263] and Dyatlov [68], essentially because the flow on $\mathcal{K}$ is completely integrable. The dependence of maximal and minimal expansion rates on the parameters is shown in Fig. 24.

Vasy's method [263] which was described in Sect. 3.1 applies in this setting. Quasinormal modes (QNM) for Kerr-de Sitter metrics are defined as in (3.18): they are complex frequencies $\lambda$ such that there exist $u=u(r, \theta, \phi)$ such that

$$
\square_{g}\left(e^{-i \lambda t} u\right)=0 \text { and } u \text { continues smoothly across the two horizons. }
$$

The connection with (3.18) comes from (3.47) and the fact that $P_{g}(\lambda) u=0$. The same definition works for perturbations.

For Schwarzschild-de Sitter black holes, QNM were described by Sá BarretoZworski [231]: at high frequencies they lie on a pseudo-lattice as in the case of one hyperbolic closed orbit [107] but with multiplicities coming from the spherical degeneracy. Dyatlov $[66,67]$ went much further by describing QNM for Kerr-de Sitter black holes: for small values of rotations he showed a Zeeman-like splitting of multiplicities predicted in the physics literature, and for perturbations of Kerr-de Sitter black holes, he obtained a counting law (see Figs. 22, 24). In particular, for small values of $a$ the results of [66] show that there are no modes with $\operatorname{Im} \lambda \geq 0$. For Kerr black holes ((3.46) with $\Lambda=0$ ) Shlapentokh-Rothman [238] showed that this is the case in the full range $|a|<M$. This suggests the following

Conjecture 4 For $a, \Lambda$ and $M$ satisfying $\left(1-\Lambda a^{2} / 3\right)^{3}>9 \Lambda M^{2}$ and in the shaded region of Fig. 24 (or in some other "large" range of values), any $\lambda \neq 0$ for which (3.49) holds satisfies $\operatorname{Im} \lambda<0$ and $\lambda=0$ is a simple resonance.

The study of QNM is motivated by the expectation that forward solutions to $\square_{g} u=$ $f \in \mathcal{C}_{\mathrm{c}}^{\infty}(\mathbb{R} \times X)$ have expansions in terms of $u_{k}$ 's and $\lambda_{k}$ 's satisfying (3.49),

$$
u(t, r, \theta, \varphi) \sim \sum_{k} e^{-i \lambda_{k} t} u_{k}(r, \theta, \varphi),
$$

but the convergence and errors can be subtle.

The first expansion involving infinitely many QNM lying on horizontal strings of the pseudo-lattice of [231] was obtained by Bony-Häfner [29]. Using his precise results on the distribution of QNM for Kerr-de Sitter metrics with small values of $a$, Dyatlov [66] obtained similar expansions for rotating black holes. In [67] he formulated an expansion of waves for perturbations of Kerr-de Sitter metrics in terms of a microlocal projector, see also [68]. 
Here we will only state a simpler result which is an almost immediate consequence of Theorems 8, 10 and of gluing results of Datchev-Vasy [57]. For tensor-valued wave equations on perturbations of Schwarzschild-de Sitter spaces (including Kerr-de Sitter spaces with small values of $a$ ) and in any space-time dimension $n \geq 4$ this result was obtained by Hintz [127]. A more precise formulation valid across the horizons can be found there.

Theorem 12 Suppose that $(X, g)$ is a stationary perturbation of the Kerr-de Sitter metric in the sense of [127,129] and that $u$ is the forward solution $\square_{g} u=f \in$ $\mathcal{C}_{\mathrm{c}}^{\infty}(\mathbb{R} \times X)$. Let $v_{\min }$ be the minimal expansion rate at the trapped set given by (3.36) (see Fig. 24). Then for any $\varepsilon>0$,

$$
u(t, x)=\sum_{\operatorname{Im} \lambda_{j}>-\frac{1}{2} v_{\min }+\varepsilon} \sum_{m=0}^{m_{j}} \sum_{\ell=0}^{d_{j}} t^{\ell} e^{-i \lambda_{j} t} u_{j m \ell}(x)+\mathcal{O}_{K}\left(e^{-\left(v_{\min } / 2-\varepsilon\right) t}\right), x \in K \Subset X .
$$

When $d_{j}=0, u_{j, m, 0}$ and $\lambda_{j}$ satisfy (3.49); otherwise the resonance expansion is computed from the residue of $\lambda \mapsto P_{g}(\lambda)^{-1} e^{-i \lambda t}$ - see $[129, \S 5.1 .1]$ (and [80, Theorem 2.7] for a simple example).

\subsection{Upper bounds on the number of resonances: fractal Weyl laws}

The standard Weyl law for the density of quantum states was already recalled in (2.29) as motivation for the upper bound on the number of resonances in discs (2.26). In this section we will describe finer upper bounds which take into account the geometry of the trapped set. They were first proved by Sjöstrand [240] for operators with analytic coefficients but in greater geometric generality than presented here.

Throughout this section we assume that

$$
P(h) \text { given by (3.20) has a compact hyperbolic trapped set } K_{E}
$$

in the sense of Definition 4 and that $M$ is a manifold for which we have an effective meromorphic continuation of $(P(h)-z)^{-1}$ (see Sects. 2.6, 3.1).

When $M$ is a compact manifold then $P(h)$ has discrete spectrum and the counting laws have a long tradition-see Ivrii [144]. The basic principle can be described as follows: if we are interested in

$$
N_{P(h)}([a, b]):=|\operatorname{Spec}(P(h)) \cap[a, b]|, \quad P(h)=-h^{2} \Delta_{g}+V,
$$

then the relevant region in phase space is given by

$$
p^{-1}([a, b])=\{(x, \xi): a \leq p(x, \xi) \leq b\}, \quad p(x, \xi):=|\xi|_{g}^{2}+V(x)
$$

The uncertainty principle says that a maximal (homogeneous) localization of a state in phase space is to a box of sides of length $\sqrt{h}$ (here centered at $(0,0))$ : 


$$
\left(\int_{\mathbb{R}^{n}}\left|x_{j} u(x)\right|^{2} d x\right)^{\frac{1}{2}} \times\left(\int_{\mathbb{R}^{n}}\left|\xi_{j} \mathcal{F}_{h} u(\xi)\right|^{2} d \xi\right)^{\frac{1}{2}} \geq \frac{h}{2}\|u\|_{L^{2}}^{2},
$$

where $\mathcal{F}_{h} u(\xi):=(2 \pi h)^{-n / 2} \int_{\mathbb{R}^{n}} u(x) e^{-i\langle x, \xi\rangle / h} d x$ is the (unitary) semiclassical Fourier transform. Hence the maximal number of quantum states "fitting" into an $h$-independent open set $U \subset T^{*} M$ is proportional to $\operatorname{vol}(U) h^{-n}$. For eigenvalue counting we need a factor of $(2 \pi)^{-n}$ which gives

$$
N_{P(h)}([a, b])=(2 \pi h)^{-n}\left(\operatorname{vol}\left(p^{-1}([a, b])\right)+o(1)\right) .
$$

By rescaling and taking $[a, b]=[0,1]$ we obtain (2.29).

Suppose now that we consider a scattering problem, in which case $M$ is noncompact, say, equal to $\mathbb{R}^{n}$ outside a compact set. In that case for a subset $\Omega=\Omega(h)$ we can define the number of resonances of $P(h)$ in $\Omega$ :

$$
N_{P(h)}(\Omega)=|\operatorname{Res}(P(h)) \cap \Omega|,
$$

which generalizes the definition above. The basic heuristic principle is that the trapped set $K_{\Omega \cap \mathbb{R}}$ should replace $p^{-1}([a, b])$ as everything else escapes. To guarantee that escape we assume that $K \subset\{\operatorname{Im} z>-\gamma h\}$. This restriction on the imaginary parts is explained by the fact that the corresponding resonant states have an $h$-independent rate of decay: $\operatorname{Re} e^{-i t z / h}=e^{t \operatorname{Im} z / h} \geq e^{-t \gamma}$.

We should then look at the maximal number of localized quantum states that can cover $K_{\Omega \cap \mathbb{R}}$. But that number is now related to the upper box or upper Minkowski dimension of $K_{\Omega \cap \mathbb{R}}$ :

$$
\operatorname{dim}_{M}^{+}(U):=\limsup _{h \rightarrow 0} \frac{n(\sqrt{h}, U)}{\log (1 / \sqrt{h})}
$$

where $n(\varepsilon, U)$ is the minimal number of boxes of sides $\varepsilon$ needed to cover $U$. This suggests that we should have an upper bound

$$
N_{P(h)}([a, b]-i h[0, \gamma]) \leq C h^{-\frac{1}{2} \operatorname{dim}_{M}^{+}\left(K_{[a, b]}\right)} .
$$

This intuition is put to test by the fact that resonant states with $\operatorname{Im} z>-\gamma h$ are not localized to the trapped set but rather to the outgoing tails $\Gamma_{E}^{+}$given in (3.24) - see Fig. 25 and [200, Theorem 4]. The method of complex scaling described in Sect. 2.6 changes the exponential growth we see in Fig. 25 to exponential decay and hence localizes the resonant state to the interaction region. By a microlocal complex scaling constructed using suitable low regularity microlocal weights Sjöstrand [240] similarly localized resonant states to an $\sqrt{h}$ neighbourhood of the trapped set and hence was able to prove bounds of the form (3.54). Although we concentrate here on hyperbolic trapped sets we remark that Dyatlov's asymptotic formula [67] in Fig. 22 is consistent with (3.54).

Put

$$
d_{E}:=\frac{\operatorname{dim}_{M}^{+} K_{E}-1}{2}, \quad \delta_{E}=\frac{\operatorname{dim}_{H} K_{E}-1}{2},
$$



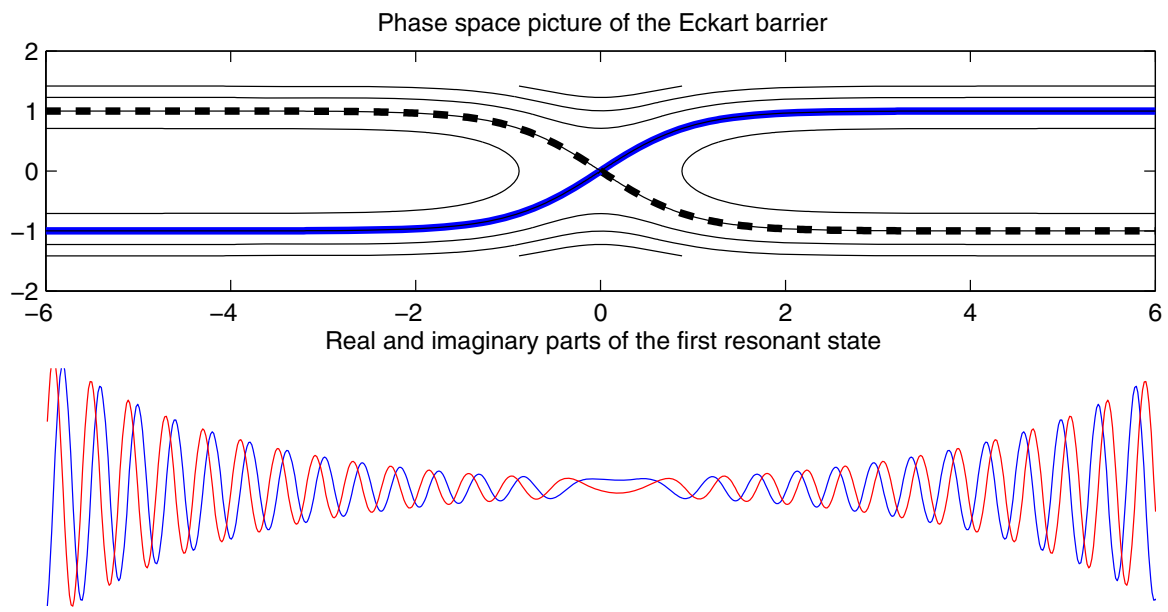

Density plot of the FBI transform of the first resonant state

Fig. 25 The top figure shows the phase portrait for the Hamiltonian $p(x, \xi)=\xi^{2}+\cosh ^{-2}(x)$, with $\Gamma_{1}^{ \pm}$ highlighted. The middle plot shows the resonant state corresponding to the resonance closest to the real axis at $h=1 / 16$ (compare this to the resonances in Fig. 9 generated by the local maxima), and the bottom plot shows the squared modulus of its FBI tranform (which describes a wave function in phase space [281, Chapter 13]). The resonance states were computed by D. Bindel [21] and the FBI transform was provided by L. Demanet. We see that the mass of the FBI transform is concentrated on $\Gamma_{1}^{+}$, with the exponential growth in the outgoing direction. See also https://www.youtube.com/watch?v=R7eUpEVpckk\&feature=youtu.be\& list=PL32oQ9j1OWc0RIINwnY6BtDawepnBnQyx

where $\operatorname{dim}_{H}$ is the Hausdorff dimension (hence $\delta_{E} \leq d_{E}$ ). Then the following theorem was proved for Euclidean infinities by Sjöstrand-Zworski [248], for even asymptotically hyperbolic infinities (see Sect. 3.1) by Datchev-Dyatlov [53] (generalizing earlier results $[122,280])$ and for several convex obstacles by Nonnenmacher-SjöstrandZworski [198]:

Theorem 13 Under the assumption (3.50) and using the notation (3.53),(3.55), for every $\gamma$ and $\varepsilon>0$ there exists $C$ such that

$$
N_{P(h)}([E-h, E+h]-i h[0, \gamma]) \leq C h^{-d_{E}-\varepsilon} .
$$

When $V=0$ in (3.20) and $\operatorname{dim} M=2$ we can replace $d_{E}+\varepsilon$ by $\delta_{E}$ in (3.56).

For outlines and ideas of the proofs we refer the reader to general reviews [196, $\S \S 4,7],[193]$ and to [248, §2], [53, §1], [198, §1].

We remark that a fractal upper bound for resonances should be valid for more general quotients $\Gamma \backslash \mathbb{H}^{n}$ than the ones considered in [53]—see Guillarmou-Mazzeo 

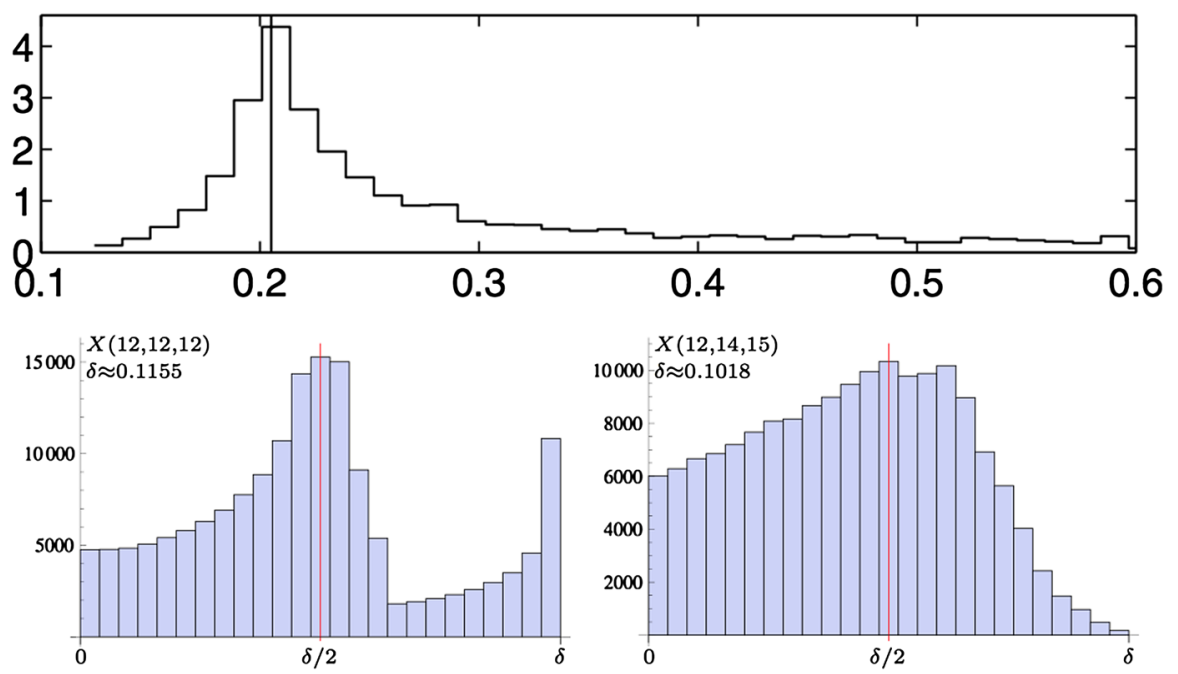

Fig. 26 Top histogram of imaginary parts of resonances of a three discs configuration (see Fig. 2) with real parts with $|\operatorname{Re} \lambda| \leq 2 \times 10^{3}$ computed using a semiclassical zeta function [170]. The imaginary parts concentrate at $\frac{1}{2} \mathcal{P}_{1}(1)$, that is at one half of the classical escape rate. (We should remark here that the method of calculation based on the zeta function, although widely accepted in the physics literature, does not have a rigorous justification and may well be inaccurate. However, the phenomenon of concentration at $\frac{1}{2} \mathcal{P}_{1}(1)$ has been verified experimentally—see [13] and Fig. 10). Bottom histograms from Borthwick [30] showing the distribution of imaginary parts (the $x$ axis is $\left.\operatorname{Im} \lambda+\frac{1}{2}\right)$ for hyperbolic surfaces $X\left(\ell_{1}, \ell_{2}, \ell_{3}\right)$ (see Fig. 19) with $|\operatorname{Re} \lambda| \leq 2 \times 10^{4}$. The concentration at $\operatorname{Im} \lambda=\frac{1}{2}(\delta-1)$ is clearly visible. Recalling (3.27) and (3.31) that is the same as in the top figure

[119] for results on meromorphic continuation in that case. Even the simplest case of surfaces with both funnels and cusps is not understood (the only results treat the non-trapping case-see Datchev [52] and Datchev-Kang-Kessler [56]).

Existence of fractal lower bounds or asymptotics has been studied numerically, first for a three bump potential by Lin [167] and then using semiclassical zeta function calculations [103] for three disc systems (see Figs. 2 and 10) by LuSridhar-Zworski [170]. The results have been encouraging and led to experimental investigation by Potzuweit et al [216]: the experimentally observed density is higher than linear but does not fit the upper bound (3.56). There are many possible reasons for this, including the limited range of frequencies. (Related experiments by Barkhofen et al [13] confirmed the pressure gaps of Theorem 9, see Figs. 10 and 26). Fractal Weyl laws have been considered (and numerically checked) for various open chaotic quantum maps—see [74,199] and references given there. The have also been proposed in other types of chaotic systems, ranging from dielectric cavities to communication and social networks - see recent review articles by CaoWiersig [42] and Ermann-Frahm-Shepelyansky [83] respectively. Fractal Weyl laws for mixed systems and localization of resonant states have been investigated by Körber et al $[158,159]$.

So far, the only rigorous lower bound which agrees with the fractal upper bound was obtained by Nonnenmacher-Zworski in a special toy model quantum map [200, 
Theorem 1]. Nevertheless the evidence seems encouraging enough to reiterate the following conjecture [170], [123, (4.7)]:

Conjecture 5 Suppose that $N(\Omega):=N_{P(h)}(\Omega)$ is defined in $(3.53)$ and $\delta_{E}$ is given by (3.55). If $\operatorname{dim} M=2$, then

$$
\lim _{h \rightarrow 0} h^{\delta_{E}} N([E-h, E+h]-i h[0, \gamma])=v_{E}(\gamma)
$$

where $v_{E}(\gamma)>0$ for $\gamma$ large enough. When $\operatorname{dim} M>2$ then

$$
\limsup _{h \rightarrow 0} h^{\delta_{E}} N([E-h, E+h]-i h[0, \gamma])>0
$$

when $\gamma$ is large enough.

Numerical investigation of fractal Weyl laws lead to the observation that imaginary parts of resonances (that is resonance widths) concentrate at $\operatorname{Im} z \approx \frac{1}{2} \mathcal{P}_{E}(1) h$ where $\mathcal{P}_{E}(s)$ is defined by (3.29). The value $\left|\mathcal{P}_{E}(1)\right|$ gives the classical escape rate on the energy surface $p^{-1}(E)$-see [196, (17)]. The point made by Gaspard-Rice [103] was that the gap is given by $\left|\mathcal{P}_{E}\left(\frac{1}{2}\right)\right| h<\frac{1}{2}\left|\mathcal{P}_{E}(1)\right| h$ (with the latter being a more obvious guess). However most of resonances want to live near $\operatorname{Im} z=\frac{1}{2} \mathcal{P}_{E}(1) h$-see also Fig. 21.

The first rigorous result indicating lower density away from $\operatorname{Im} \lambda=\frac{1}{2} \mathcal{P}_{E}(1)$ was obtained by Naud [192] who improved on the fractal bound in Theorem 13 for $\gamma<\left|\mathcal{P}_{E}(1)\right| / 2$ in the case of convex co-compact surfaces. Dyatlov [70] provided an improved bound for convex co-compact hyperbolic quotients in all dimensions. We state his result in the case of dimension two and in the non-semiclassical setting (resonances as poles of $\left(-\Delta_{g}^{2}-\frac{1}{4}-\lambda^{2}\right)^{-1}-$ see (3.7), (3.27) and (3.31)):

Theorem 14 Let $M=\Gamma \backslash \mathbb{H}^{2}$ be a convex co-compact quotient (see Fig. 19) and define $\mathcal{N}(r, \beta)$ to be the number of poles of the continuation of $\left(-\Delta_{g}-\frac{1}{2}-\lambda^{2}\right)^{-1}$ in

$$
r<\operatorname{Re} \lambda \leq r+1, \quad \operatorname{Im} \lambda>-\frac{1}{2}+(1-\beta) \delta
$$

where $\delta$ is defined in (3.31). Then for any $\beta$ and $\varepsilon>0$ there exists $C$ such that

$$
\mathcal{N}(r, \beta) \leq C r^{\min (2 \beta \delta+\varepsilon, \delta)} .
$$

The bound $r^{\delta}$ comes from [122] and the improvement is in strips $\operatorname{Im} \lambda>-\gamma>$ $-\frac{1}{2}(1-\delta)$. A comparison between (3.59) and numerically fitted exponents was shown in Fig. 21 (for the more robust counting fuction $N(R, \beta)=\sum_{k=1}^{[R]} \mathcal{N}(R-k, \beta)$ ). These numerical experiments of Borthwick-Dyatlov-Weich [70, Appendix] again suggest that Conjecture 5 (perhaps in a weaker form) holds, while the optimality of (3.59) is unclear. Some lower bounds in strips have been obtained by Jakobson and Naud [146] but they are far from the upper bounds. 
Dyatlov-Jin [74] proved an analogue of Theorem 14 for open quantum maps of the form shown in Fig. 23. That paper can be consulted for more numerical experiments and interesting conjectures.

Here we make a weak (and hopefully accessible) conjecture which in the case of quotients follows from Theorem 14:

Conjecture 6 In the notation of (3.57) and for general operators satisfying (3.50)

$$
\gamma<\frac{1}{2}\left|\mathcal{P}_{E}(1)\right| \Longrightarrow v_{E}(\gamma)=0 \text {. }
$$

Jakobson and Naud [147] made a bolder conjecture that for convex co-compact hyperbolic surfaces there are only finitely many resonances with $\operatorname{Im} \lambda>-\frac{1}{2}(1-\delta)-$ see Fig. 21. We make an equally bold but perhaps more realistic

Conjecture 7 Suppose that $N(\Omega):=N_{P(h)}(\Omega)$ is defined in (3.53). Then for every $\gamma>\mathcal{P}_{E}(1) / 2$, there exists $\varepsilon_{0}>0$ such that for every $\varepsilon<\varepsilon_{0}$,

$$
\lim _{h \rightarrow 0} \frac{N\left([E-h, E+h]-\frac{1}{2} i h\left[\mathcal{P}_{E}(1)-\varepsilon, \mathcal{P}_{E}(1)+\varepsilon\right]\right)}{N([E-h, E+h]-i h[0, \gamma])}=1,
$$

where $\mathcal{P}_{E}(s)$ is the topological pressure defined in (3.29).

In the case of convex co-compact hyperbolic quotients Theorem 14 and Conjecture 5 imply that, for $\gamma$ large enough,

$$
\lim _{h \rightarrow 0} \frac{N\left([E-h, E+h]-\frac{1}{2} i h\left[0, \mathcal{P}_{E}(1)-\varepsilon, \gamma\right]\right)}{N([E-h, E+h]-i h[0, \gamma])}=1 .
$$

That could be a weaker but already very interesting substitute for (3.60).

\section{Pollicott-Ruelle resonances from a scattering theory viewpoint}

Suppose that $M$ is a manifold and $X$ a smooth vector field on $M$. The flow $\varphi_{t}:=\exp t X$ gives a group of diffeomorphisms $\varphi_{t}: M \rightarrow M$ and a large branch of the theory of dynamical systems is concerned with long time properties of the evolution under $\varphi_{t}$. One way to measure this evolution is by considering correlations: for $f, g \in \mathcal{C}_{\mathrm{c}}^{\infty}(M)$ we define

$$
\rho_{f, g}(t):=\int_{M} f\left(\varphi_{-t}(x)\right) g(x) d m(x),
$$

with respect to a measure on $M$. (Sometimes $\left(\int_{M} f\right) \times\left(\int_{M} g\right)$ is subtracted from $\rho_{f, g}$ in the definition; it also interesting to consider less regular $f$ and $g$ ). What measure one should take is of great interest and invariant measures are the most natural ones. The particularly interesting Sinai-Ruelle-Bowen (SRB) measures (see [154]) have the property that

$$
\rho_{f, g}(t)=\lim _{T \rightarrow \infty} \frac{1}{T} \int_{0}^{T} f\left(\varphi_{s-t}(x)\right) g\left(\varphi_{s}(x)\right) d s
$$


for almost every $x \in M$ with respect to a smooth (Lebesgue) measure. This allows a computation of $\rho_{f, g}(t)$ using one "randomly chosen" orbit of the flow.

With $U(t) f:=f \circ \varphi_{-t}$ this is the set-up discussed in Fig. 3 and we expect that for chaotic flows we have a picture shown there. That is, we expect an expansion of correlations in terms of suitably defined resonances of the dynamical systems,

$$
\rho_{f, g}(t) \sim \sum_{\operatorname{Im} \lambda_{j}>-\gamma} e^{-i \lambda_{j} t} u_{j}(f) v_{j}(g)+\mathcal{O}_{f, g}\left(e^{-\gamma t}\right),
$$

where $u_{j}, v_{j} \in \mathcal{D}^{\prime}(M)$ and $\lambda_{j}$ are independent of the test functions $f$ and $g$. If we know that the number of $\lambda_{j}$ 's with $\operatorname{Im} \lambda_{j}>-\gamma$ is finite and $\lambda_{0}=0$ (with $u_{0}=v_{0}=d \mathrm{~m}$ ) is the only real resonance then we have an exponential decay of correlations:

$$
\rho_{f, g}(t)-\int_{M} f(x) d m(x) \int_{M} g(x) d m(x)=\mathcal{O}\left(e^{-\alpha t}\right) .
$$

Hence we see that the questions encountered here are similar to the questions asked in the study of scattering resonances: existence of resonance expansions (Sects. 2.5, 3.3) and of resonance free regions (gaps) (Sects. 2.4, 3.2). One can also ask general questions about the distribution of $\lambda_{j}$ 's.

As signatures of a chaotic systems the dynamical resonances $\lambda_{j}$ 's were introduced by Pollicott [213] and Ruelle [227]—see Fig. 13 for an example in modeling of physical phenomena. They can also be studied in the simpler setting of maps, that is for systems with discrete time-see Baladi-Eckmann-Ruelle [8] for an early study and Baladi $[5,6]$ for later developments.

The explicit analogy with scattering theory was emphasized by Faure-Sjöstrand [84] following earlier works by Faure-Roy-Sjöstrand [86] and Faure-Roy [85] in the case of maps. Semiclassical methods for the study of decay of correlations were also introduced by Tsujii [261] who applied FBI transform techniques to obtain precise bounds on the asymptotic gaps for certain flows (see Sect. 4.4 below). This led to rapid progress some of which is described below-to see the extent of this progress one can compare the current state of affairs to that in the early review by Eckmann [81]. For some other recent developments see also Faure-Tsujii [89].

In this section we first give a precise definition of chaotic dynamical systems and then define Pollicott-Ruelle resonances. We concentrate to the case of $M$ compact but give indications what happens in the non-compact case. We then explain the connection to dynamical zeta functions and survey results on resonance free strips and on ounting of resonances.

\subsection{Anosov dynamical systems}

Let $M$ be a compact manifold and $\varphi_{t}=\exp t X: M \rightarrow M$ a $C^{\infty}$ flow generated by $X \in C^{\infty}(M ; T M)$.

In this article the precise meaning of being chaotic is that the flow is an Anosov flow. That means that the tangent space to $M$ has a continuous decomposition 
$T_{x} M=E_{0}(x) \oplus E_{s}(x) \oplus E_{u}(x)$ which is invariant, $d \varphi_{t}(x) E_{\bullet}(x)=E_{\bullet}\left(\varphi_{t}(x)\right)$, $E_{0}(x)=\mathbb{R} X_{x}$, and for some $C$ and $\theta>0$ fixed

$$
\begin{aligned}
& \left|d \varphi_{t}(x) v\right|_{\varphi_{t}(x)} \leq C e^{-\theta|t|}|v|_{x}, \quad v \in E_{u}(x), \quad t<0 \\
& \left|d \varphi_{t}(x) v\right|_{\varphi_{t}(x)} \leq C e^{-\theta|t|}|v|_{x}, \quad v \in E_{S}(x), \quad t>0
\end{aligned}
$$

where $|\bullet|_{y}$ is given by a smooth Riemannian metric on $X$. This should be compared to Definition 4: in the case when $M$ is compact all of $M$ is "trapped" and Anosov flows are hyperbolic everywhere.

Following Faure-Sjöstrand [84] we exploit the analogy between dynamical systems and quantum scattering, with the fiber $\xi$-infinity playing the role of $x$-infinity in scattering theory. The pullback map can be written analogously to the Schrödinger propagator:

$$
\begin{aligned}
\varphi_{-t}^{*} & =e^{-i t P}: \mathcal{C}^{\infty}(M) \rightarrow \mathcal{C}^{\infty}(M), \\
\varphi_{-t}^{*} f(x) & :=f\left(\varphi_{-t}(x)\right), \quad P:=\frac{1}{i} X .
\end{aligned}
$$

Sometimes it is convenient to make the seemingly trivial semiclassical modification and write $P_{h}=\frac{h}{i} X, \varphi_{-t}^{*}=e^{-i t P_{h} / h}$. In either case the symbol of $P$ and its Hamiltonian flow

$$
\sigma(P)=: p(x, \xi)=\xi\left(X_{x}\right), e^{t H_{p}}(x, \xi)=\left(\varphi_{t}(x),\left({ }^{T} d \varphi_{t}(x)\right)^{-1} \xi\right) .
$$

Here $H_{p}$ denotes the Hamilton vector field of $p: \omega\left(\bullet, H_{p}\right)=d p$, where $\omega=d(\xi d x)$ is the symplectic form on $T^{*} M$. This should be compared to (3.22) and (3.23), noting that $\varphi_{t}$ mean a different thing in Sect. 3. For us the classical flow $\varphi_{t}$ on $M$ is the "quantum propagation" $e^{-i t P}$ and the corresponding Hamiltonian flow $\exp t H_{p}$ is the symplectic lift of $\varphi_{t}$ to $T^{*} X$ (see Fig. 27).

In the study of $P$ we need the dual decomposition of the cotangent space:

$$
T_{x}^{*} X=E_{0}^{*}(x) \oplus E_{s}^{*}(x) \oplus E_{u}^{*}(x),
$$

where $E_{0}^{*}(x), E_{s}^{*}(x), E_{u}^{*}(x)$ are annhilators of $E_{s}(x) \oplus E_{u}(x), E_{0}(x) \oplus E_{s}(x)$, and $E_{0}(x) \oplus E_{u}(x)$. Hence they are dual to to $E_{0}(x), E_{u}(x), E_{s}(x)$, respectively.

An important special class of Anosov flows is given by contact Anosov flows. In that case $M$ is a contact manifold, that is a manifold equipped with a contact 1-form $\alpha$ : that means that if the dimension of $M$ is $2 k+1$ then $(d \alpha)^{\wedge k} \wedge \alpha$ is non-degenerate. A contact flow is the flow generated by the Reeb vector field $X$ :

$$
\alpha(X)=1, d \alpha(X, \bullet)=0
$$

Natural examples of Anosov contact flows are obtained from negatively curved Riemannian manifolds $(\Sigma, g)$ :

$$
M=S^{*} \Sigma:=\left\{(z, \zeta) \in T^{*} \Sigma:|\zeta|_{g}=1\right\}, \quad \alpha=\left.\zeta d z\right|_{S^{*} \Sigma}
$$



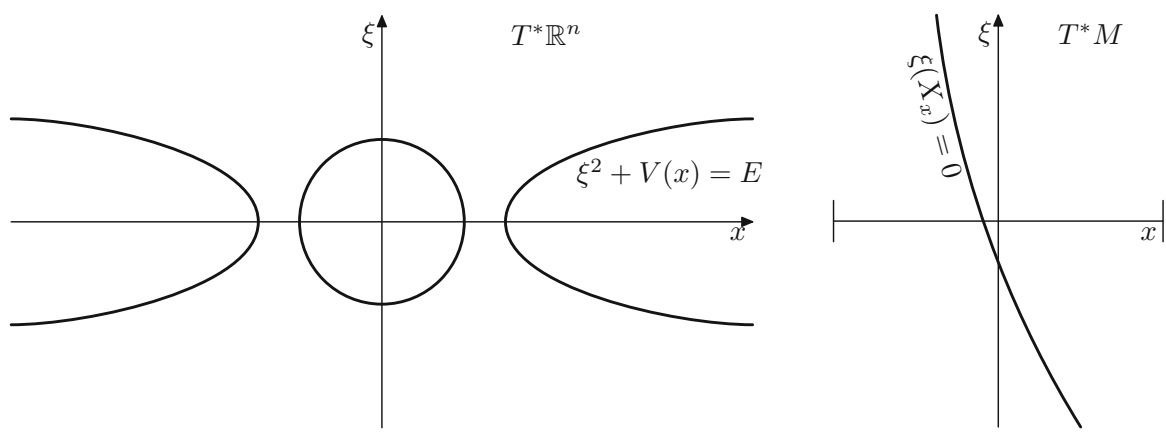

Fig. 27 Scattering in $\mathbb{R}^{n}$ and Anosov flows on a compact manifold $M$ : the classical Hamiltonians in phase space (now a "double phase space" in case of Anosov flow, $T^{*} M$ ) are $p(x, \xi)=|\xi|^{2}+V(x)$, $(x, \xi) \in T^{*} \mathbb{R}^{n}$ and $p(x, \xi)=\xi\left(X_{x}\right),(x, \xi) \in T^{*} M$ (where $X$ generates the Anosov flow). In both cases, the energy surfaces $p=E$ are non-compact: in scattering $x \rightarrow \infty$, in the Anosov case, $\xi \rightarrow \infty$. Fig. 28 below should be compared to [183, Figure 2]: it is not surprising that Melrose's "radial estimates" play a role in the analysis of both cases. For flows the situation is simpler since the flow is linear in the $\xi$ variables (see 4.6) and there is no additional infinity (in the scattering case there is also the $\xi$ infinity). When $M$ is non-compact the situation becomes more complicated as both infinities play a role: see [73, Figure 2]

The scattering theory/microlocal point of view to Anosov flows has already had applications outside the field: by Dang-Riviére [49] to the analysis of Morse-Smale gradient flows, by Guillarmou [116,117], Guillarmou-Monard [120], GuillarmouPaternain-Salo-Uhlmann [121] to inverse problems and by Faure-Tsujii to the study of semiclassical zeta functions [88].

\subsection{Definition of Pollicott-Ruelle resonances}

The intuitive definition should follow Fig. 3. We should consider the power spectrum of correlations:

$$
\hat{\rho}_{f, g}(\lambda):=\int_{0}^{\infty} e^{i \lambda t} \rho_{f, g}(t) d t
$$

which is well defined for $\operatorname{Im} \lambda>0$. If we show that

$$
\lambda \longmapsto \hat{\rho}_{f, g}(\lambda) \text { continues meromorphically to } \operatorname{Im} \lambda>-A,
$$

then the poles of the continuation of $\hat{\rho}_{f, g}(\lambda)$ are the complex frequencies we expect to appear in expansions of correlations (4.2).

Let us assume now that the correlations (4.1) are defined using a smooth (not necessarily invariant) density $\operatorname{dm}(x)$. We denote by $\langle\bullet, \bullet\rangle$ the distributional pairing using this density. In the notation of (4.5) and for $\operatorname{Im} \lambda>0$,

$$
\hat{\rho}_{f, g}(\lambda)=\int_{0}^{\infty}\left\langle e^{-i t P} f, g\right\rangle e^{i \lambda t} d t=\frac{1}{i}\left\langle(P-\lambda)^{-1} f, g\right\rangle
$$


which means that (4.11) follows from

$$
(P-\lambda)^{-1}: \mathcal{C}^{\infty}(M) \rightarrow \mathcal{D}^{\prime}(M) \text { continues meromorphically to } \operatorname{Im} \lambda>-A,
$$

To obtain this meromorphic continuation one wants to find suitable spaces on which $P-\lambda$ is a Fredholm operator. A microlocal construction of such Hilbert spaces was provided by Faure-Sjöstrand [84] but the origins of the method lie in the works on anisotropic Banach spaces by Baladi-Tsujii [9], Blank-Keller-Liverani [23], Butterley-Liverani [41], Gouëzel-Liverani [112], and Liverani [168,169] - see [6] for a recent account in the setting of maps.

Here we will follow a modified approach of [78, $\$ 3.1,3.2]$ where the spaces are defined using microlocal weights. To describe the spaces we denote by $\Psi^{0+}(M)$ the space of pseudodifferential operators of order $\varepsilon$ for any $\varepsilon>0$, with $\sigma: \Psi^{m}(M) \rightarrow$ $S^{m}\left(T^{*} M\right) / S^{m-1}\left(T^{*} M\right)$ denoting the symbol map (see (3.22) where we used the semiclassical symbol, $p=\sigma_{h}(P)$ and [78, Appendix B] for definitions and references). We then put

$$
\begin{aligned}
H_{r G}(M) & :=\exp (-r G(x, D)) L^{2}(M), \quad G \in \Psi^{0+}(M), \\
\sigma(G) & =\left(1-\psi_{0}(x, \xi)\right) m_{G}(x, \xi) \log |\xi|_{g},
\end{aligned}
$$

where $\psi_{0} \in C_{\mathrm{c}}^{\infty}\left(T^{*} M,[0,1]\right)$ is 1 near $\{\xi=0\}, m_{G}(x, \xi) \in C^{\infty}\left(T^{*} M \backslash 0,[-1,1]\right)$ is homogeneous of degree 0 and satisfies (see (4.7))

$$
m_{G}(x, \xi)=\left\{\begin{array}{cc}
1 & \text { near } E_{s}^{*} \\
-1 & \text { near } E_{u}^{*}
\end{array} \quad H_{p} m_{G}(x, \xi) \leq 0, \quad(x, \xi) \in T^{*} M \backslash 0\right.
$$

The existence of such $m_{G}$ is shown in [78, Lemma C.1]. Intuitively, it is clear that it can be done if we look at Fig. 28: the flow lines of $H_{p}$ go from $E_{s}^{*}$ to $E_{u}^{*}$ or towards the zero section. Hence we can have a function which is 1 near $E_{s}^{*}$ and -1 near $E_{u}^{*}$ and decreases along the flow.

The properties of $m_{G}$ and hence of the operator $G(x, D)$ show that for $r \geq 0$,

$$
H^{r}(M) \subset H_{r G}(M) \subset H^{-r}(M) .
$$

Moreover, microlocally near $E_{s}^{*}$ and $E_{u}^{*}$ the space $H_{r G}$ is equivalent to $H^{r}$ and $H^{-r}$ respectively. To explain this rigorously we recall the notion of wave front set, $\mathrm{WF}(u)$, of a distribution $u \in \mathcal{D}^{\prime}\left(\mathbb{R}^{n}\right)$ (since the notion is local, or rather microlocal, the definition works for manifolds as well): for $(x, \xi) \in T^{*} \mathbb{R}^{n} \backslash 0$,

$$
\begin{gathered}
(x, \xi) \notin \operatorname{WF}(u) \\
\text { 立 } \\
\exists \varphi \in \mathcal{C}_{\mathrm{c}}^{\infty}\left(\mathbb{R}^{n}\right), \varphi(x) \neq 0, \varepsilon>0 \forall N \exists C_{N} \\
|\widehat{\varphi u}| \leq C_{N}(1+|\eta|)^{-N}, \quad \eta \in \mathbb{R}^{n} \backslash 0,\left|\frac{\eta}{|\eta|}-\frac{\xi}{|\xi|}\right|<\varepsilon,
\end{gathered}
$$




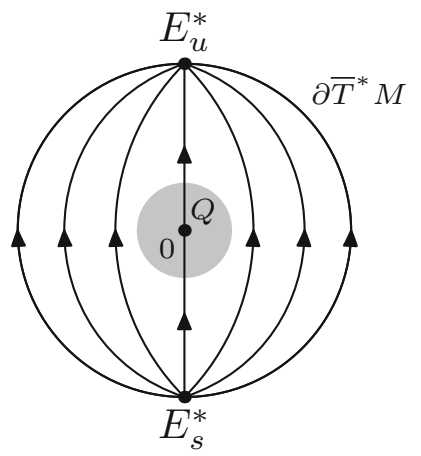

Fig. 28 A schematic representation of the flow on the compactification of $T^{*} M, \bar{T}^{*} M$-by compactification we mean replacing each fiber $T_{x}^{*} M$ by a ball $\bar{T}_{x}^{*} M$ with the boundary corresponding to the sphere at infinity. At zero energy, $p^{-1}(0)=\left\{(x, \xi): \xi\left(X_{x}\right)=0\right\}$ the trapped set of the flow is given by the zero section and the operator $X / i-i Q(x, h D) / h-\lambda$ is invertible on spaces $H_{r}$ (with uniform bounds once the norms are semiclassical modified by changing $G(x, D)$ to $G(x, h D)$ ) for $Q \geq 0$ microlocalized to the shaded region and elliptic in a neighbourhood of the trapped set and $\operatorname{Im} \lambda>-r / C_{0}$. Since $Q(x, h D)$ is a compact operator that, and estimates on the adjoint on dual spaces, shows the Fredholm property of $X / i-\lambda$

see [136, Definition 8.1.2] and [137, Theorem 18.1.27] for a useful characterization. The definition means that the Fourier transform of a localization of $u$ to a neighbourhood of $x$ decays rapidly in a conic neighbourhood of $\xi$. Rapid decay in all directions would mean that $u$ is smooth at $x$ and hence $\mathrm{WF}(u)$ provides a phase space localized description of smoothness. We also recall [136, Definition 8.2.2]: for a closed conic subset, $\Gamma$, of $T^{*} M \backslash 0$ ( 0 denotes the zero section $\xi=0$; conic means the invariance under the multiplicative action of $\mathbb{R}_{+}$on the fibers),

$$
\mathcal{D}_{\Gamma}^{\prime}(M):=\left\{u \in \mathcal{D}_{\Gamma}^{\prime}(M): \operatorname{WF}(u) \subset \Gamma\right\}
$$

With this notation we can say that there exist conic neihbourhoods, $\Gamma_{s}$ and $\Gamma_{u}$ if $E_{u}^{*}$ and $E_{s}^{*}$ respectively such that for $r \geq 0$,

$$
\begin{aligned}
& H_{r G}(M) \cap \mathcal{D}_{\Gamma_{u}}^{\prime}(M)=H^{-r}(M) \cap \mathcal{D}_{\Gamma_{u}}^{\prime}(M), \\
& H_{r G}(M) \cap \mathcal{D}_{\Gamma_{s}}^{\prime}(M)=H^{r}(M) \cap \mathcal{D}_{\Gamma_{s}}^{\prime}(M) .
\end{aligned}
$$

Hence, our spaces improve regularity along the flow (see Fig. 28) and that leads to a Fredholm property (roughly, the inclusion $H^{r} \hookrightarrow H^{-r}, r>0$, is a compact operator). Such spaces appeared in scattering theory long ago: in the work of Melrose [180] on the Poisson formula for resonances and, with an explicit use of microlocal weights (in the analytic setting via the FBI transform), in the work of Helffer-Sjöstrand [125] on a microlocal version of complex scaling.

The following theorem was first proved by Faure-Sjöstrand [84] for more specific weights $G$ and by Dyatlov-Zworski [78]. The characterization of resonant states using a wave front set condition is implicit in [84] and is stated in Dyatlov-Faure-Guillarmou [71, Lemma 5.1] and [79, Lemma 2.2]. For non-compact $M$ with hyperbolic trapped 
sets for the flow $\varphi_{t}$ a much more complicated analogue was proved by DyatlovGuillarmou [73, Theorem 2].

Theorem 15 Suppose that $H_{r G}(M)$ is defined in (4.14), $P=\frac{1}{i} X$, where $X$ is the generator of the (Anosov) flow. Define $D_{r G}(M):=\left\{u \in H_{r G}(M): P u \in H_{r G}(M)\right\}$. There exist constants, $C_{0}, C_{1}$ such that

$$
P-\lambda: D_{r G}(M) \rightarrow H_{r G}(M), \quad r>C_{0}(\operatorname{Im} \lambda)_{-}+C_{0},
$$

is a Fredholm family of operators, invertible for $\operatorname{Im} \lambda>C_{1}$. Hence, $\lambda \mapsto(P-\lambda)^{-1}$ : $H_{r G} \rightarrow H_{r G}$ is a meromorphic family of operators in $\operatorname{Im} \lambda>-r / C_{0}$ and in particular (4.13) holds for any A. In addition,

$$
\frac{1}{2 \pi i} \operatorname{tr}_{H_{r G}(M)} \oint_{\lambda}(\zeta-P)^{-1} d \zeta=\operatorname{dim}\left\{u \in \mathcal{D}_{E_{u}^{*}}^{\prime}(M): \exists \ell(P-\lambda)^{\ell} u=0\right\} .
$$

Here the integral is over a sufficiently small positively oriented circle centered at $\lambda$.

This result remains valid for operators $\mathbf{P}: \mathcal{C}^{\infty}(M, \mathcal{E}) \rightarrow \mathcal{C}^{\infty}(M, \mathcal{E})$ where $\mathcal{E}$ is any smooth complex vector bundle and $\mathbf{P}$ is a first order differential system with the principal part given by $P$. That will be important in Sect. 4.3.

Theorem 15 provides a simple definition of Pollicott-Ruelle resonances formulated in terms of the wave front set condition and action on distributions. We state it only in the scalar case:

Definition 6 Suppose $M$ is a compact manifold, $X \in \mathcal{C}^{\infty}(M ; T M)$ generates an Anosov flow in the sense of (4.4) and $E_{u}^{*}$ is defined in (4.7).

We say that $\lambda \in \mathbb{C}$ is a Pollicott-Ruelle resonance if

$$
\exists u \in \mathcal{D}_{E_{u}^{*}}^{\prime}(M)(P-\lambda) u=0, \quad P:=\frac{1}{i} X,
$$

where $\mathcal{D}_{E_{u}^{*}}^{\prime}(M)$ is defined in (4.17). The multiplicity of the resonance is defined by the right hand side of (4.20).

Our insistence of $\frac{1}{i}$ in $P$ comes from quantum mechanician's attachment to selfadjoint operators: $P$ is self-adjoint on $L^{2}(M, d m)$ if the flow admits a smooth invariant measure $d m$. In some conventions, e.g. in [71,118], resonances are given by $s=-i \lambda$.

From the physical point of view this definition should be stable when the flow is randomly perturbed, that is if we change

$$
\dot{x}(t)=-X_{x(t)}, \quad x(0)=x \longrightarrow \dot{x}_{\varepsilon}(t)=-X_{x_{\varepsilon}(t)}+\sqrt{2 \varepsilon} \dot{B}(t), \quad x_{\varepsilon}(0)=x,
$$

where where $B(t)$ is the Brownian motion corresponding a Riemannian metric $g$ on $M$-see for instance [82]. This corresponds to changing the evolution by $P=X / i$ :

$$
e^{-i t P} f(x)=f(x(t)) \longrightarrow e^{-i t P_{\varepsilon}} f(x)=\mathbb{E}\left[f\left(x_{\varepsilon}(t)\right)\right], P_{\varepsilon}:=P+i \varepsilon \Delta_{g} .
$$


The operator $P_{\varepsilon}$ is elliptic for $\varepsilon>0$ and hence has a discrete spectrum with $L^{2}$ eigenfunctions. This spectrum converges to the Pollicott-Ruelle resonances as was shown in [77]. Stability of Pollicott-Ruelle resonances for Anosov maps has been established by Blank-Keller-Liverani [23] and Liverani [169], following a very general argument of Keller-Liverani [155].

Theorem 16 Suppose that $\lambda_{0}$ is a Pollicott-Ruelle resonance of multiplicity $m$ (see Definition 6). Then there exists $r_{0}>0, \varepsilon_{0}>0$ such that for $0<\varepsilon<\varepsilon_{0}, P_{\varepsilon}$ (defined in (4.23)) has exactly $m$ eigenvalues $\left\{\lambda_{j}(\varepsilon)\right\}_{j=1}^{m}$ in $D\left(\lambda_{0}, r_{0}\right)$, and

$$
\lambda_{j}(\varepsilon) \rightarrow \lambda_{0}
$$

with the convergence uniform for $\lambda_{0}$ in a compact set.

Moreover, if $(M, \alpha)$ is a contact manifold and $X$ is its Reeb vector field (see (4.8)), then for any $\delta>0$ there exist $R>0$ such that for all $\varepsilon>0$,

$$
\operatorname{Spec}\left(P_{\varepsilon}\right) \cap\left([R, \infty)-i\left[0, \frac{1}{2}\left(\gamma_{0}-\delta\right)\right]\right)=\emptyset,
$$

where

$$
\gamma_{0}=\liminf _{t \rightarrow \infty} \frac{1}{t} \inf _{x \in M} \log \operatorname{det}\left(\left.d \gamma_{t}\right|_{E_{u}(x)}\right)
$$

The last statement means that an asymptotic resonance free region for contact flows (see Sect. 4.4) is stable under random perturbations. It also comes with polynomial resolvent bounds $\left(P_{\varepsilon}-\lambda\right)^{-1}=\left(|\lambda|^{N_{0}}\right)_{H^{s_{0}} \rightarrow H^{-s_{0}}}$. Stability of the gap for certain maps was established by Nakano-Wittsten [195].

An elliptic perturbation $P_{\varepsilon}=X / i+i \varepsilon \Delta_{g}$ is natural when there is no additional structure. However, for Hamiltonian systems, or more specifically, for Anosov geodesic flows on Riemannian manifolds $(\Sigma, g)$ — see (4.9)—a more natural operator is given by

$$
\begin{aligned}
\widetilde{P}_{\varepsilon} & :=\frac{1}{i} X+i \varepsilon \widetilde{\Delta}_{g}, \\
\widetilde{\Delta}_{g} u(z, \omega) & :=\sum_{i, j}^{n} g_{i j}(z) \partial_{\xi_{i} \xi_{j}}^{2}\left[u\left(z, \xi /|\xi|_{g}\right)\right], \\
\omega & =\xi /|\xi|_{g}, \quad u(z, \bullet) \in \mathcal{C}^{\infty}\left(S_{z}^{*} \Sigma\right) .
\end{aligned}
$$

The operator $\widetilde{P}_{\varepsilon}$ is now only hypoelliptic and the Brownian motion "kick" occurs only in the momentum variables which is physically natural. The analogue of the first part of Theorem 16 was recently proved by Drouot [62] and that paper can be consulted for background information and many references. The proof combined methods of [77] and semiclassical hypoelliptic estimates inspired by the work of Lebeau [165]. An alternative approach to these estimates was given by Smith [251] who adapted his earlier paper [250] to the semiclassical setting (Fig. 29).

We formulate the $\widetilde{P}_{\varepsilon}$ analogue of the second part of Theorem 16 as 


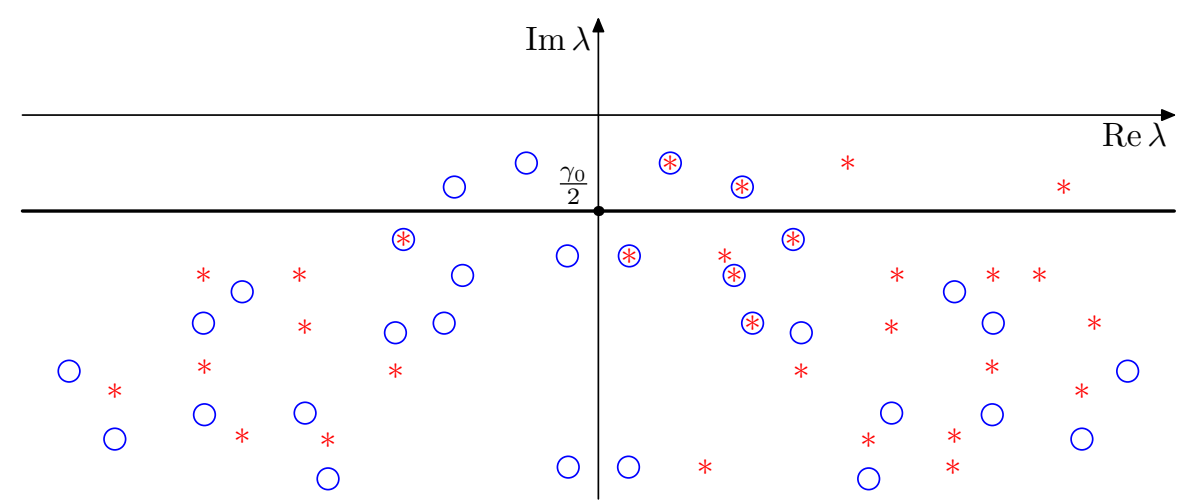

Fig. 29 A schematic presentation of the results in Theorems 16. Pollicott-Ruelle resonances of the generator of the flow $X$ (denoted by red asterisks) are approximated by the eigenvalues of $X / i+i \varepsilon \Delta_{g}$ (denoted by blue circles) uniformly on compact sets. The asymptotic resonance free strip is uniform with respect to $\varepsilon$ (colour figure online)

Conjecture 8 Suppose $M=S^{*} \Sigma$, where $(\Sigma, g)$ is a compact Riemannian manifold whose geodesic flow is an Anosov flow. Then the second part of Theorem 16 holds with $\widetilde{P}_{\varepsilon}$ in place of $P_{\varepsilon}$.

\subsection{Connections to dynamical zeta functions}

In addition to expansions of correlations, the study of Pollicott-Ruelle resonances is also motivated by their appearance as poles and zeros of dynamical zeta functions. For motivation and history of that rich subject we refer to [109, §1] and [214]. Here we will consider the Ruelle zeta function [226] which is defined by analogy with the Riemann zeta function, $\zeta(s)=\prod_{p}\left(1-p^{-s}\right)^{-1}$, with primes $p$ replaced by lengths of primitive closed geodesics

$$
\zeta_{R}(\lambda):=\prod_{\gamma \in \mathcal{G}}\left(1-e^{i \lambda \ell_{\gamma}}\right) .
$$

(We switch to our convention $\lambda=i s$.) Here $\mathcal{G}$ denotes the set of primitive closed trajectories (that is trajectories on which one "goes around only once") of $\varphi_{t}$ and $\ell_{\gamma}$ is the length of the trajectory $\gamma$.

One question, asked by Smale in [249, §II.4] was if $\zeta_{R}$ admits a meromorphic continuation to $\mathbb{C}^{12}$ or to large strips $\operatorname{Im} \lambda>-A$. When $M=S^{*} \Sigma$ (see (4.9)) where $(\Sigma, g)$ is a compact Riemannian surface of negative curvature, that had already been known thanks to the Selberg trace formula and the meromorphy of the Selberg zeta function:

$$
\zeta_{S}(s):=\prod_{\gamma \in \mathcal{G}} \prod_{m=0}^{\infty}\left(1-e^{-(m+s) \ell_{\gamma}}\right), \quad \zeta_{R}(i s)=\frac{\zeta_{S}(s)}{\zeta_{S}(s+1)},
$$

\footnotetext{
$\overline{12 \text { I cannot resist recalling that Smale referred to it as a "wild idea" }}$ and wrote "I must admit a positive answer would be a little shocking!"
} 
see for instance [173, Theorem 5] for a self-contained presentation.

The first zero of $\zeta_{R}$ is related to topological entropy (the value of the pressure at 0 in the notation of (3.29)) and the continuation to a small strip past that first zero was achieved by Parry-Pollicott [204]. To obtain larger strips turns out to be as difficult as obtaining global meromorphic continuation (which proceeds strip by strip; in the case of $C^{k}$ flows the meromorphy only holds in a strip of size depending on $k$-see [109]; our microlocal arguments give that as well but with less precision). When the manifold $M$ and the flow are real analytic that continuation was obtained by Rugh [228] and Fried [94]. For Anosov flows on smooth compact manifolds it was first established by Giulietti-Liverani-Pollicott [109] and then by Dyatlov-Zworski [78]. Dyatlov-Guillarmou [73] considered the more complicated non-compact case and essentially settled the original conjecture of Smale.

We will now explain the proof [78] of the meromorphic continuation of $\zeta_{R}$. The first step [226] is a factorization of the zeta function valid in the case when the stable and unstable bundles, $x \mapsto E_{S}(x), x \mapsto E_{u}(x)$, respectively, are orientable: ${ }^{13}$

$$
\zeta_{R}(\lambda)=\prod_{j=0}^{n-1} \zeta_{j}(\lambda)^{(-1)^{j+q}}, \quad \zeta_{j}(\lambda):=\exp \left(-\sum_{k=1}^{\infty} \sum_{\gamma \in \mathcal{G}} \frac{e^{i \lambda k \ell_{\gamma}} \operatorname{tr} \wedge^{j} \mathcal{P}_{\gamma}^{k}}{k\left|\operatorname{det}\left(I-\mathcal{P}_{\gamma}^{k}\right)\right|}\right)
$$

where $\operatorname{dim} M=n, q=\operatorname{dim} E_{s}$ and the Poincaré map is defined by

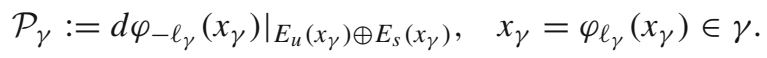

Since we are taking determinants and traces, $\zeta_{j}$ 's do not depend on the choices of $x_{\gamma}$. The factorization essentially follows from $\operatorname{det}(I-A)=\sum_{j=0}^{n-1}(-1)^{j} \operatorname{tr} \wedge^{j} A-$ see $[78, \S 2.2]$.

The relation of the zeta functions $\zeta_{j}$ with the flow by the Atiyah-Bott-Guillemin trace formula (see [78, Appendix B] for a proof):

$$
\begin{aligned}
\operatorname{tr}^{\mathrm{b}} e^{-i t \mathbf{P}_{j}} & =\sum_{k=1}^{\infty} \sum_{\gamma \in \mathcal{G}} \frac{\ell_{\gamma} \operatorname{tr} \wedge^{j} \mathcal{P}_{\gamma}^{k} \delta\left(t-k \ell_{\gamma}\right)}{\left|\operatorname{det}\left(I-\mathcal{P}_{\gamma}^{k}\right)\right|}, t>0, \\
\mathbf{P}_{j} & =\frac{1}{i} \mathcal{L}_{X}: \mathcal{C}^{\infty}\left(M ; \mathcal{E}_{0}^{j}\right) \rightarrow \mathcal{C}^{\infty}\left(M ; \mathcal{E}_{0}^{j}\right),
\end{aligned}
$$

where $\mathcal{E}_{0}^{j}$ be the smooth invariant verctor bundle of all differential $j$-forms $\mathbf{u}$ satisfying $\iota_{V} \mathbf{u}=0$, where $\iota$ denotes the contraction operator by a vector field.

The flat trace, $\operatorname{tr}^{b}$, is defined using operations on distributions which generalize integration of the Schwartz kernel over the diagonal: let $J: \mathbb{R}_{+} \times M \rightarrow \mathbb{R}_{+} \times M \times M$ be given by $J(t, x)=(t, x, x)$ and $\pi: \mathbb{R}_{+} \times M \rightarrow \mathbb{R}_{+}$, by $\pi(t, x)=t$. If $K \in$ $\mathcal{D}^{\prime}\left(\mathbb{R}_{+} \times M \times M\right)$ is the Schwartz kernel of the operator $e^{-i t P}=\varphi_{-t}^{*}$ then

$$
\operatorname{tr}^{b} e^{-i t \mathbf{P}_{j}}:=\pi_{*} J^{*} K \in \mathcal{D}^{\prime}\left(\mathbb{R}_{+}\right)
$$

13 See [109, Appendix B] for the modifications needed in the non-orientable case. 
The push forward of a distribution $\pi_{*}$ is always well defined in case compact manifolds but that is not the case for a pullback by an inclusion $J^{*}$ which, if $K$ were smooth would be

$$
J^{*} K(t, x):=K(t, x, x)
$$

- see [136, Theorem 8.2.4, Corollary 8.2.7]. The condition which justifies (4.33) for distributional $K$ is

$$
\begin{aligned}
\mathrm{WF}(K) \cap N^{*}\left(\mathbb{R}_{+} \times \Delta\right) & =\emptyset, \\
\mathbb{R}_{+} \times \Delta & :=\left\{(t, x, x):(t, x) \in \mathbb{R}_{+} \times \Delta\right\},
\end{aligned}
$$

where the wave front set WF was defined in (4.16) and $N^{*}\left(\mathbb{R}_{+} \times \Delta\right) \subset T^{*}\left(\mathbb{R}_{+} \times M \times\right.$ $M) \backslash 0$ is the conormal bundle of $\mathbb{R}_{+} \times \Delta$, that is, the annihilator of $T\left(\mathbb{R}_{+} \times \Delta\right) \subset$ $T\left(\mathbb{R}_{+} \times M \times M\right)$.

The condition (4.34) is satisfied for $K$ given by the Schwartz kernel of $e^{-i t P}=\varphi_{-t}^{*}$, $t>0$, because for Anosov flows powers of the Poincaré map (4.30), $\mathcal{P}_{\gamma}^{k}$, cannot have 1 as an eigenvalue. Hence (4.32) makes sense and (4.31) holds.

We now observe that for $\operatorname{Im} \lambda \gg 1$ and for $0<t_{0}<\min _{\mathcal{G}} \ell_{\gamma}$,

$$
\frac{1}{i} \int_{t_{0}}^{\infty} \operatorname{tr}^{\mathrm{b}} e^{-i t\left(\mathbf{P}_{j}-\lambda\right)} d t=\zeta_{j}(\lambda)^{-1} \frac{d}{d \lambda} \zeta_{j}(\lambda) .
$$

In view of (4.29), to show that $\zeta_{R}$ has a meromorphic extension, it is enough to show that each $\zeta_{j}$ has a holomorphic extension. That in turn follows from a meromorphic extension of $\zeta_{j}^{\prime} / \zeta_{j}$ with simple poles and integral residues.

Hence we need to show that the left hand side of (4.35) has a meromorphic extension to $\mathbb{C}$ with poles of finite rank. This is clearly related to (4.13). A formal manipulation suggests that the left hand side of (4.35) is given by

$$
-e^{i t_{0} \lambda} \operatorname{tr}^{\mathrm{b}}\left(\varphi_{-t_{0}}^{*}\left(\mathbf{P}_{j}-\lambda\right)^{-1}\right)=-e^{i t_{0} \lambda}\left\langle J^{*}\left(\varphi_{-t_{0}} \otimes \mathrm{id}\right)^{*} \mathbf{K}_{j}(\lambda), 1\right\rangle
$$

where $\mathbf{K}_{j}(\lambda)$ is the Schwartz kernel of $\left(\mathbf{P}_{j}-\lambda\right)^{-1}$ (which makes sense for all $\lambda$ in view of Theorem 15), $J(x)=(x, x)$ and $\langle\bullet, \bullet\rangle$ is the distributional pairing $\mathcal{D}^{\prime}(M) \times \mathcal{C}^{\infty}(M) \rightarrow \mathbb{C}$. Just as in (4.34) this is justified by showing that for $0<t_{0}<\min _{\gamma \in \mathcal{G}} \ell_{\gamma}$,

$$
\mathrm{WF}\left(\left(\varphi_{-t_{0}} \otimes \mathrm{id}\right)^{*} \mathbf{K}_{j}(\lambda)\right) \cap N^{*} \Delta=\emptyset, \quad \Delta:=\{(x, x): x \in M\} .
$$

We used the notation $\left(\varphi_{-t_{0}} \otimes \mathrm{id}\right)(x, y):=\left(\varphi_{-t_{0}}(x), y\right)$ and the pullback by that diffeomorphism of $M \times M$ is always well defined [136, Theorem 8.2.4]. We have to be careful at the poles of $\mathbf{K}_{j}(\lambda)$ but a decomposition similar to (2.23) shows that we can separate a holomorphic and singular parts. On the singular part $\operatorname{tr}^{\mathrm{b}}=\operatorname{tr}_{H_{r G}}$ which kills all the higher order poles (nilpotency of Jordan blocks) and gives an integral residue. 
Condition (4.37) is an immediate consequence of [78, Proposition 3.3] (though it takes a moment to verify it: the pullback by $\left(\varphi_{-t_{0}} \otimes \mathrm{id}\right)$ shifts things away from the diagonal):

Theorem 17 Let $\mathbf{K}_{j}(\lambda) \in \mathcal{D}^{\prime}(M)$ be the Schwartz kernel of $\left(\mathbf{P}_{j}-\lambda\right)^{-1}$ : $\mathcal{C}^{\infty}\left(M, \mathcal{E}_{0}^{j}\right) \rightarrow \mathcal{D}^{\prime}\left(M, \mathcal{E}_{0}^{j}\right)$. Then away from the poles of $\lambda \mapsto \mathbf{K}_{j}(\lambda)$,

$$
(x, y ; \xi,-\eta) \in \mathrm{WF}\left(\mathbf{K}_{j}(\lambda)\right) \Longrightarrow(x, \xi, y, \eta) \in \Delta\left(T^{*} M\right) \cup \Omega_{+} \cup\left(E_{u}^{*} \times E_{s}^{*}\right),
$$

where $\Delta\left(T^{*} M\right):=\left\{(\rho, \rho): \rho \in T^{*} M\right\} \subset T^{*} M \times T^{*} M$ and

$$
\Omega_{+}:=\left\{\left(e^{t H_{p}}(x, \xi), x, \xi\right): t \geq 0, p(x, \xi)=0\right\}, p(x, \xi):=\xi\left(X_{x}\right) .
$$

Away from the conic sets $E_{u}^{*} \times E_{s}^{*}$ this follows from a modification of results of Duistermaat-Hörmander on propagation of singulaties [78, Proposition 2.5]. Near $E_{s}^{*}$ we use a modification of Melrose's propagation result [183] for radial sources [78, Proposition 2.6], and near $E_{u}^{*}$, his propagation result for radial sinks [78, Proposition 2.7]. The property (4.18) is crucial here and implies the relation between $r$ and $\operatorname{Im} \lambda$ in Theorem 15 . Except for the fact that $E_{u}^{*}$ and $E_{s}^{*}$ are typically very irregular as sets, these are the same estimates that Vasy [263] used to prove Theorem 8 presented Sect. 3.1.

Retracing our steps through (4.37), (4.36), (4.35) and (4.29) we see that we proved the theorem of Giulietti-Liverani-Pollicott [109] settling Smale's conjecture [249, $\S I I .4]$ in the case of compact manifolds. For the full conjecture in the non-compact case proved by a highly nontrivial elaboration of the above strategy, see DyatlovGuillarmou [73]:

Theorem 18 The Ruelle zeta function, $\zeta_{R}(\lambda)$, defined for $\operatorname{Im} \lambda \gg 1$ by (4.27), continues meromorphically to $\mathbb{C}$.

What the method does not recover is the order of $\zeta_{R}(\lambda)$ in the case when $M$ and $X$ are real analytic [94,228]. That may be related to issues around Conjecture 2.

Pollicott-Ruelle resonances of $\mathbf{P}_{j}$ 's are exactly the zeros of the entire functions $\zeta_{j}(\lambda)$ 's. The simple Definition 6 using wave front set characterization led to a short proof of the following fact [79]: suppose that $(\Sigma, g)$ is an oriented negatively curved Riemannian surface. Then

$$
\zeta_{R}(\lambda)=c \lambda^{|\chi(\Sigma)|}(1+\mathcal{O}(\lambda)), \quad c \neq 0, \quad \lambda \rightarrow 0,
$$

where $\chi(\Sigma)$ is the Euler characteristic of $\Sigma$. In particular this implies that lengths of closed geodesics determine the genus of the surface linking dynamics and topology. Previous to [79] that was known only for surfaces of constant curvature.

Hence (4.39) provides evidence that results valid in rigid (that is, locally symmetric) geometries may be valid in greater generality. Thus, consider a compact oriented 
negatively curved Riemannian manifold $(\Sigma, g)$. Following Fried [92,94], for a representation $\alpha: \pi_{1}\left(S^{*} \Sigma\right) \rightarrow G L(N, \mathbb{C})$, we define

$$
\zeta_{\alpha}(\lambda):=\prod_{\gamma \in \mathcal{G}} \operatorname{det}\left(I-\alpha(\gamma) e^{i \lambda \ell_{\gamma}}\right) .
$$

The proof of meromorphy of $\zeta_{R}$ given in [78] and sketched above gives also the meromorphic continuation of $\zeta_{\alpha}$ : we only need to change the vector bundles on whose sections $\frac{1}{i} \mathcal{L}_{X}$ acts. The following statement was proved by Fried [92] in the case of manifolds of constant negative curvature and conjectured (in an even more precise form) in [94, p.181] for more general manifolds. We refer to his papers [92,94] for precise definitions of the objects involved in the statement:

Conjecture 9 Suppose that $(\Sigma, g)$ is a negatively curved oriented compact Riemannian manifold of dimension $n>2$ and that $\alpha$ is an acyclic unitary representation of $\pi_{1}(\Sigma)$. Then

$$
\left|\zeta_{\alpha}(0)\right|^{(-1)^{n-1}}=\left|T_{\alpha}(\Sigma)\right|^{2}
$$

where $\zeta_{\alpha}$ is defined by (4.40) and $T_{\alpha}(\Sigma)$ is the analytic torsion of $\Sigma$.

The analytic torsion $T_{\alpha}(\Sigma)$ was defined by Ray and Singer using eigenvalues of an $\alpha$-twisted Hodge Laplacian. Their conjecture that $T_{\alpha}(\Sigma)$ is equal to the Reidemeister torsion, a topological invariant, was proved independently by Cheeger and Müller. Hence (4.41) would link dynamical, spectral and topological quantities. In the case of locally symmetric manifolds a more precise version of the conjecture was recently proved by Shen [237, Theorem 4.1] following earlier contributions by Bismut [22] and Moskovici-Stanton [190].

\subsection{Distribution of Pollicott-Ruelle resonances}

Exponential decay of correlations (4.3) was established by Dolgopyat [59] for systems which include geodesic flows on negatively curved compact surfaces and by Liverani [168] for all contact Anosov flows, hence in particular for geodesics flows on negatively curved compact manifolds-see (4.8), (4.9). These two papers can also be consulted for the history of the subject. See also Baladi-Demers-Liverani [7] for some very recent progress in the case of hyperbolic billiards.

Comparing (4.3) and (4.12) shows that if we know a resonance free strip with a polynomial bound on $(P-\lambda)^{-1}$ in that strip then we obtain exponential decay of correlations. Hence we define the following two spectral (though really "scattering") quantities: the spectral gap (for simplicity we consider only the scalar case):

$$
v_{1}=\sup \{v: \operatorname{Res}(P) \cap\{\operatorname{Im} \lambda>-v\}=\{0\}\},
$$

and the essential spectral gap:

$$
v_{0}=\sup \{v:|\operatorname{Res}(P) \cap\{\operatorname{Im} \lambda>-v\}|<\infty\} .
$$


Here $\operatorname{Res}(P)$ denotes the set of resonances of $P$. Obviously if $\nu_{0}>0$ and if we know that there are no non-zero real resonances, then $v_{1}>0$.

We have the following result which implies exponential decay of correlations for contact Anosov flows:

Theorem 19 Suppose that $(M, \alpha)$ is a compact contact manifold and that the Reeb vector field (4.8) generates an Anosov flow. Then in the notation of (4.43) and (4.25),

$$
\frac{1}{2} \gamma_{0} \leq v_{0}<\infty
$$

Moreover, for some s

$$
\begin{gathered}
\forall \delta>0 \exists C, N \quad \begin{array}{c}
\left\|(P-\lambda)^{-1}\right\|_{H^{s} \rightarrow H^{-s}} \leq C(1+|\lambda|)^{N} \\
\text { for }|\operatorname{Re} \lambda|>C, \operatorname{Im} \lambda>-v_{0}+\delta
\end{array}
\end{gathered}
$$

One could estimate $s$ more precisely depending on the width of the strip which would give better decay of correlations results.

The lower bound in (4.44) was proved by Tsujii [261] who improved the result of Liverani at high energies. It also follows from the very general results of [202] which apply also in quantum scattering. The method of [202] is crucial in obtaining the second part of Theorem 16-see also Conjecture 8.

The finiteness of $v_{0}$ follows from a stronger statement in Jin-Zworski [153]: for any Anosov flow there exist strips with infinitely many Pollicott-Ruelle resonances.

One immediate consequence of Theorem 19 is the analogue of Theorems 3 and 12 in the setting of Anosov flows: suppose we enumerate the non-zero resonances so that $\operatorname{Im} \lambda_{j+1} \leq \operatorname{Im} \lambda_{j}<0^{14}$. There exist distributions $u_{j, k}, v_{j, k} \in \mathscr{D}^{\prime}(M), 0 \leq k \leq K_{j}$, such that, for any $\delta>0$, there exists $J(\delta) \in \mathbb{N}$ such that for any $f, g \in \mathcal{C}^{\infty}(M)$

$\rho_{f, g}(t)=\int_{M} f d m \int_{M} g d m+\sum_{j=1}^{J(\delta)} \sum_{k=1}^{K_{j}} t^{k} e^{-i t \mu_{j}}\left\langle u_{j, k}, f\right\rangle\left\langle v_{j, k}, g\right\rangle+\mathcal{O}_{f, g}\left(e^{-t\left(\nu_{0}-\delta\right)}\right)$,

for $t>0$. Here $d m$ is the invariant density coming from the contact form and normalized to have $\int_{M} d m=1$ and $\langle\bullet, \bullet\rangle$ denotes distributional pairing. This refines (4.3) and provides a rigorous version of (4.2).

We conclude with a review of counting results for resonances. We put

$$
N(\Omega):=|\operatorname{Res}(P) \cap \Omega|, \quad \Omega \Subset \mathbb{C},
$$

and denote $n:=\operatorname{dim} M$.

\footnotetext{
14 There are no non-zero real resonances for Anosov flows with smooth invariant measures and in particular for contact Anosov flows-for a direct microlocal argument for that see [79, Lemma 2.3].
} 
The first bound was proved by Faure-Sjöstrand [84] and it holds for general Anosov flows on compact manifolds: for any $\gamma>0$,

$$
N\left(\left[r-r^{\frac{1}{2}}, r+r^{\frac{1}{2}}\right]-i[0, \gamma]\right)=o\left(r^{n-\frac{1}{2}}\right) .
$$

For Anosov contact flows a sharp bound was proved by Datchev-Dyatlov-Zworski and it says that

$$
N([r-1, r+1]-i[0, \gamma])=\mathcal{O}\left(r^{\frac{n-1}{2}}\right),
$$

which improves (4.47), giving $N\left(\left[r-r^{\frac{1}{2}}, r+r^{\frac{1}{2}}\right]-i[0, \gamma]\right)=O\left(r^{\frac{n}{2}}\right)$, in the contact case. These bounds are sharp in all dimensions as shown by the very precise analysis of Pollicott-Ruelle resonances on compact hyperbolic quotients by Dyatlov-FaureGuillarmou [71]. Although these resonances have been known for a long time in the case of surfaces, in higher dimensions some new structure was discovered in [71], see also Guillarmou-Hilgert-Weich [118].

Under a pinching condition on minimal and maximal expansion rates $v_{\max }<2 v_{\min }$, Faure-Tsujii [87] proved a sharp lower bound in the contact case: for any $\varepsilon>0$ and any $\delta>0$ there exists $c>0$ such that

$$
N\left(\left[r-r^{\varepsilon}, r+r^{\varepsilon}\right]-i \frac{1}{2}\left[0, v_{\max }+\delta\right]\right) \geq c r^{\frac{n-1}{2}+\varepsilon} .
$$

This should be compared to Dyatlov's Weyl asymptotics shown in Fig. 22-see [67, $\S 1]$. The only, and very weak, bound in the general Anosov case follows from a local trace formula [153, Theorem 1] (similar in spirit to Sjöstrand's local trace formula for resonances [241]): for every $0<\delta<1$ there exists $A=A_{\delta}$ such that in the Hardy-Littlewood notation, ${ }^{15}$

$$
N([0, r]-i[0, A])=\Omega\left(r^{\delta}\right) .
$$

However, Naud observed [153, Appendix B] that for suspensions of certain Anosov maps, the bound $\mathcal{O}(r)$ holds, in which case (4.50) is not too far off.

This state of affairs shows that many problems remain and the non-compact case seems to be completely open.

Acknowledgements I would like thank my collaborator on [80], Semyon Dyatlov, for allowing me to use some of the expository material from our book, including figures, in the preparation of this survey and for his constructive criticism of earlier versions. Many thanks go also to David Borthwick, Alexis Drouot, Jeff Galkowski, Peter Hintz, Jian Wang and Steve Zelditch for their comments and corrections. I am also grateful to Hari Manoharan, Ulrich Kuhl, Plamen Stefanov, David Bindel, Mickael Chekroun, Eric Heller and David Borthwick for allowing me to use Figs. 1, 2, 8, 11, 13, 14, 19, 21 and 26, respectively. The work on this survey was supported in part by the National Science Foundation grant DMS-1500852.

Open Access This article is distributed under the terms of the Creative Commons Attribution 4.0 International License (http://creativecommons.org/licenses/by/4.0/), which permits unrestricted use, distribution, and reproduction in any medium, provided you give appropriate credit to the original author(s) and the source, provide a link to the Creative Commons license, and indicate if changes were made.

$15 \Omega(f)=g$ if it is not true that $f=o(|g|)$. 


\section{References}

1. Abbott, B.P., et al.: (LIGO Scientific Collaboration and Virgo Collaboration), Observation of gravitational waves from a binary black hole merger. Phys. Rev. Lett. 116, 061102 (2016)

2. Agmon, S.: Spectral theory of Schrödinger operators on Euclidean and non-Euclidean spaces. Commun. Pure Appl. Math. 39(Number S, Supplement) (1986)

3. Aguilar, J., Combes, J.M.: A class of analytic perturbations for one-body Schrödinger Hamiltonians. Commun. Math. Phys. 22, 269-279 (1971)

4. Alexandrova, I., Tamura, H.: Resonances in scattering by two magnetic fields at large separation and a complex scaling method. Adv. Math. 256, 398-448 (2014)

5. Baladi, V.: Positive Transfer Operators and Decay of Correlations, Advanced Series in Nonlinear Dynamics, vol. 16. World Scientific Publishing Co., Inc, River Edge (2000)

6. Baladi, V.: The quest for the ultimate anisotropic Banach space. arXiv: 1607.00654

7. Baladi, V., Demers, M., Liverani, C.: Exponential Decay of correlations for finite horizon Sinai billiard flows. arXiv: 1506.02836

8. Baladi, V., Eckmann, J.-P., Ruelle, D.: Resonances for intermittent systems. Nonlinearity 2, 119-131 (1989)

9. Baladi, V., Tsujii, M.: Anisotropic Hölder and Sobolev spaces for hyperbolic diffeomorphisms. Ann. Inst. Fourier 57, 127-154 (2007)

10. Balslev, E., Combes, J.M.: Spectral properties of many-body Schrödinger operators wth dilation analytic interactions. Commun. Math. Phys. 22, 280-294 (1971)

11. Baouendi, M.S., Goulaouic, C.: Cauchy problems with characteristic initial hypersurface. Commun. Pure Appl. Math. 26, 455-475 (1973)

12. Bardos, C., Lebeau, G., Rauch, J.: Scattering frequencies and Gevrey 3 singularities. Invent. Math. 90, 77-114 (1987)

13. Barkhofen, S., Weich, T., Potzuweit, A., Kuhl, U., Stöckmann, H.-J., Zworski, M.: Experimental observation of spectral gap in microwave $n$-disk systems. Phys. Rev. Lett. 110, 164102 (2013)

14. Baskin, D., Spence, E., Wunsch, J.: Sharp high-frequency estimates for the Helmholtz equation and applications to boundary integral equations. SIAM J. Math. Anal. 48, 229-267 (2016)

15. Baskin, D., Vasy, A., Wunsch, J.: Asymptotics of radiation fields in asymptotically Minkowski space. Am. J. Math. 137, 1293-1364 (2015)

16. Baskin, D., Wunsch, J.: Resolvent estimates and local decay of waves on conic manifolds. J. Differ. Geom. 95, 183-214 (2013)

17. Barr, M.C., Zaletel, M.P., Heller, E.J.: Quantum corral resonance widths: lossy scattering as acoustics. Nano Lett. 10, 3253-3260 (2010)

18. Bledsoe, M., Weikard, R.: The inverse resonance problem for left-definite Sturm-Liouville operators. J. Math. Anal. Appl. 423, 1753-1773 (2015)

19. Berenger, J.P.: A perfectly matched layer for the absorption of electromagnetic waves. J. Comput. Phys. 114, 185-200 (1994)

20. Bindel, D., Govindjee, S.: Elastic PMLs for resonator anchor loss simulations. Int. J. Numer. Methods Eng. 64, 789-818 (2005)

21. Bindel, D., Zworski, M.: Theory and computation of resonances in 1d scattering. http://www.cs. cornell.edu/\%7Ebindel/cims/resonant1d/

22. Bismut, J.-M.: Hypoelliptic Laplacian and orbital integrals. Annals of Mathematics Studies 177. Princeton University Press, Princeton (2011)

23. Blank, M., Keller, G., Liverani, C.: Ruelle-Perron-Frobenius spectrum for Anosov maps. Nonlinearity 15, 1905-1973 (2002)

24. Bolley, P., Camus, J.: Sur une classe d'opérateurs elliptiques et dégénérés à plusieurs variables. Mémoire Soc. Math. France 34, 55-140 (1973)

25. Bolley, P., Camus, J., Hanouzet, B.: Étude de l'analyticité et de la régularité Gevrey pour une classe de problèmes aux limites elliptiques et dégénérés. Asterisque 19, 25-48 (1974)

26. Bony, J.-F., Bruneau, V., Raikov, G.: Counting function of characteristic values and magnetic resonances. Commun. Partial Differ. Equ. 39, 274-305 (2014)

27. Bony, J.-F., Burq, N., Ramond, T.: Minoration de la résolvante dans le cas captif. C. R. Acad. Sci. Paris Sér. I Math 348(23-24), 1279-1282 (2010)

28. Bony, J.-F., Fujiie, S., Ramond, T., Zerzeri, M.: Resonances for homoclinic trapped sets. arXiv: 1603.07517 
29. Bony, J.-F., Häfner, D.: Decay and non-decay of the local energy for the wave equation on the de Sitter-Schwarzschild metric. Commun. Math. Phys. 282, 697-719 (2008)

30. Borthwick, D.: Distribution of resonances for hyperbolic surfaces. Exp. Math. 23, 25-45 (2014)

31. Borthwick, D.: Spectral Theory of Infinite-Area Hyperbolic Surfaces, 2nd edn. Birkhäuser, Basel (2016)

32. Borthwick, D., Guillarmou, C.: Upper bounds on the number of resonances on geometrically finite hyberbolic manifolds. J. EMS 18, 997-1041 (2016)

33. Borthwick, D., Weich, T.: Symmetry reduction of holomorphic iterated function schemes and factorization of Selberg zeta functions. J. Spectr. Theory 6, 267-329 (2016)

34. Bourgain, J., Dyatlov, S.: Spectral gaps without the pressure condition

35. Bourgain, J., Gamburd, A., Sarnak, P.: Generalization of Selberg's 3/16 theorem and affine sieve. Acta Math. 207, 255-290 (2011)

36. Brown, B.M., Knowles, I., Weikard, R.: On the inverse resonance problem. J. Lond. Math. Soc. 68(2), 383-401 (2003)

37. Burq, N.: Décroissance de l'énergie locale de l'équation des ondes pour le problème extérieur et absence de résonance au voisinage du réel. Acta Math. 180(1), 1-29 (1998)

38. Burq, N.: Lower bounds for shape resonances widths of long range Schrödinger operators. Am. J. Math. 124(4), 677-735 (2002)

39. Burq, N., Guillarmou, C., Hassell, A.: Strichartz estimates without loss on manifolds with hyperbolic trapped geodesics. Geom. Funct. Anal. 20, 627-656 (2010)

40. Burq, N., Zworski, M.: Resonance expansions in semi-classical propagation. Commun. Math. Phys. 232, 1-12 (2001)

41. Butterley, O., Liverani, C.: Smooth Anosov flows: correlation spectra and stability. J. Mod. Dyn. 1, 301-322 (2007)

42. Cao, H., Wiersig, J.: Dielectric microcavities: model systems for wave chaos and non-Hermitian physics. Rev. Mod. Phys. 87, 61-111 (2015)

43. Cardoso, F., Vodev, G.: Uniform estimates of the resolvent of the Laplace-Beltrami operator on infinite volume Riemannian manifolds II. Ann. Henri Poincaré 3(4), 673-691 (2002)

44. Chekroun, M., Neelin, J.D., Kondrashov, D., McWilliams, J.C., Ghil, M.: Rough parameter dependence in climate models and the role of Pollicott-Ruelle resonances. Proc. Natl. Acad. Sci. 111(5), 1684-1690 (2014)

45. Christiansen, T.: Some lower bounds on the number of resonances in Euclidean scattering. Math. Res. Lett. 6, 203-211 (1999)

46. Christiansen, T.: Schrödinger operators with complex-valued potentials and no resonances. Duke Math. J. 133, 313-323 (2006)

47. Christiansen, T., Hislop, P.: The resonance counting function for Schrödinger operators with generic potentials. Math. Res. Lett. 12, 821-826 (2005)

48. Christiansen, T.: Lower bounds for resonance counting functions for obstacle scattering in even dimensions. arXiv: 1510.04952

49. Dang, N.V., Riviére, G.: Spectral analyss of Morse-Smale gradient flows. arXiv: 1605.05516

50. Datchev, K.: Local smoothing for scattering manifolds with hyperbolic trapped sets. Commun. Math. Phys. 286(3), 837-850 (2009)

51. Datchev, K.: Quantitative limiting absorption principle in the semiclassical limit. Geom. Funct. Anal. 24, 740-747 (2014)

52. Datchev, K.: Resonance free regions for nontrapping manifolds with cusps. Anal. PDE 9, 907-953 (2016)

53. Datchev, K., Dyatlov, S.: Fractal Weyl laws for asymptotically hyperbolic manifolds. Geom. Funct. Anal. 23, 1145-1206 (2013)

54. Datchev, K., Dyatlov, S., Zworski, M.: Resonances and lower resolvent bounds. J. Spectr. Theory 5, 599-615 (2015)

55. Datchev, K., Hezari, H.: Resonant uniqueness of radial semiclassical Schrödinger operators. AMRX 2012, 105-113 (2012)

56. Datchev, K., Kang, D., Kessler, A.: Nontrapping surfaces of revolution with long living resonances. Math. Res. Lett. 22, 23-42 (2015)

57. Datchev, K., Vasy, A.: Gluing semiclassical resolvent estimates via propagation of singularities. IMRN 23, 5409-5443 (2012) 
58. Dinh, T.-C., Vu, D.-V.: Asymptotic number of scattering resonances for generic Schrödingier operators. Commun. Math. Phys. 326, 185-208 (2014)

59. Dolgopyat, D.: On decay of correlations in Anosov flows. Ann. Math. 147, 357-390 (1998)

60. Drouot, A.: A quantitative version of Hawking radiation, arXiv: 1510.02398, to appear in Ann. Inst. Henri Poincaré

61. Drouot, A.: Scattering resonances for highly oscillatory potentials, arXiv: 1509.04198

62. Drouot, A.: Pollicott-Ruelle resonances via kinetic Brownian motion, arXiv: 1607.03841

63. Duchêne, V., Vukićević, I., Weinstein, M.: Scattering and localization properties of highly oscillatory potentials. Commun. Pure Appl. Math. 67, 83-128 (2014)

64. Duyckaerts, T., Grigis, A., Martinez, A.: Resonance widths for general Helmholtz Resonators with straight neck, arXiv: 1504.05425

65. Dyatlov, S.: Exponential energy decay for Kerr-de Sitter black holes beyond event horizons. Math. Res. Lett. 18, 1023-1035 (2011)

66. Dyatlov, S.: Asymptotic distribution of quasi-normal modes for Kerr-de Sitter black holes. Ann. Inst. Henri Poincaré 13, 1101-1166 (2012)

67. Dyatlov, S.: Resonance projectors and asymptotics for r-normally hyperbolic trapped sets. J. AMS 28, 311-381 (2015)

68. Dyatlov, S.: Asymptotics of linear waves and resonances with applications to black holes. Commun. Math. Phys. 335, 1445-1485 (2015)

69. Dyatlov, S.: Spectral gaps for normally hyperbolic trapping. Ann. Inst. Fourier 66, 55-82 (2016)

70. Dyatlov, S.: Improved fractal Weyl bounds for hyperbolic manifolds, with an appendix by Borthwick, D., Dyatlov, S., Weich, T., arXiv: 1608.02238

71. Dyatlov, S., Faure, F., Guillarmou, C.: Power spectrum of the geodesic flow on hyperbolic manifolds. Anal. PDE 8, 923-1000 (2015)

72. Dyatlov, S., Ghosh, S.: Symmetry of bound and antibound states in the semiclassical limit for a general class of potentials. Proc. AMS 138, 3203-3210 (2010)

73. Dyatlov, S., Guillarmou, C.: Pollicott-Ruelle resonances for open systems, Ann. Inst. Henri Poincaré (A), published online, arXiv: 1410.5516

74. Dyatlov, S., Jin, L.: Resonances for open quantum maps and the fractal uncertainty principle, arXiv: 1608.02238

75. Dyatlov, S., Zahl, J.: Spectral gaps, additive energy, and a fractal uncertainty principle, Geom. Funct. Anal., published online, arXiv: 1504.06589

76. Dyatlov, S., Zworski, M.: Trapping of waves and null geodesics for rotating black holes. Phys. Rev. D 88, 084037 (2013)

77. Dyatlov, S., Zworski, M.: Stochastic stability of Pollicott-Ruelle resonances. Nonlinearity 28, 35113534 (2015)

78. Dyatlov, S., Zworski, M.: Dynamical zeta functions for Anosov flows via microlocal analysis. Ann. Sci. Ec. Norm. Supér. 49, 543-577 (2016)

79. Dyatlov, S., Zworski, M.: Ruelle zeta function at zero for surfaces, arXiv: 1606.04560

80. Dyatlov, S., Zworski, M.: Mathematical theory of scattering resonances, book in preparation; http:// math.mit.edu/ dyatlov/res/

81. Eckmann, J.-P.: Resonances in dynamical systems, IXth International Congress on Mathematical Physics (Swansea, 1988), 192-207, Hilger, Bristol, 1989. http://www.uvm.edu/ pdodds/files/papers/ others/everything/eckmann1989a.pdf

82. Elworthy, K.D.: Stochastic Differential Equations on Manifolds. Cambridge University Press, Cambridge (1982)

83. Ermann, L., Frahm, K.M., Shepelyansky, D.L.: Google matrix analysis of directed networks. Rev. Mod. Phys. 87, 1261-1310 (2015)

84. Faure, F., Sjöstrand, J.: Upper bound on the density of Ruelle resonances for Anosov flows. Commun. Math. Phys. 308, 325-364 (2011)

85. Faure, F., Roy, N.: Ruelle-Pollicott resonances for real analytic hyperbolic maps. Nonlinearity 19, 1233-1252 (2006)

86. Faure, F., Roy, N., Sjöstrand, J.: Semi-classical approach for Anosov diffeomorphisms and Ruelle resonances. Open Math. J. 1, 35-81 (2008)

87. Faure, F., Tsujii, M.: Band structure of the Ruelle spectrum of contact Anosov flows. C. R. Acad. Sci. Paris Sér. I Math 351, 385-391 (2013) 
88. Faure, F., Tsujii, M.: The semiclassical zeta function for geodesic flows on negatively curved manifolds. arXiv: 1311.4932

89. Faure, F., Tsujii, M.: Semiclassical approach for the Ruelle-Pollicott spectrum of hyperbolic dynamics. In: Analytic and Probabilistic Approaches to Dynamics in Negative Curvature

90. Fay, J.D.: Fourier coefficients of the resolvent for a Fuchsian group. J. Reine Angew. Math. 293-294, 143-203 (1977)

91. Fernández, C., Lavine, R.: Lower bounds for resonance widths in potential and obstacle scattering. Commun. Math. Phys. 128, 263-284 (1990)

92. Fried, D.: Analytic torsion and closed geodesics on hyperbolic manifolds. Invent. Math. 84(3), 523540 (1986)

93. Fried, D.: Fuchsian groups and Reidemeister torsion. In: The Selberg Trace Formula and Related Topics (Brunswick, Maine, 1984), Contemp. Math. 53, 141-163, Am. Math. Soc., Providence, RI (1986)

94. Fried, D.: Meromorphic zeta functions for analytic flows. Commun. Math. Phys. 174, 161-190 (1995)

95. Fujiié, S., Lahmar-Benbernou, A., Martinez, A.: Width of shape resonances for non globally analytic potentials. J. Math. Soc. Jpn. 63, 1-78 (2011)

96. Galkowski, J.: Distribution of resonances in scattering by thin barriers. arXiv: 1404.3709

97. Galkowski, J.: The quantum sabine law for resonances in transmission problems. arXiv: 1511.05091

98. Galkowski, J.: Resonances for thin barriers on the circle. J. Phys. A 49, 125205 (2016)

99. Galkowski, J.: A quantitative vainberg method for black box scattering. Commun. Math. Phys. (2016) arXiv: 1511.05894

100. Galkowski, J., Smith, H.: Restriction bounds for the free resolvent and resonances in lossy scattering. International Mathematics Research Notices, 7473-7509 (2015)

101. Gannot, O.: A global definition of quasinormal modes for Kerr-AdS Black Holes. arXiv: 1407.6686

102. Gannot, O.: From quasimodes to resonances: exponentially decaying perturbations. Pac. J. Math. 277, 77-97 (2015)

103. Gaspard, P., Rice, S.A.: Semiclassical quantization of the scattering from a classically chaotic repellor. J. Chem. Phys. 90, 224-2254 (1989)

104. Gérard, C.: Asymptotique des pôles de la matrice de scattering pour deux obstacles strictement convexes. Mémoires de la Société Mathématique de France Sér. 2(31), 1-146 (1988)

105. Gérard, C., Martinez, A.: Semiclassical asymptotics for the spectral function of long range Schrödinger operators. J. Funct. Anal. 84, 226-254 (1989)

106. Gérard, C., Martinez, A., Robert, D.: Breit-Wigner formulas for the scattering phase and the total scattering cross-section in the semi-classical limit. Commun. Math. Phys. 121, 323-336 (1989)

107. Gérard, C., Sjöstrand, J.: Semiclassical resonances generated by a closed trajectory of hyperbolic type. Commun. Math. Phys. 108, 391-421 (1987)

108. Gérard, C., Sjöstrand, J.: Resonances en limite semiclassique et exposants de Lyapunov. Commun. Math. Phys. 116, 193-213 (1988)

109. Giulietti, P., Liverani, C., Pollicott, M.: Anosov flows and dynamical zeta functions. Ann. Math. 178(2), 687-773 (2013)

110. Goodhue, W.L.: Scattering theory for hyperbolic systems with coefficients of Gevrey type. Trans. Am. Math. Soc. 180, 337-346 (1973)

111. Goussev, A., Schubert, R., Waalkens, H., Wiggins, S.: Quantum theory of reactive scattering in phase space. Adv. Quant. Chem. 60, 269-332 (2010)

112. Gouëzel, S., Liverani, C.: Banach spaces adapted to Anosov systems. Ergod. Theory Dyn. Syst. 26, 189-217 (2006)

113. Gribov, V.: The Theory of Complex Angular Momentum. Cambridge University Press, Cambridge (2003)

114. Grigis, A., Martinez, A.: Resonance widths for the molecular predissociation. Anal. PDE 7, 10271055 (2014)

115. Guillarmou, C.: Meromorphic properties of the resolvent on asymptotically hyperbolic manifolds. Duke Math. J. 129, 1-37 (2005)

116. Guillarmou, C.: Invariant distributions and X-ray transform for Anosov flows. J. Diff. Geom. to appear. arXiv: 1408.4732

117. Guillarmou, C.: Lens rigidity for manifolds with hyperbolic trapped set. J. AMS, to appear arXiv: 14102.1760 
118. Guillarmou, C., Hilgert, J., Weich, T.: Classical and quantum resonances for hyperbolic surfaces. preprint, arXiv: 1605.08801

119. Guillarmou, C., Mazzeo, R.: Resolvent of the Laplacian on geometrically finite hyperbolic manifolds. Invent. Math. 187, 99-144 (2012)

120. Guillarmou, C., Monard, F.: Reconstruction formulas for X-ray transforms in negative curvature. arXiv: 1511.05516

121. Guillarmou, C., Paternain, G., Salo, M., Uhlmann, G.: The X-ray transform for connections in negative curvature. Commun. Math. Phys. 343, 83-127 (2016)

122. Guillopé, L., Lin, K.K., Zworski, M.: The Selberg zeta function for convex co-compact Schottky groups. Commun. Math. Phys. 245, 149-176 (2004)

123. Guillopé, L., Zworski, M.: Wave trace for Riemann surfaces. Geom. Funct. Anal. 6, 1156-1168 (1999)

124. Hargé, T., Lebeau, G.: Diffraction par un convexe. Invent. Math. 118, 161-196 (1994)

125. Helffer, B., Sjöstrand, J.: Resonances en limite semiclassique. Bull. Soc. Math. Fr. 114(24-25) (1986)

126. Hilgert, J., Pasquale, A., Przebinda, T.: Resonances for the Laplacian on Riemannian symmetric spaces: the case of $S L(3, R) / S O(3)$. arXiv: 1411.6527

127. Hintz, P.: Resonance expansions for tensor-valued waves on asymptotically Kerr-de Sitter spaces. arXiv: 1502.03183, to appear in J. Spectr. Theory

128. Hintz, P., Vasy, A.: Non-trapping estimates near normally hyperbolic trapping. Math. Res. Lett. 21, 1277-1304 (2014)

129. Hintz, P., Vasy, A.: Global analysis of quasilinear wave equations on asymptotically Kerr-de Sitter spaces. arXiv: 1404.1348

130. Hintz, P., Vasy, A.: Semilinear wave equations on asymptotically de Sitter, Kerr-de Sitter and Minkowski spacetimes. Anal. PDE 8, 1807-1890 (2015)

131. Hintz, P., Vasy, A.: The global non-linear stability of the Kerr-de Sitter family of black holes. arXiv: 1606.04014

132. Hirsch, M., Pugh, C., Shub, M.: Invariant Manifolds. Lecture Notes in Mathematics. Springer, Berlin (1977)

133. Hislop, P.D., Sigal, I.M.: Introduction to spectral theory: with applications of to Schrödinger operators. Appl. Math. Sci. 113, Springer (1996)

134. Holmer, J., Liu, C.: Blow-up for the 1D nonlinear Schrödinger equation with point nonlinearity I: Basic theory arXiv: 1510.03491

135. Holmer, J., Zworski, M.: Breathing patterns in nonlinear relaxation. Nonlinearity 22, 1259-1301 (2009)

136. Hörmander, L.: The Analysis of Linear Partial Differential Operators I. Distribution Theory and Fourier Analysis. Springer, Berlin (1983)

137. Hörmander, L.: The Analysis of Linear Partial Differential Operators III. Pseudo-Differential Operators. Springer, Berlin (1985)

138. Hunziker, W.: Distortion analyticity and molecular resonance curves. Ann. Inst. H. Poincaré Phys. Théor. 45, 339-358 (1986)

139. Ikawa, M.: On the poles of the scattering matrix for two strictly convex obstacles. J. Math. Kyoto Univ. 23(1), 127-194 (1983)

140. Ikawa, M.: Decay of solutions of the wave equation in the exterior of several convex bodies. Ann. Inst. Fourier 38, 113-146 (1988)

141. Ingremeau, M.: Distorted plane waves in chaotic scattering. arXiv: 1507.02970

142. Intissar, A.: A polynomial bound on the number of the scattering poles for a potential in evendimensional spaces. Commun. Partial Differ. Equ. 11, 367-396 (1986)

143. Iommi, G.: The Bowen Formula: Dimension Theory and Thermodynamic Formalism. Notes from VII Escuela de Sistemas Dinámicos, Valparaíso, (2008) http://www.mat.uc.cl/ giommi/notas3_escuela

144. Ivrii, V.: 100 year's of Weyl law. Bull. Math. Sci. (2016) http://link.springer.com/journal/13373

145. Jagau, T.-C., Zuev, D., Bravaya, K.B., Epifanovsky, E., Krylov, A.I.: A fresh look at resonances and complex absorbing potentials: density matrix-based approach. J. Phys. Chem. Lett. 5, 310-315 (2014)

146. Jakobson, D., Naud, F.: Lower bounds for resonances of infinite area Riemann surfaces. Anal. PDE 3, 207-225 (2010)

147. Jakobson, D., Naud, F.: On the critical line of convex co-compact hyperbolic surfaces. Geom. Funct. Anal. 22, 352-368 (2012)

148. Jakobson, D., Naud, F.: Resonances and convex co-compact congruence subgroups of $P S L_{2}(\mathbb{Z})$. Israel J. Math. 213, 443-473 (2016) 
149. Jensen, A., Kato, T.: Spectral properties of Schrödinger operators and time-decay of the wave functions. Duke Math. J. 46, 583-611 (1979)

150. Jensen, A., Nenciu, G.: A unified approach to resolvent expansions at thresholds, Rev. Math. Phys. 13(2001), 717-754; Erratum. Rev. Math. Phys. 16, 675-677 (2004)

151. Jin, L.: Resonance-free region in scattering by a strictly convex obstacle. Ark. Mat. 52, 257-289 (2014)

152. Jin, L.: Scattering resonances of convex obstacles for general boundary conditions. Commun. Math. Phys. 335, 759-807 (2015)

153. Jin, L., Zworski, M.: A local trace formula for Anosov flows. with an appendix by F. Naud, Ann. Henri Poincaré, to appear, arXiv: 1411.6177

154. Katok, A., Hasselblatt, B.: Introduction to the Modern Theory of Dynamical Systems. Cambridge University Press, Cambridge (1997)

155. Keller, G., Liverani, C.: Stability of the spectrum for transfer operators. Ann. Scuola Norm. Sup. Pisa Cl. Sci (4)28, 141-152 (1999)

156. Klopp, F., Zworski, M.: Generic simplicity of resonances. Helv. Phys. Acta 68, 531-538 (1995)

157. Kokkotas, K., Schmidt, B.: Quasi-normal modes of stars and black holes. Living Rev. Relativ. 2, 2 (1999)

158. Körber, M.J., Bäcker, A., Ketzmerick, R.: Localization of chaotic resonance states due to a partial transport barrier. Phys. Rev. Lett. 115, 254101 (2015)

159. Körber, M.J., Michler, M., Bäcker, A., Ketzmerick, R.: Hierarchical fractal Weyl laws for chaotic resonance states in open mixed systems. Phys. Rev. Lett. 111, 114102 (2013)

160. Korotyaev, E.: Stability for inverse resonance problem. Int. Math. Res. Notices 2004(73), 3927-3936 (2004)

161. Korotyaev, E.: Inverse resonance scattering on the real line. Inverse Probl. 21, 325 (2005)

162. Lax, P.D., Phillips, R.S.: Scattering Theory. Academic Press, Cambridge (1968)

163. Lax, P.D., Phillips, R.S.: The asymptotic distribution of lattice points in Euclidean and non-Euclidean spaces. J. Funct. Anal. 46, 280-350 (1982)

164. Lebeau, G.: Régularité Gevrey 3 pour la diffraction. Commun. Partial Differ. Equ. 9, 1437-1494 (1984)

165. Lebeau, G.: Équations de Fokker-Planck géométriques. II. Estimations hypoelliptiques maximales. Ann. Inst. Fourier 57, 1285-1314 (2007)

166. Lebeau, G., Zworski, M.: Remarks on Vasy's operator with analytic coefficients. https://math.berkeley. edu/ zworski/hypo.pdf

167. Lin, K.K.: Numerical study of quantum resonances in chaotic scattering. J. Comput. Phys. 176, 295-329 (2002)

168. Liverani, C.: On contact Anosov flows. Ann. Math. 159(2), 1275-1312 (2004)

169. Liverani, C.: Fredholm determinants, Anosov maps and Ruelle resonances. Discrete Contin. Dyn. Syst. 13, 1203-1215 (2005)

170. Lu, W., Sridhar, S., Zworski, M.: Fractal Weyl laws for chaotic open systems. Phys. Rev. Let. 91, 154101 (2003)

171. Wei, M., Majda, G., Strauss, W.: Numerical computation of the scattering frequencies for acoustic wave equations. J. Comput. Phys. 75, 345-358 (1988)

172. Mandouvalos, N.: Spectral theory and Eisenstein series for Kleinian groups. Proc. Lond. Math. Soc. 57, 209-238 (1988)

173. Marklof, J.: Selberg's trace formula: an introduction. In: Bolte, J., Steiner, F. (eds.) Hyperbolic Geometry and Applications in Quantum Chaos and Cosmology, pp. 83-119. Cambridge University Press, Cambridge (2011)

174. Martinez, A.: Resonance free domains for non globally analytic potentials. Ann. Henri Poincaré 4, 739-756 (2002)

175. Mashreghi, J., Nazarov, F., Havin, V.: Beurling-Malliavin multiplier theorem: the seventh proof. St. Petersburg Math. J. 17, 699-744 (2006)

176. Mazzeo, R.R., Melrose, R.B.: Meromorphic extension of the resolvent on complete spaces with asymptotically constant negative curvature. J. Funct. Anal. 75, 260-310 (1987)

177. Mazzeo, R.R., Vasy, A.: Analytic continuation of the resolvent of the Laplacian on symmetric spaces of noncompact type. J. Funct. Anal. 228, 311-368 (2005)

178. Mazzeo, R.R., Vasy, A.: Scattering theory on $S L(3) / S O(3)$ : connections with quantum 3-body scattering. Proc. Lond. Math. Soc. 94, 545-593 (2007) 
179. Melrose, R.B.: Singularities and energy decay in acoustical scattering. Duke Math. J. 46, 43-59 (1979)

180. Melrose, R.B.: Scattering theory and the trace formula of the wave group. J. Funct. Anal. 45, 429-440 (1982)

181. Melrose, R.B.: Growth estimates for the poles in potential scattering. unpublished manuscript (1984)

182. Melrose, R.B.: Polynomial bounds on the distribution of poles in scattering by an obstacle. Journées "Équations aux Dérivées partielles", Saint-Jean de Monts, (1984)

183. Melrose, R.B.: Spectral and scattering theory for the Laplacian on asymptotically Euclidian spaces. In: Ikawa, M. (ed.) Spectral and Scattering Theory. Marcel Dekker (1994) http://www-math.mit.edu/ $\sim$ rbm/papers/sslaes/sslaes.ps

184. Melrose, R.B.: Geometric Scattering Theory. Cambridge University Press, Cambridge (1995)

185. Merkli, M., Sigal, I.M.: A time-dependent theory of quantum resonances. Commun. Math. Phys. 201, 549-576 (1999)

186. Moon, C.R., Mattos, L.S., Foster, B.K., Zeltzer, G., Ko, W., Manoharan, H.C.: Quantum phase extraction in isospectral electronic nanostructures. Science 319, 782-787 (2008)

187. Morawetz, C.: Exponential decay of solutions of the wave equation. Commun. Pure. Appl. Math. 19, 439-444 (1966)

188. Morawetz, C.: On the modes of decay for the wave equation in the exterior of a reflecting body. Proc. R. Irish Acad. Sect. A 72, 113-120 (1972)

189. Morawetz, C., Ralston, J., Strauss, W.: Decay of solutions of the wave equation outside nontrapping obstacles. Commun. Pure. Appl. Math. 30, 447-508 (1977)

190. Moskovici, H., Stanton, R.: R-torsion and zeta functions for locally symmetric manifolds. Invent. Math. 105, 185-216 (1991)

191. Naud, F.: Expanding maps on Cantor sets and analytic continuation of zeta functions. Ann. Sci. École Norm. Sup. 38, 116-153 (2005)

192. Naud, F.: Density and location of resonances for convex co-compact hyperbolic surfaces. Invent. Math. 195, 723-750 (2014)

193. Naud, F.: Borne de Weyl fractale et résonances. Séminaire BOURBAKI, Novembre 2015 68ème année, 2015-2016, no. 1107, http://www.bourbaki.ens.fr/TEXTES/1107.pdf

194. Nakamura, S., Stefanov, P., Zworski, M.: Resonance expansions of propagators in the presence of potential barriers. J. Funct. Anal. 205, 180-205 (2003)

195. Nakano, Y., Wittsten, J.: On the spectra of a randomly perturbed partially expanding map on the torus. Nonlinearity 28, 951-1002 (2015)

196. Nonnenmacher, S.: Spectral problems in open quantum chaos. Nonlinearity 24, R123-R167 (2011)

197. Nonnenmacher, S., Sjöstrand, J., Zworski, M.: From open quantum systems to open quantum maps. Commun. Math. Phys. 304, 1-48 (2011)

198. Nonnenmacher, S., Sjöstrand, J., Zworski, M.: Fractal Weyl law for open quantum chaotic maps. Ann. Math. 179(2), 179-251 (2014)

199. Nonnenmacher, S., Zworski, M.: Distribution of resonances for open quantum maps. Commun. Math. Phys. 269, 311-365 (2007)

200. Nonnenmacher, S., Zworski, M.: Quantum decay rates in chaotic scattering. Acta Math. 203, 149-233 (2009)

201. Nonnenmacher, S., Zworski, M.: Semiclassical resolvent estimates in chaotic scattering. Appl. Math. Res. eXpress (2009). doi:10.1093/amrx/abp003

202. Nonnenmacher, S., Zworski, M.: Decay of correlations for normally hyperbolic trapping. Invent. Math. 200, 345-438 (2015)

203. Oh, H., Winter, D.: Uniform exponential mixing and resonance free regions for convex co-compact congruence subgroups of $S L_{2}(\mathbb{Z})$. J. AMS 29, 1069-1115 (2016)

204. Parry, W., Pollicott, M.: An analogue of the prime number theorem for closed orbits of Axiom A flows. Ann. Math. 118, 573-591 (1983)

205. Patterson, S.J.: The Laplacian operator on a Riemann surface I. Compos. Math. 31, 83-107 (1975)

206. Patterson, S.J.: The Laplacian operator on a Riemann surface II. Compos. Math 32, 71-112 (1976)

207. Patterson, S.J.: The Laplacian operator on a Riemann surface III. Compos. Math 33, 71-112 (1976)

208. Perelman, G.: On the formation of singularities in solutions of the critical nonlinear Schrödinger equation. Ann. H. Poincaré 2, 605-673 (2001)

209. Perry, P.A.: The Laplace operator on a hyperbolic manifold. II. Eisenstein series and the scattering matrix. J. Reine Angew. Math. 398, 67-91 (1989) 
210. Petkov, V., Stoyanov, L.: Analytic continuation of the resolvent of the Laplacian and the dynamical zeta function. Anal. PDE 3, 427-489 (2010)

211. Petkov, V., Zworski, M.: Breit-Wigner approximation and distribution of resonances, Comm. Math. Phys. 204, 329-351 (1999); Erratum. Comm. Math. Phys. 214, 733-735 (2000)

212. Petkov, V., Zworski, M.: Semi-classical estimates on the scattering determinant. Ann. H. Poincaré 2 , 675-711 (2001)

213. Pollicott, M.: Meromorphic extensions of generalized zeta functions. Invent. Math. 85, 147-164 (1986)

214. Pollicott, M.: Dynamical zeta functions. http://homepages.warwick.ac.uk/ masdbl/grenoble-16july. pdf

215. Popov, G.: Asymptotics of Green's functions in the shadow. C. R. Acad. Bulgare Sci. 38, 1287-1290 (1985)

216. Potzuweit, A., Weich, T., Barkhofen, S., Kuhl, U., Stöckmann, H.-J., Zworski, M.: Weyl asymptotics: from closed to open systems. Phys. Rev. E. 86, 066205 (2012)

217. Prony, G.R.: Essai éxperimental et analytique: sur les lois de la dilatabilité, J. École Polytechnique, Floréal et Plairial, an III 1, cahier 22, 24-76 (1795)

218. Ralston, J.: Solutions of the wave equation with localized energy. Commun. Pure Appl. Math. 22, 807-823 (1969)

219. Ralston, J.: Addendum to: "The first variation of the scattering matrix" J. Differ. Equ. 21(2), 378-394 (1976) by J. W. Helton and Ralston. J. Differ. Equ. 28, 155-162 (1978)

220. Ramond, T.: Analyse semiclassique, résonances et contrôle de l'équation de Schrödinger. on-line lecture notes (2005) http://www.math.u-psud.fr/ ramond/docs/m2/cours.pdf

221. Regge, T.: Analytic properties of the scattering matrix. Il Nuovo Cimento 8, 671-679 (1958)

222. Reinhardt, W.P.: Complex scaling in atomic and molecular physics, in and out of external fields. AMS Proc. Ser. Proc. Symp. Pure Math. 76, 357-377 (2007)

223. Riss, U.V., Meyer, H.D.: Reflection-free complex absorbing potentials. J. Phys. B 28, 1475-1493 (1995)

224. Rodnianski, I., Tao, T.: Effective limiting absorption principles and applications. Commun. Math. Phys. 333, 1-95 (2015)

225. Rouleux, M.: Absence of resonances for semiclassical Schrödinger operators with Gevrey coefficients. Hokkaido Math. J. 30, 475-517 (2001)

226. Ruelle, D.: Zeta-functions for expanding maps and Anosov flows. Invent. Math. 34, 231-242 (1976)

227. Ruelle, D.: Resonances of chaotic dynamical systems. Phys. Rev. Lett. 56, 405-407 (1986)

228. Rugh, H.H.: Generalized Fredholm determinants and Selberg zeta functions for Axiom A dynamical systems. Erg. Theory Dyn. Syst. 16, 805-819 (1996)

229. Sá Barreto, A.: Remarks on the distribution of resonances in odd dimensional Euclidean scattering. Asymptot. Anal. 27, 161-170 (2001)

230. Sá Barreto, A., Zworski, M.: Existence of resonances in potential scattering. Commun. Pure Appl. Math. 49, 1271-1280 (1996)

231. Sá Barreto, A., Zworski, M.: Distribution of resonances for spherical black holes. Math. Res. Lett. 4, 103-121 (1997)

232. Schenck, E.: Energy decay for the damped wave equation under a pressure condition. Commun. Math. Phys. 300, 375-410 (2010)

233. Schlag, W.: Dispersive estimates for Schrödinger operators: a survey. In: Mathematical aspects of nonlinear dispersive equations, pp. 255-285, Ann. of Math. Stud., 163, Princeton University Press, Princeton, NJ (2007)

234. Schubert, R., Waalkens, H., Wiggins, S.: Efficient computation of transition state resonances and reaction rates from a quantum normal form. Phys. Rev. Lett. 96, 218302 (2006)

235. Seideman, T., Miller, W.H.: Calculation of the cumulative reaction probability via a discrete variable representation with absorbing boundary conditions. J. Chem. Phys. 96, 4412-4422 (1992)

236. Shapiro, J.: Semiclassical resolvent bounds in dimension two. arXiv: 1604.03852

237. Shen, S.: Analytic torsion, dynamical zeta functions and orbital integrals. C.R. Acad. Sci. Paris Ser. I 354, 433-436 (2016)

238. Shlapentokh-Rothman, Y.: Quantitative mode stability for the wave equation on the Kerr spacetime. Ann. Inst. Henri Poincaré (A) 16, 289-345 (2015)

239. Simon, B.: The definition of molecular resonance curves by the method of exterior complex scaling. Phys. Lett. 71A, 211-214 (1979) 
240. Sjöstrand, J.: Geometric bounds on the density of resonances for semiclassical problems. Duke Math. J. 60, 1-57 (1990)

241. Sjöstrand, J.: A trace formula and review of some estimates for resonances. In: Microlocal analysis and spectral theory (Lucca, 1996), pp. 377-437, NATO Adv. Sci. Inst. Ser. C Math. Phys. Sci., 490, Kluwer Acadmic Publication, Dordrecht (1997)

242. Sjöstrand, J.: A trace formula for resonances and application to semi-classical Schrödinger operators. Séminaire Équations aux dérivées partielles (1996-1997), vol. 1996-1997, 1-13

243. Sjöstrand, J.: Lectures on resonances. version préliminaire, printemps (2002) http://sjostrand.perso. math.cnrs.fr/Coursgbg.pdf

244. Sjöstrand, J.: Weyl law for semi-classical resonances with randomly perturbed potentials. Mém. Soc. Math. Fr. (N.S.) 136, vi+144 (2014)

245. Sjöstrand, J., Zworski, M.: Complex scaling and the distribution of scattering poles. J. Am. Math. Soc. 4, 729-769 (1991)

246. Sjöstrand, J., Zworski, M.: The complex scaling method for scattering by strictly convex obstacles. Ark. Mat. 33, 135-172 (1995)

247. Sjöstrand, J., Zworski, M.: Asymptotic distribution of resonances for convex obstacles. Acta Math. 183, 191-253 (1999)

248. Sjöstrand, J., Zworski, M.: Fractal upper bounds on the density of semiclassical resonances. Duke Math. J. 137, 381-459 (2007)

249. Smale, S.: Differentiable dynamical systems. Bull. Am. Math. Soc. 73, 747-817 (1967)

250. Smith, H.: A calculus for three-dimensional CR manifolds of finite type. J. Funct. Anal. 120, 135-162 (1994)

251. Smith, H.: Parametrix for a semiclassical sum of squares. in preparation

252. Smith, H., Zworski, M.: Heat traces and existence of scattering resonances for bounded potentials. Ann. Inst. Fourier 66, 455-475 (2016)

253. Soffer, A., Weinstein, M.I.: Time dependent resonance theory. Geom. Funct. Anal. 8, 1086-1128 (1998)

254. Stefanov, P.: Quasimodes and resonances: sharp lower bounds. Duke Math. J. 99, 75-92 (1999)

255. Stefanov, P.: Sharp upper bounds on the number of the scattering poles. J. Funct. Anal. 231, 111-142 (2006)

256. Stefanov, P., Vodev, G.: Neumann resonances in linear elasticity for an arbitrary body. Commun. Math. Phys. 176, 645-659 (1996)

257. Stoyanov, L.: Spectra of Ruelle transfer operators for Axiom A flows. Nonlinearity 24, 1089-1120 (2011)

258. Tamura, H.: Aharonov-Bohm effect in resonances for scattering by three solenoids at large separation, preprint (2015)

259. Tamura, H.: Aharonov-Bohm effect in resonances for scattering by three solenoids. Proc. Jpn. Acad. Ser. A Math. Sci 91(2015), 45-49 (2015)

260. Tang, S.H., Zworski, M.: From quasimodes to resonances. Math. Res. Lett. 5, 261-272 (1998)

261. Tsujii, M.: Contact Anosov flows and the FBI transform. Ergod. Theory Dyn. Syst. 32, 2083-2118 (2012)

262. Vainberg, B.R.: Exterior elliptic problems that depend polynomially on the spectral parameter and the asymptotic behavior for large values of the time of the solutions of nonstationary problems. (Russian) Mat. Sb. (N.S.) 92(134), 224-241 (1973)

263. Vasy, A.: Microlocal analysis of asymptotically hyperbolic and Kerr-de Sitter spaces, with an appendix by Semyon Dyatlov. Invent. Math. 194, 381-513 (2013)

264. Vasy, A.: Microlocal analysis of asymptotically hyperbolic spaces and high energy resolvent estimates. Inverse problems and applications. Inside Out II, edited by Gunther Uhlmann, Cambridge University Press, MSRI publications 60 (2012)

265. Vodev, G.: Sharp bounds on the number of scattering poles for perturbations of the Laplacian. Commun. Math. Phys. 146, 205-216 (1992)

266. Vodev, G.: Sharp bounds on the number of scattering poles in even-dimensional spaces. Duke Math. J. 74, 1-17 (1994)

267. Vodev, G.: Sharp bounds on the number of scattering poles in the two dimensional case. Math. Nachr. 170, 287-297 (1994)

268. Vodev, G.: Semi-classical resolvent estimates and regions free of resonances. Math. Nachr. 287, 825-835 (2014) 
269. Warnick, C.: On quasinormal modes of asymptotically Anti-de Sitter black holes. Commun. Math. Phys. 333, 959-1035 (2015)

270. Weich, T.: Resonance chains and geometric limits on Schottky surfaces. Commun. Math. Phys. 337, 727-765 (2015)

271. Weich, T.: On the support of Pollicott-Ruelle resonanant states for Anosov flows. Ann. Henri Poincaré, to appear, arXiv: 1511.08338

272. Wunsch, J.: Diffractive propagation on conic manifolds. Séminaire Laurent Schwartz, (2016). http:// slsedp.cedram.org/slsedp-bin/fitem?id=SLSEDP_2015-2016 A9_0

273. Wunsch, J., Zworski, M.: Distribution of resonances for asymptotically euclidean manifolds. J. Differ. Geom. 55, 43-82 (2000)

274. Wunsch, J., Zworski, M.: Resolvent estimates for normally hyperbolic trapped sets. Ann. Inst. Henri Poincaré (A) 12, 1349-1385 (2011)

275. Zuily, C.: Real analyticity of radiation patterns on asymptotically hyperbolic manifolds. (2017) arXiv: 1611.10250, to appear in Applied Mathematics Research eXpress

276. Zworski, M.: Distribution of poles for scattering on the real line. J. Funct. Anal. 73, 277-296 (1987)

277. Zworski, M.: Sharp polynomial bounds on the number of scattering poles of radial potentials. J. Funct. Anal. 82, 370-403 (1989)

278. Zworski, M.: Sharp polynomial bounds on the number of scattering poles. Duke Math. J. 59, 311-323 (1989)

279. Zworski, M.: Counting scattering poles. In: Ikawa, M. (ed.) Spectral and Scattering Theory, Lecture Notes in Pure and Applied Mathematics. Marcel Dekker, New York (1994)

280. Zworski, M.: Dimension of the limit set and the density of resonances for convex co-compact hyperbolic surfaces. Invent. Math. 136, 353-409 (1999)

281. Zworski, M.: Semiclassical Analysis. Graduate Studies in Mathematics, vol. 138. American Mathematical Society, Providence (2012)

282. Zworski, M.: Resonances for asymptotically hyperbolic manifolds: Vasy's method revisited. arXiv: 1511.03352, to appear in J. Spectr. Theory

283. Zworski, M.: Scattering resonances as viscosity limits. arXiv: 1505.00721, to appear in "Algebraic and Analytic Microlocal Analysis”, Hitrik, M., Tamarkin, D., Tsygan B., Zelditch, S. (eds.), Springer 